\title{
Teoria de poda na família de Hénon
}

\author{
Juan Valentín Mendoza Mogollón \\ TESE APRESENTADA \\ $\mathrm{AO}$ \\ Instituto De Matemática e Estatística \\ DA \\ Universidade DE SÃO PAUlo \\ PARA \\ OBTENÇÃO DO TÍTULO \\ $\mathrm{DE}$ \\ Doutor EM CIÊNCIAS
}

Programa: Matemática Aplicada

Orientador: Prof. Dr. André Salles de Carvalho

Durante o desenvolvimento deste trabalho o autor recebeu auxílio financeiro da FAPESP Processo N 2007/55771-0

São Paulo, Fevereiro de 2011 


\title{
Teoria de poda na família de Hénon
}

\author{
Este exemplar corresponde à redação \\ final da tese devidamente corrigida \\ e defendida por Juan Valentín Mendoza Mogollón \\ e aprovada pela Comissão Julgadora.
}

Banca Examinadora:

- Prof. Dr. André Salles de Carvalho (orientador) - IME-USP.

- Prof. Dr. Fabio A. Tal - IME-USP.

- Prof. Dr. Isabel Rios - UFF.

- Prof. Dr. Philip Boyland - UFL.

- Prof. Dr. Daniel Smania - ICMC-USP. 
Dedico este trabalho aos meus pais: Inelda e Valentín. 


\section{Agradecimentos}

Eu gostaria agradecer a cada uma das pessoas que, de diferentes maneiras, me ajudaram a concluir este trabalho. Especialmente, gostaria agradecer a meu orientador André de Carvalho por introduzir-me na pesquisa relacionada à teoria de poda, compartilhando comigo as suas ideias, que deram origem a esta tese, e pelo seu apoio decidido em cada momento que duraram os meus estudos de doutorado. A minha dívida com ele é tão grande quanto o meu agradecimento.

A Jorge e Elizangela Avila, e Juan Carlos e Fatima Aguilar por me receber como um irmão aqui em São Paulo.

A Dra. Helena de Castro que me forneceu todas as facilidades para fazer o Curso de Verão 2007. Aos professores do IME: Salvador Zanata, Jorge Sotomayor, Eduardo Colli pelas ensinamentos e estímulo que me passaram. Ao grupo de Sistemas Dinâmicos do IME pelas interessantes palestras que, religiosamente, cada sexta feira organizam. Ao Toby Hall quem me enviou o programa para desenhar variedades invariantes na família de Hénon.

A meus amigos no IME: Braulio García, Juliano Gonchorowski, Taty Batista, Graciele Pereira, Sebastian Vidal, Priscila Barbosa, Peter Hazard e muitos outros aos quais peço desculpas por não escrever seu nome aqui. Muito obrigado pelo companheirismo e pelas tantas manhãs e tardes de conversa e de café.

Aos funcionarios do IME, da Biblioteca, da secretaria da CPG e do Departamento de Matemática Aplicada por serem sempre amáveis comigo.

A meus amigos de Piura: Ellis Hidalgo, Segundo Correa, Carlos Imán, Jenniel Ruiz, Tania Heredia e, em especial a Ricardo Velesmoro pelos seus incentivos para estudar doutorado em Brasil e pela sua ajuda incondicional.

À Fapesp, pelo apoio financeiro e por me brindar a oportunidade de fazer o doutorado direto.

Finalmente, eu gostaria agradecer a minha família pela sua compreensão e paciencia e por entenderem que, apesar dos miles de quilômetros que nos separam, sempre estão no meu coração. 


\section{Resumo}

A teoria de poda é um caminho para dar uma descrição topologica de famílias de homeomorfismos de superfície. Nesta tese desenvolvemos uma teoria de poda diferenciável. Primeiro definimos discos de poda para o exemplo paradigmático da ferradura de Smale e provamos um teorema de poda diferenciável. Depois, com uma construção similar a derivados de Anosov, extendemos este teorema para difeomorfismos hiperbólicos.

Também aplicamos estas construções ao estudo da família de Hénon real e mostramos como se relaciona esta teoria com a família de Hénon complexa. Assim, provamos a Conjectura da Frente de Poda para alguns parâmetros reais na família de transformações de Hénon.

Palavras-chave: teoria de poda, família de Hénon, dinâmica simbólica. 


\section{Abstract}

Pruning is originally a way of giving a topological description of the dynamics of families of surface homeomorphisms. A differentiable pruning theory is developed here. First pruning discs and the pruning theorem are presented for Smale's horseshoe, which is the paradigmatic chaotic dynamical system in dimension 2. Then this is generalized to hyperbolic surface diffeomorphisms.

This is then combined with complex and numerical techniques to give a computer assisted proof of the Pruning Front Conjecture for certain open sets of (real) parameters in the Hénon family.

Keywords: Pruning, Hénon family, symbolic dynamics. 


\section{Sumário}

Lista de Figuras vi

1 Introdução 1

1.1 A teoria de poda . . . . . . . . . . . . . . 2

1.2 Definição de problema . . . . . . . . . . . . . . . 4

1.3 Contribuições ..................... 4

2 Conceitos $\quad 8$

2.1 Preliminares.................... 8

2.1.1 Terminologia e notações . . . . . . . . . . . . . 8

2.1.2 Subshifts de tipo finito . . . . . . . . . . . . . . 9

2.1.3 A ferradura de Smale .............. . 9

2.1.4 Plano simbólico . . . . . . . . . . . . . . . . 11

2.1.5 Hiperbolicidade . . . . . . . . . . . . . . . 12

2.1.6 Partições de Markov . . . . . . . . . . . . . . . . . . 13

2.2 Propriedades de transformações de Hénon . . . . . . . . . . . . . 14

2.2.1 A ferraduras na família de Hénon . . . . . . . . . . . . . . 16

2.3 Teoria da Frente de Poda . . . . . . . . . . . . . . . 18

2.3.1 Evidência para a CFP . . . . . . . . . . . 18

2.3.2 Teoria de poda ................. 19

3 Teoria de poda na ferradura $\quad 23$

3.1 Construção do difeomorfismo de poda . . . . . . . . . . . . 23

3.1.1 Frentes de poda e discos de poda homoclínicos . . . . . 23

3.1.2 Exemplos de discos homoclínicos . . . . . . . . . . 25

3.1.3 Construção da isotopia de poda . . . . . . . . . . . . . 27

3.1.4 Construção de uma isotopia $C^{\infty}$. . . . . . . . . . . . 31

3.2 Shifts de Markov para o difeomorfismo de poda $H_{1}$. . . . . . . . 32

3.2.1 Partição de geração 1 . . . . . . . . . . . . . . 33 
3.2.2 Operações sobre os retângulos . . . . . . . . . . . . . 35

3.2.3 Algoritmo . . . . . . . . . . . . . . . 35

3.3 Prova do teorema de poda . . . . . . . . . . . . 39

4 Poda para o derivado da ferradura $\quad 41$

4.1 Derivados da ferradura . . . . . . . . . . . . . . . 41

4.1.1 Derivados de Anosov . . . . . . . . . . . . . . . 41

4.1.2 Cirugia num ponto periódico . . . . . . . . . . . . 42

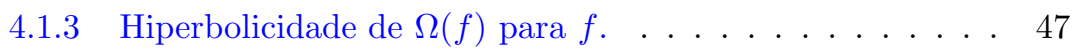

4.1.4 Relação entre $\Omega(f)$ e $\Sigma_{2}$. . . . . . . . . . . . . . . . . . 49

4.2 O teorema de poda para o derivado da ferradura $f$. . . . . . 52

4.2.1 Tipo periódico-homoclínico . . . . . . . . . . 53

4.2.2 Tipo periódico-periódico . . . . . . . . . . . . . 54

4.2.3 O Teorema de Poda para o derivado da ferradura . . . . . 56

4.3 Teoria de poda em superfícies . . . . . . . . . . . . 58

5 Frentes de poda na família de Hénon $\quad 61$

5.1 Perturbações de transformações de Hénon . . . . . . . . . . . . . 61

5.2 Frentes de poda na família de Hénon complexa . . . . . . . . . . 62

5.2.1 O lugar de ferraduras . . . . . . . . . . . 63

5.2.2 Frentes de poda e curvas em $\mathcal{H}_{0}^{\mathbb{C}}$. . . . . . . . . . . . . . 64

6 Problemas de pesquisa $\quad 68$

6.1 Quando $H_{a, b}$ preserva área . . . . . . . . . . . . . . 68

6.2 Evidência numérica . . . . . . . . . . . . . . . . 70

6.2.1 Usando um disco de poda . . . . . . . . . . . . 71

6.2.2 Usando dois discos de poda . . . . . . . . . . . . 72

6.2.3 Mais evidência numérica . . . . . . . . . . . . . . . 74

6.3 Observações . . . . . . . . . . . . . . . . . 77

6.4 Conjectura da Frente de poda no lugar de Ferraduras . . . . . . 81

A O metodo de Biham-Wenzel $\quad 83$

$\begin{array}{ll}\text { Referências Bibliográficas } & 85\end{array}$ 


\section{Lista de Figuras}

1.1 Variedades estáveis e instáveis (Figura 1.1(a)) de $H_{4.75,0.75}$, que é uma ferradura completa e o plano simbólico (Figura 1.1(b)) . . . . . . . . . 2

1.2 Variedades estáveis e instáveis (Figura 1.2(a)) de $H_{5.4,1}$, que parece ser uma ferradura incompleta e frente de poda no plano simbólico (Figura $1.2(\mathrm{~b})$ ). . . . . . . . . . . . . . . . . 3

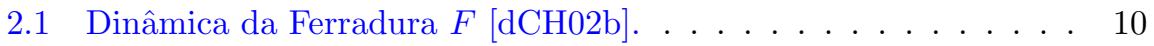

2.2 Dinâmica de uma transformação de Hénon. . . . . . . . . . . . . 15

2.3 Variedades invariantes de uma transformação de Hénon que é

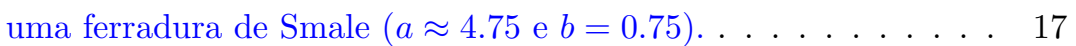

2.4 Variedades invariantes de uma transformação de Hénon com entropia maximal $(a \approx 3.3715$ e $b=0.5)$. . . . . . . . . . . . . 18

2.5 Zoom do retângulo na figura 2.4 que indica a regiâo onde está a tangência $(a \approx 3.3715$ e $b=0.5)$. . . . . . . . . . . . . . 19

2.6 Variedades estáveis e instáveis (Figura 2.6(a)) de $H_{1.9,0.05}$ e o plano simbólico para órbitas de períodos menores que 15 (Figura 2.6(b)). . . . . . . . . . . . . . . . . . 20

2.7 Relação $D_{1} \succ D_{2}$. . . . . . . . . . . . . . . . . . . . . 21

3.1 Disco homoclínico $D$ definido pelos pontos homoclínicos $p_{0}=$ $0^{\infty} 1111 \cdot 10110^{\infty}$ e $p_{1}=0^{\infty} 1110 \cdot 10110^{\infty}$. Também temos desenhado os principais elementos de um disco de poda. Os discos $D$ e $H^{-1}(D)$ são livres. . . . . . . . . . . . . . . . . . . . . . . . 25

3.2 Exemplos de discos homoclínicos . . . . . . . . . . . . 26

3.3 Funções $g_{1}, g_{2}, g_{3}$ e $g_{4}$ em um disco de poda homoclínico. . . . . 28

3.4 Partição de 1-geração para o disco de poda da figura 3.1. . . . . 34

3.5 Descrições pictóricas das operação $P_{1}$ (Ver figura 3.5(a)) e $P_{2}$ (Ver figura 3.5(b)). . . . . . . . . . . . . . . 36 
3.6 Partição de 2-geração para o disco de poda da figura 3.1. . . . . 38

3.7 Partição de 3-geração para o disco de poda da figura 3.1. . . . . 38

3.8 Partição de 4-geração para o disco de poda da figura $3.1 . \quad$. . . . 39

4.1 Ponto fixo do difeomorfismo de Anosov $f$ (Figura 6.2(a)) e o derivado de Anosov (Figura 6.2(b)) . . . . . . . . . . . . . . 42

4.2 Discos do tipo periódico homoclínico. A figura 4.2(a) corresponde a uma região livre e a figura 4.2(b) é quando $L$ é atratora. . . . . . . . 54

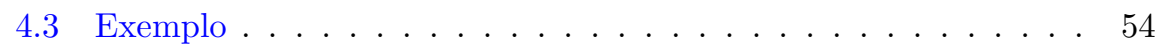

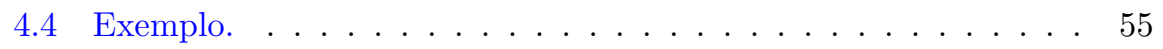

4.5 Discos do tipo periódico-periódico. . . . . . . . . . . 56

4.6 Discos do tipo periódico periódico-periódico. . . . . . . . 56

4.7 Disco de poda. . . . . . . . . . . . . . . . . 58

5.1 Partições de Markov para os valores no conjunto $A_{1}$ (figura 5.1(a)) e para os valores no conjunto $A_{2}$ (figura 5.1(b)) do teorema 5.2.4. 67

6.1 Partições de Markov conjecturadas para as transformações de Hénon $H_{3.5197,0.648}$ (figura 6.1(a)) e $H_{5.65,1}$ (figura 6.1(b)). . . . . 72

6.2 Partições de Markov conjecturadas para $H_{5.6917,1}$ (figura 6.2(a)) e para $H_{5.59,1}$ (figura $6.2(\mathrm{~b})$ ). . . . . . . . . . . . 73

6.3 Partição de Markov usando os discos $D_{3,4}$ e $D_{4,3}$ conjecturada para $H_{5.683,1} \ldots \ldots \ldots \ldots \ldots \ldots \ldots \ldots \ldots \ldots$

6.4 Partições de Markov conjecturadas para $H_{3.5,0.55}$ (figura 6.4(a)) e para $H_{2.766,0.4}$ (figura $\left.6.4(\mathrm{~b})\right) \ldots \ldots \ldots \ldots \ldots \ldots$

6.5 Partições de Markov conjecturadas para $H_{2.887,0.4}$ (figura 6.5(a)) e para $H_{2.345,0.19}$ (figura $\left.6.5(\mathrm{~b})\right) \ldots \ldots \ldots \ldots \ldots$

6.6 Partição de Markov conjecturada para $H_{2.812,0.4} \ldots \ldots \ldots \ldots$

$6.7 \quad(a \approx 2.8187+0.0119 i$ e $b=0.4) . \ldots \ldots \ldots \ldots \ldots$

$6.8(a \approx 2.8255-0.032 i$ e $b=0.4) \ldots \ldots \ldots \ldots \ldots \ldots$

$6.9 \quad(a \approx 2.8187-0.079 i$ e $b=0.4) \ldots \ldots \ldots \ldots \ldots \ldots$

$6.10(a \approx 2.8187-0.095 i$ e $b=0.4) \ldots \ldots \ldots \ldots \ldots \ldots \ldots$

$6.11(a \approx 2.8931-0.0959 i$ e $b=0.4) . \ldots \ldots \ldots \ldots \ldots$

$6.12(a \approx 3.00-0.0919 i$ e $b=0.4) \ldots \ldots \ldots \ldots \ldots$

$6.13(a \approx 3.1432-0.0919 i$ e $b=0.4) . \ldots \ldots \ldots \ldots \ldots$

$6.14(a \approx 3.1432-0.048 i$ e $b=0.4) \ldots \ldots \ldots \ldots \ldots$

$6.15(a \approx 3.149-0.00399 i$ e $b=0.4) . \ldots \ldots \ldots \ldots \ldots$ 


\section{Capítulo 1}

\section{Introdução}

O estudo da família de transformações de Hénon, introduzida por Michel Hénon em [Hén76], possui um papel paradigmático na compreensão da dinâmica de difeomorfismos no plano uma vez que aparece, com frequência, em fenômenos físicos e matemáticos. A menos de mudanças de coordenadas ${ }^{1}$, ela é dada pela fórmula:

$$
H_{a, b}(x, y):=\left(a-x^{2}-b y, x\right), a, b \in \mathbb{R}
$$

Hoje é sabido que esta família tem muitos comportamentos interessantes como a existência, em alguns parâmetros, de atratores não triviais. Isto foi demonstrado por Benedicks e Carlesson em [BC91]. Eles construiram ${ }^{2}$, para valores de $b$ muito pequenos, um conjunto $E(b)$ de valores de $a$ para os quais $H_{a, b}$ possui um atrator estranho, isto é, um atrator transitivo com expoente de Lyapunov positivo que não se reduz a uma órbita periódica.

Para outros parâmetros, a família de Hénon exibe comportamento hiperbólico o que foi demonstrado por Devaney e Nitecki em [DN79]. Eles provaram que se $a \gg 0$ então $H_{a, b}$ é uma ferradura de Smale [Sma67], isto é, $H_{a, b}$ restrito ao seu conjunto não errante $\Omega\left(H_{a, b}\right)$ é topologicamente conjugado ao shift $\sigma$ definido no espaço de sequências simbólicas $\Sigma_{2}=\{0,1\}^{\mathbb{Z}}$; também demonstraram que se $a \ll 0$ então $H_{a, b}$ têm dinâmica trivial, isto é, $\Omega\left(H_{a, b}\right)=\emptyset$.

Por um resultado de Newhouse [New79], existem parâmetros nos quais $H_{a, b}$ tem tangências homoclínicas persistentes. Isto significa que existem parâmetros $(a, b)$ tais que $H_{a, b}$ têm uma tangência homoclínica não degenerada e todo difeomorfismo $C^{r}$-próximo de $H_{a, b}$ possui também tangências homoclínicas.

Mas, existem questões que não são bem compreendidas sobre esta família. Considerando o fato que os anteriores comportamentos dinâmicos podem ocorrer, não é sabido se estes são os que prevalecem no espaço de parâmetros. Existem também interrogações abertas sobre como varia a complexidade da dinâmica quando os parâmetros são variados. Quando $a \ll 0$, a dinâmica é trivial, e quando $a \gg 0, H_{a, b}$ é uma ferradura de Smale que é um modelo de caoticidade. O que acontece para valores intermediários? Como o caos é criado?

\footnotetext{
${ }^{1}$ M. Hénon estudou a família $T_{A, B}(x, y):=\left(1-A x^{2}+y, B x\right)$, mas $T_{A, B}$ e $H_{a, b}$ são conjugadas por uma transformação linear. Ele encontrou comportamentos interessantes nos valores $A=1.4, B=0.3$, onde a transformação reverte orientação.

${ }^{2}$ Benedicks e Carlesson utilizaram também $T_{A, B}(x, y)$.
} 
Essas são algumas questões que André de Carvalho e Toby Hall abordaram em [dCH02b] e propuseram um programa para entender a dinâmica de uma transformação de Hénon mediante a teoria de poda.

\subsection{A teoria de poda}

A teoria de poda tem seus inicios no trabalho de P. Cvitanović [Cvi91] e nos estudos numéricos efetuados em [CGP88]. Nestes artigos, trata-se de entender a dinâmica de uma transformação de Hénon como uma ferradura incompleta. Mais precisamente, a idéia é a seguinte: suponha que uma transformação de Hénon é uma ferradura completa, por exemplo, uma transformação como na figura 1.1(a) onde os parâmetros são $(a, b)=(4.75,0.75)$. Cada ponto do conjunto não errante de $H_{a, b}$ tem uma representação simbólica em $\Sigma_{2}$. Então podemos construir o plano simbólico que é uma representação de todas as órbitas da ferradura, ou seja, de $\Sigma_{2}$, no quadrado $[0,1] \times[0,1]$, obtida colapsando a dinâmica irrelevante que está entre as variedades invariantes e deixando só as órbitas do conjunto não errante. Veja a figura 1.1(b). Assim, cada ponto do plano simbólico corresponde a um, dois ou a quatro pontos no conjunto não errante e tem uma representação simbólica bi-infinita.

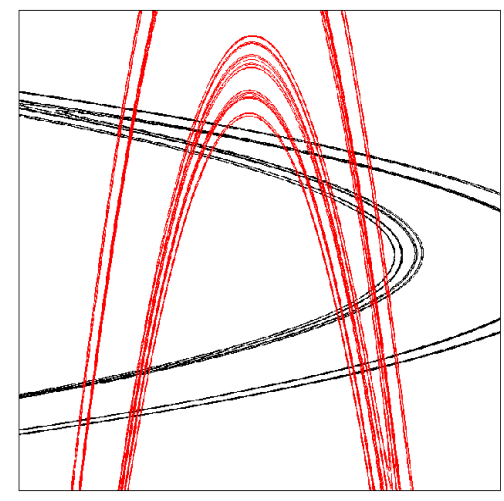

(a) Variedades estáveis e instáveis.

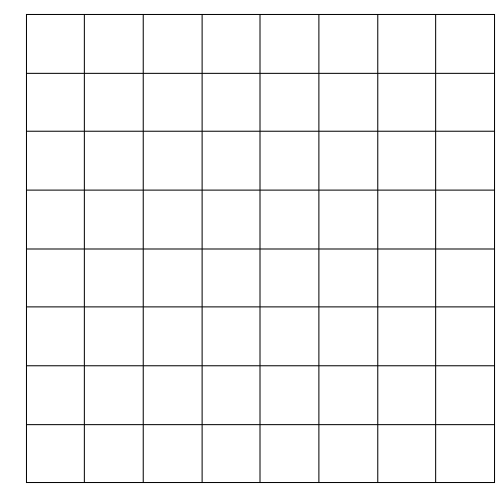

(b) Plano simbólico.

Figura 1.1: Variedades estáveis e instáveis (Figura 1.1(a)) de $H_{4.75,0.75}$, que é uma ferradura completa e o plano simbólico (Figura 1.1(b)).

Quando os parâmetros são modificados, a ferradura completa é destruida e um certo conjunto de pontos no plano simbólico pode perder as correspondentes órbitas na dinâmica da transformação. Na figura 1.2(a) podemos ver isto tomando parâmetros $(a, b)=(5.4,1)$. Note que nesta figura existem regiões onde as variedades invariantes da ferradura foram "descruzadas". Se representarmos estas órbitas no plano simbólico, como na figura 1.2(b), vamos ver regiões nas quais as órbitas foram eliminadas. Assim algumas regiões do plano simbólico são podadas e são chamadas a frente de poda.

A discussão anterior foi a motivação para que Cvitanović enunciara a Conjectura da Frente de Poda (CFP). Grosso modo esta conjectura afirma 


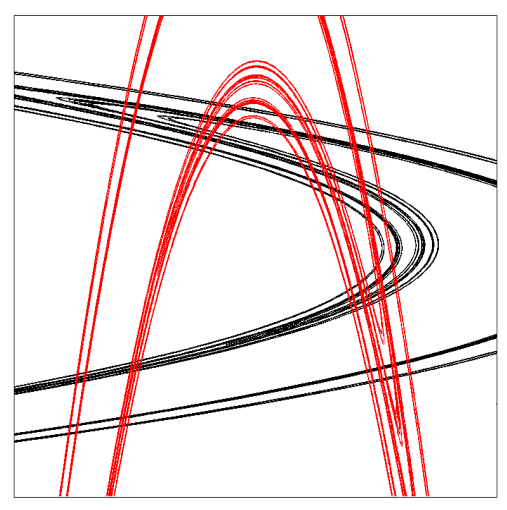

(a) Variedades estáveis e instáveis.

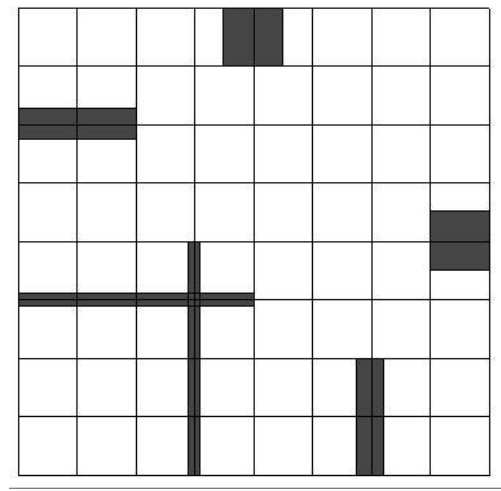

(b) Plano simbólico

Figura 1.2: Variedades estáveis e instáveis (Figura 1.2(a)) de $H_{5.4,1}$, que parece ser uma ferradura incompleta e frente de poda no plano simbólico (Figura 1.2(b)).

que cada transformação de Hénon pode ser compreendida como uma ferradura incompleta. Isto é, afirma que o conjunto não errante de toda transformação de Hénon tem uma representação no plano simbólico, a qual é obtida podando ou eliminando certas regiões dele. O resultado desta eliminação deve ser um subconjunto próprio da ferradura. Logo, o problema de entender a dinâmica de uma transformação de Hénon se reduziria então a identificar as regiões que são podadas.

Uma formulação matematicamente rigorosa da teoria de poda foi desenvolvida por A. de Carvalho em [dC99] utilizando os chamados discos de poda e deformações por isotopias. Um disco de poda para um homeomorfismo $f$ vem a ser um disco topológico $D$, satisfazendo certas propriedades dinâmicas, que contém uma região a ser podada. Neste contexto uma poda é uma isotopia que elimina dinâmica no disco de poda, isto é, uma isotopia que transforma a dinâmica não-errante de $D$ em errante. O enunciado matemático preciso é o teorema de poda:

Teorema 1.1.1 (de Carvalho). Dado um disco de poda D para um homeomorfismo $f_{0}$, existe uma isotopia $f_{t}: f_{0} \simeq f_{1}$, suportada na saturação de $D$ sob $f_{0}$ (isto é, na união de $D$ com todos os seus iterados passados e futuros sob $f_{0}$ ), tal que todos os pontos em $D$ são errantes para $f_{1}$.

Em palavras, o teorema diz que podemos destruir dinâmica num disco de poda mediante uma isotopia chamada isotopia de poda. O homeomorfismo $f_{1}$ ao final da isotopia é chamado uma poda de $f_{0}$, ou homeomorfismo de poda, e coincide com $f_{0}$ fora da saturação de $D$.

A relação entre a teoria de poda e a família de Hénon é a Conjectura da Frente de Poda de Cvitanović introduzida por Cvitanović (veja [Cvi91] para a formulação original e [dCH02b] para a formulação apresentada aqui). Definimos a família de poda $\mathcal{P}$ como o fecho (na topologia $C^{0}$ ) da família de todos os 
homeomorfismos de poda obtidos a partir da ferradura de Smale, isto é, o fecho do conjunto de todas as podas da ferradura de Smale $F=f_{0}$ obtidas usando o Teorema 1.1.1:

Conjectura da Frente de Poda (CFP). A menos de semiconjugação, a família de Hénon real está contida na família de poda, isto é, para qualquer escolha de parâmetros $a, b$ reais, a transformação de Hénon $H_{a, b}$ pertence a $\mathcal{P}$, a menos de uma semi-conjugação.

Veja o capítulo 2 para uma definição mais precisa. A conjectura afirma que explicar a dinâmica de qualquer elemento da família de Hénon é equivalente a encontrar discos de poda na ferradura de Smale $F$.

\subsection{Definição de problema}

Embora poda tenha tido êxito explicando fenômenos da dinâmica topológica de homeomorfismos do plano e da teoria dinâmica de tranças, ( [dCH02b, dCH01, dCH02a, dCH10]), a CFP continua em aberto. Em particular, só é possível descrever topologica, ou mesmo combinatoriamente, um subconjunto relativamente pequeno de todas as transformações de Hénon.

Também, identificamos dois problemas ao tentar utilizar a teorema de poda para provar a CFP. O primeiro é que, assim possamos encontrar uma quantidade considerável de discos de poda, é dificil provar que as podas associadas a eles estão na família de Hénon porque estas podas são homeomorfismos em quanto que a família de Hénon é uma família de transformações analíticas. O segundo é que o teorema de poda precisa da construção de infinitas isotopias o que pode ocasionar que a poda resultante seja um homeomorfismo não diferenciável e muito difícil de descrever, o qual elimina a possibilidade de comparar-lhe com um elemento da família de Hénon.

Nesta tese estudamos as seguintes questões:

- É possível construir transformações de poda diferenciáveis?.

- É possível construir transformações de poda hiperbólicas?

- Nos casos anteriores, qual é a dinâmica do conjunto não errante da poda?

\subsection{Contribuições}

Na primeira parte desta tese começamos o estudo da teoria de poda do ponto de vista diferenciável e na segunta parte estudamos as suas aplicações na família de Hénon.

No capítulo 2 apresentamos os conceitos a serem utilizados nos capítulos restantes como a definição de ferradura, hiperbolicidade, propriedades de transformações de Hénon, teoria de poda, entre outros.

No capítulo 3 construimos difeomorfismos de poda hiperbólicos para o caso da ferradura de Smale utilizando discos de poda homoclínicos.

Primeiro consideramos uma transformação de Hénon $H:=H_{a, b}$ que seja uma ferradura hiperbólica (veja [DN79]). Então sabemos que existe um homeo-

morfismo $h$ que conjuga a dinâmica de $H_{a, b}$, restrita a seu conjunto não errante $\Omega(H)$, e o shift $\sigma$ definido em $\Sigma_{2}=\{0,1\}^{\mathbb{Z}}$. 
Os discos de poda homoclínicos que utilizamos são definidos com órbitas homoclínicas ao ponto fixo correspondente à sequência simbólica $0^{\infty}$ e segmentos de variedades estáveis e instáveis que passam por estas órbitas homoclínicas. Para que um disco $D$ seja um disco de poda é necessario que satisfaça as condições de poda. Estas condições são ligadas à existência de uma região livre $L$ no disco $D$ que é uma região errante pela transformação $H$ e para a qual todos os seus iterados não intersectam o disco $D$. Isto faz que a caracterização de um disco de poda seja mais simples comparada com a de [dC99]. Provamos que podemos construir uma difeotopia $H_{t}$, com $H_{0}=H$, tal que o difeomorfismo $H_{1}$, ao final da isotopia, satisfaz as propriedades de poda dadas em [dC99]. $H_{1}$ é chamado difeomorfismo de poda. Mostramos também que sob estas condições é possível associar um subshift de tipo finito à dinâmica do conjunto não errante de $H_{1}$. O resultado principal é o teorema de poda seguinte:

Teorema 1.3.1. Seja $D$ um disco de poda então existe uma difeotopia $H: \mathbb{R}^{2} \times$ $[0,1] \rightarrow \mathbb{R}^{2}, \operatorname{Supp}(H) \subset D$ tal que $H_{0}=H$ e $H_{1}$ é um difeomorfismo de poda de $H$ no seguinte sentido:

(i) $\Omega\left(H_{1}\right)=\Omega(H) \backslash \cup_{i \in \mathbb{Z}} H^{i}(D)$,

(ii) $\left.H_{1}\right|_{\Omega\left(H_{1}\right)}$ é hiperbólico, e

(iii) existe um subshift de tipo finito $\left(\Sigma_{A}, \sigma_{A}\right)$ e um homeomorfismo

$$
\tau: \Omega\left(H_{1}\right) \rightarrow \Sigma_{A}
$$

tais que: $\tau \circ H_{1}=\sigma_{A} \circ \tau$.

Cabe ressaltar que a partição de Markov associada ao subshift de tipo finito do teorema anterior é obtida por um algoritmo que termina em tempo finito e do qual forneceremos detalhes na seção 3.2.

No capítulo 4 extendemos as construções do capítulo 3 a outros pontos periódicos da ferradura, isto é, construimos discos de poda com órbitas homoclínicas a pontos periódicos diferentes de $0^{\infty}$. Para isto, no ponto periódico fazemos uma deformação, similar àquela feita para derivados de Anosov, para obter um difeomorfismo chamado derivado da ferradura. Este difeomorfismo tem uma dinâmica semelhante à da ferradura exceto que o ponto periódico bifurcou em três novos pontos: dois hiperbólicos e um atrator. Logo, construir transformações de poda para o derivado da ferradura é equivalente a encontrar regiões livres ou regiões atratoras $L$ associadas ao ponto atrator e seguir as ideias do capítulo 3. Estas mesmas construções de derivados podem ser feitas para certos difeomorfismos hiperbólicos em superfícies. O seguinte teorema é uma versão diferenciável do teorema de poda de [dC99].

Teorema 1.3.2. Seja $f_{0}$ um difeomorfismo hiperbólico de uma superfície compacta $X$ e seja $D$ um disco de poda para $f_{0}$. Então existe uma difeotopia $f_{t}: f_{0} \simeq f_{1}$ tal que $f_{1}$ é hiperbólico e $\Omega\left(f_{1}\right)=\Omega(f) \backslash \cup_{i \in \mathbb{Z}} f^{k}(D)$. 
O feito nesta primeira parte fornece uma maquinaria para construir a família de poda diferenciável de um difeomorfismo $f$ : dado um disco $D_{0}$, se for necessário construimos o derivado de $f$, podamos o conjunto não errante em $D_{0}$. Se a poda $f_{1}$ tem um disco de poda $D_{1}$, podemos podar novamente para obter o difeo de poda $f_{2}$, e assim sucessivamente. A família de poda diferenciável é a coleção de todos os difeos obtidos pela aplicação deste processo repetidas vezes.

A segunda parte da tese é o capítulo 5 . Neste discutimos as relações entre a teoria de poda e a dinâmica na família de Hénon. As transformações de Hénon que estão na fronteira dos parâmetros hiperbólicos que correspondem a ferraduras completas são transformações de Hénon não hiperbólicas mas que têm entropia maximal (Veja os preliminares no capítulo 2 para esta definição). Estas transformações estão em parâmetros nos quais ocorre a primeira bifurcação. Provamos que $C^{0}$-próximos destas transformações existem transformações de poda. O enunciado formal do resultado é o seguinte teorema.

Teorema 1.3.3. Seja $H_{a_{0}, b_{0}}$ uma transformação de Hénon com entropia maximal que não é hiperbólica. Então, existe um $C^{1}$-aberto $\mathcal{U} \neq \emptyset$ em $\operatorname{Diff}^{1}\left(\mathbb{R}^{2}\right)$ tal que:

(a) dado $\epsilon>0$, existe $f_{\epsilon} \in \mathcal{U}$ tal que $d_{C^{0}}\left(H_{a_{0}, b_{0}}, f_{\epsilon}\right)<\epsilon$,

(b) se $f \in \mathcal{U}$ então $\Omega(f)$ é hiperbólico e existe um disco de poda $D_{f}$ tal que fé topologicamente conjugado ao difeomorfismo de poda $H_{D_{f}}$ associado a $D_{f}$ como no teorema 1.3.1 quando ambos estão restritos aos seus conjuntos não errantes.

No capítulo 5 também estudamos as conexões entre a teoria de poda e a dinâmica da transformação de Hénon complexa. Estas conexões estão relacionadas com o lugar de ferraduras que é o conjunto de todos os parâmetros complexos tais que a transformação de Hénon é uma ferradura complexa. Para isto, dada uma transformação de poda associamos um automorfismo do shift chamado automorfismo de poda. Este automorfismo, sob certas condições, estabelece um vínculo entre a dinâmica de um elemento da família de Hénon e a poda associada. Com estes conceitos, podemos provar a conjectura de poda para certos parâmetros reais na família de Hénon.

Teorema 1.3.4. Há dois conjuntos abertos disjuntos $A_{1}$ e $A_{2}$ no plano de parâmetros reais tal que se $(a, b) \in A_{1} \cup A_{2}$ então $H_{a, b}$ é topologicamente conjugado a uma poda não trivial da ferradura.

No capítulo 6 apresentamos alguns problemas de pesquisa e nova evidência que suporta a CFP. 


\section{Parte I}

Teoria de poda diferenciável 


\section{Capítulo 2}

\section{Conceitos}

Neste capítulo apresentamos os conceitos que utilizaremos nos capítulos subsequentes. Na seção 2.1 definimos algumas notações: o que é uma ferradura, o plano simbólico e hiperbolicidade. Na seção 2.2 mostramos propriedades de transformações de Hénon e na seção 2.3 lembramos as ideias fundamentais da teoria de poda desenvolvidas em [dC99].

\subsection{Preliminares}

\subsubsection{Terminologia e notações}

Denotaremos a homeomorfismos do plano pelas letras $f, g, F, G$ ou $H$.

Pontos do plano são denotados por $x, y, z$ e $p$.

Um disco topológico é um domínio aberto do plano simplesmente conexo.

Denotaremos por Id à identidade $\operatorname{Id}(x)=x, \forall x \in \mathbb{R}^{2}$.

Um ponto $x$ é chamado $n$-periódico sob um homeomorfismo $f$ se existe um $n \in \mathbb{N}$ tal que $f^{n}(x)=x$. O menor $n$ satisfazendo a anterior equação é chamado período. O conjunto de pontos periódicos será denotado por $\operatorname{Per}(f)$. A órbita de um ponto $x$ por $f$, denotada por $\operatorname{Orb}(x)=\operatorname{Orb}_{f}(x)$, é o conjunto $\left\{f^{n}(x)\right\}_{n \in \mathbb{Z}}$.

Dizemos que um conjunto $\Lambda$ é $f$-invariante se $\Lambda=f(\Lambda)=f^{-1}(\Lambda)$.

Um ponto $x \in \mathbb{R}^{2}$ é errante de $f$ se existe uma vizinhança $V$ tal que $f^{k}(V) \cap$ $V=\emptyset, \forall k$ positivo. Um ponto é chamado não errante se o anterior não é verdade, isto é, se para toda vizinhança $V$ de $x$, existe um inteiro positivo $k$ tal que $f^{k}(V) \cap V \neq \emptyset$. Denotamos o conjunto de pontos não errantes de $f$ por $\Omega(f)$.

Um ponto $x$ é recorrente por cadeias se, dado qualquer $\epsilon>0$, podemos escolher $N+1$ pontos $\left\{x_{i}\right\}_{i=1}^{N+1} \operatorname{com} x_{1}=x_{N+1}=x$ e $\left|x_{i+1}-f\left(x_{i}\right)\right|<\epsilon$, para $1 \leq i \leq N$. O conjunto de todos pontos recorrentes por cadeias é denotado por $\mathcal{R}(f)$.

Da definição temos que, para todo $f, \Omega(f)$ e $\mathcal{R}(f)$ são conjuntos fechados $f$-invariantes e vale que $\Omega(f) \subset \mathcal{R}(f)$. Além disso, $\Omega(f)=\Omega\left(f^{-1}\right)$ e $\mathcal{R}(f)=$ $\mathcal{R}\left(f^{-1}\right)$.

Duas transformações $F: X \rightarrow X$ e $G: Y \rightarrow Y$ de espaços topologicos são topologicamente conjugados se existe um homeomorfismo $h: X \rightarrow Y$ tal que

$$
h(F(x)))=G(h(x)), \text { para todo } x \in X .
$$


O homeomorfismo $h$ é chamado conjugação. Se $h$ é só sobrejetor dizemos que é uma semi-conjugação; neste caso dizemos que $F$ é um fator de $G$.

Denotaremos por Diff ${ }^{r}\left(\mathbb{R}^{2}\right)$ ao conjunto de todos os $C^{r}$-difeomorfismos de $\mathbb{R}^{2}$, para $r \geq 1$, e a $C^{r}$-norma por $d_{C^{r}}$ para $r \geq 1$. A $C^{0}$-norma é a norma do supremo. Um $C^{r}$-difeomorfismo $f$ é dito $C^{m}$-estruturalmente estável $(1 \leq$ $m \leq r)$ se existe uma vizinhança $\mathcal{U}$ de $f$ na topologia $C^{m}$ tal que todo $g \in \mathcal{U}$ é topologicamente conjugado a $f$.

\subsubsection{Subshifts de tipo finito}

Seja $[k]=\{0, \ldots, \mathrm{k}-1\}$ o espaço topológico de $k$ símbolos com a topologia discreta. Seja $\Sigma_{k}=[k]^{\mathbb{Z}}$ o espaço produto. Um elemento de $\Sigma_{k}$ é uma sequência bi-infinita de símbolos de $[k], a=\ldots a_{-1} \cdot a_{0} a_{1} \ldots$. Quando escrevemos elementos $a \in \Sigma_{k}$ é comum colocar um ponto '.' entre $a_{-1}$ e $a_{0}$ para indicar a origem da sequência. Note que a topologia produto é induzida pela métrica

$$
d(a, b)=\sum_{n=-\infty}^{\infty} 2^{-(2|n|+1)}\left|a_{n}-b_{n}\right|
$$

O conjunto $\Sigma_{k}$ é compacto, totalmente desconexo e não têm pontos isolados, logo é um conjunto de Cantor. O homeomorfismo $\sigma: \Sigma_{k} \rightarrow \Sigma_{k}$ tal que $(\sigma(a))_{i}=a_{i+1}$ será chamado o shift.

Seja $M_{k}$ o conjunto das matrizes $k \times k$ cujas entradas são 1 ou 0 e seja $A=\left(A_{i, j}\right) \in M_{k}$. Definimos $\Sigma_{A}=\left\{a \in \Sigma_{k}: \forall i, A_{a_{i} a_{i+1}}=1\right\} . \quad \Sigma_{A}$ é um subespaço fechado e $\sigma$-invariante de $\Sigma_{k}$. Denotamos $\sigma_{A}=\left.\sigma\right|_{\Sigma_{A}}$. O par $\left(\Sigma_{A}, \sigma_{A}\right)$ é chamado um subshift de tipo finito.

Se $a=a_{1} \ldots a_{n}$ é uma sequência finita admissível de símbolos tal que $a_{n} a_{1}$ é permitida pela matriz $A$, ou seja $A_{a_{n} a_{1}}=1$, então $a^{\infty}$ é a repetição bi-infinita da sequência $a$.

Utilizaremos $s=\left(s_{i}\right)_{i \in \mathbb{Z}}$ para denotar um elemento de $\Sigma_{2}$.

Definições anâlogas se podem fazer para $\Sigma_{k}^{+}=[k]^{\mathbb{N}}$. Neste caso os shifts ou subshifts são chamados unilaterais.

\subsubsection{A ferradura de Smale}

Um dos homeomorfismos do plano com dinâmica não trivial que pode ser compreendida completamente é a Ferradura de Smale F definida por Stephen Smale em [Sma67]. Este pode ser construido como segue. Considere o quadrado $Q=[-1,1] \times[-1,1]$ unido a dois semi-discos de raio 1 centrados em $(-1,0)$ e $(1,0)$. Veja a figura 2.1. Denotemos esta região por $\mathbb{T}$. A ação de $F$ em $\mathbb{T}$ é esticar $Q$ uniformemente na direção horizontal por um fator $\lambda$ (o qual deve ser maior que 2), contrair o resultado na direção vertical por um fator $1 / \lambda$ e então dobrar como na figura 2.1 de modo que segmentos verticais em $Q \cap F^{-1}(Q)$ são levados dentro de segmentos verticais por $F$ e segmentos horizontais em $Q \cap F^{-1}(Q)$ são levados dentro de segmentos horizontais por $F^{-1}$. Os dois semi-discos são contraidos uniformemente e levados no interior do semi-disco da esquerda. Fora de $\mathbb{T}, F$ tem um ponto fixo no $\infty \operatorname{com} F^{-n}(x) \rightarrow \infty$ quando $n \rightarrow \infty$ para todo $x \notin \mathbb{T}$. 


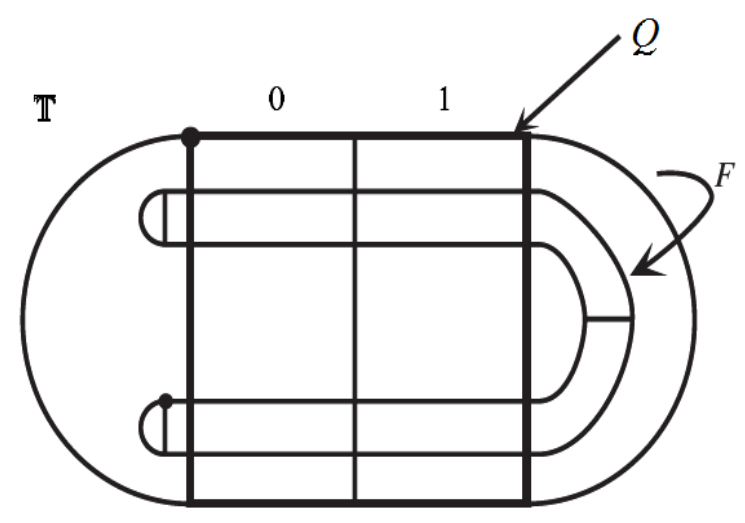

Figura 2.1: Dinâmica da Ferradura $F$ [dCH02b].

O conjunto não-errante $\Omega(F)$ de $F$ é a união de $\infty$, um ponto atrator no semi-disco da esquerda e um conjunto de Cantor

$$
\Lambda=\left\{x \in \mathbb{R}^{2}: F^{n}(x) \in Q, \text { para todo } n \in \mathbb{Z}\right\} .
$$

A dinâmica de $F$ em $\Lambda$ pode ser compreendida simbolicamente: seja $\pi_{1}: \mathbb{T} \rightarrow I$ a projeção na primeira coordenada e defina o itinerário $h: \Lambda \rightarrow \Sigma_{2}$ por

$$
(h(x))_{i}= \begin{cases}0 & \text { se } \pi_{1}\left(F^{i}(x)\right)<0, \\ 1 & \text { se } \pi_{1}\left(F^{i}(x)\right)>0 .\end{cases}
$$

Assim, $h$ é um homeomorfismo que conjuga a dinâmica de $F$, restrita a $\Lambda$, com a dinâmica do shift $\sigma: \Sigma_{2} \rightarrow \Sigma_{2}$. Em outras palavras

$$
h(F(x))=\sigma(h(x)), \text { para todo } x \in \Lambda .
$$

De fato, a ferradura que consideraremos é uma variação de $F$, chamada também $F$, obtida fazendo uma primeira homotopia que colapsa o bordo de $\mathbb{T}$ no $\infty$ e depois uma segunda homotopia que leva o ponto fixo no semi-disco da esquerda no $\infty$, deixando o conjunto $\Lambda$ intacto. Assim, $\Lambda$ pode ser caracterizado por

$$
\Lambda=\left\{x \in \mathbb{R}^{2}:\left\{F^{n}(x)\right\}_{n \in \mathbb{Z}} \text { é limitada }\right\}
$$

ou o conjunto de pontos cujas órbitas estão contidas em $Q$.

Sem perda de generalidade podemos supor que $F$ é um $C^{\infty}$-difeomorfismo do plano. 


\subsubsection{Plano simbólico}

Em [Cvi91, CGP88], Cvitanović, Gunaratne e Proccacia utilizaram o plano simbólico para representar uma órbita de uma transformação de Hénon. No que segue mostraremos a construção do plano simbólico a partir das órbitas de $\Sigma_{2}$ que, essencialmente, é a imagem de uma aplicação $\zeta: \Sigma_{2} \rightarrow[0,1]^{2}$ que colapsa as lacunas entre as variedades invariantes da ferradura.

Denote $\mathbb{Z}_{+}=\{n \in \mathbb{Z}: n \geq 0\}$ e $\mathbb{Z}_{-}=\{n \in \mathbb{Z}: n<0\}$ e sejam os conjuntos $\Sigma^{+}=\{0,1\}^{\mathbb{Z}_{+}}$e $\Sigma^{-}=\{0,1\}^{\mathbb{Z}_{-}}$. É claro que $\Sigma_{2}=\Sigma^{-} \times \Sigma^{+}$. Defina uma ordem $\prec$ no conjunto $\{0,1\}$ tal que $0 \prec 1$.

A ordem horizontal e vertical de pontos em $\Sigma_{2}$ pode ser determinado usando a ordem unimodal $\preceq$ em $\Sigma^{+}$, a qual é definida como segue (veja [Dev89,MT88]).

Definição 2.1.1. Sejam $s=s_{0} s_{1} \ldots$ e $t=t_{0} t_{1} \ldots$ elementos distintos de $\Sigma^{+}$e $n \in \mathbb{Z}_{+}$o menor natural tal que $s_{n} \neq t_{n}$, então $s \prec t$ se e somente se $\sum_{i=0}^{n} s_{i}$ é par. Isto é equivalente a dizer que

(i) $\sum_{i=0}^{n-1} s_{i}$ é par e $s_{n} \prec t_{n}$, ou

(ii) $\sum_{i=0}^{n-1} s_{i}$ é impar e $t_{n} \prec s_{n}$.

Se $s, t \in \Sigma^{+}$, então $s \preceq t$ se e somente se ou $s=t$ ou $s \prec t$.

Sabemos que $\Sigma^{+}$define um conjunto de Cantor no intervalo $[0,1]$ e que existe uma aplicação $\alpha: \Sigma^{+} \rightarrow[0,1]$ que colapsa as lacunas deste conjunto de Cantor. $\alpha$ é construida do seguinte modo (veja [CGP88, HC98]):

Para cada $s \in \Sigma^{+}$definimos os números

$$
c_{n}:=\sum_{i=0}^{n} s_{i} \bmod 2
$$

Ou equivalentemente, se $c_{0}=s_{0}$, então, para $n \geq 1$ :

$$
c_{n}:=\left\{\begin{array}{cc}
c_{n-1} & \text { se } s_{n}=0 \\
1-c_{n-1} & \text { se } s_{n}=1
\end{array}\right.
$$

Então, defina

$$
\alpha(s)=0 . c_{1} c_{2} \ldots=\sum_{n=0}^{\infty} \frac{c_{n}}{2^{n+1}}
$$

Observe que $\alpha$ preserva a ordem unimodal em $\Sigma^{+}$e colapsa as sequências $s \in \Sigma^{+}$ que são pre-imagens de $0^{\infty}$ pelo $\sigma$, no seguinte sentido:

Proposição 2.1.1. Sejam $s, t \in \Sigma^{+}$tais que $s \prec t$ então $\alpha(s) \leq \alpha(t)$. Se $\alpha(s)=$ $\alpha(t)$ então existe um $N \in \mathbb{N}$ tal que $s=s_{0} s_{1} \ldots s_{N} 010^{\infty}$ e $t=s_{0} s_{1} . . s_{N} 110^{\infty}$ se $\sum_{i=0}^{N} s_{i}$ é par, et $=s_{0} s_{1} \ldots s_{N} 010^{\infty}$ e $s=s_{0} s_{1} . . s_{N} 110^{\infty}$ se $\sum_{i=0}^{N} s_{i}$ é impar.

Observação 2.1.1. Note que se $\alpha(s)=\alpha(t)$ então $s$ e $t$ são consecutivos na ordem unimodal, isto é, não existe sequência $s^{\prime} \in \Sigma^{+}$tal que $s \prec s^{\prime} \prec t$ ou $t \prec s^{\prime} \prec t$. 
Para todo $s=\left(s_{i}\right)_{i=-\infty}^{\infty} \in \Sigma_{2}$ definimos as coordenadas no plano simbólico de $s, \zeta(s) \in[0,1] \times[0,1]$, por:

$$
\zeta(s)=\left(\alpha\left(s_{0} s_{1} \ldots\right), \alpha\left(s_{-1} s_{-2} \ldots\right)\right)
$$

Definição 2.1.2. Suponha que o conjunto não errante $\Omega(f)$ de $f$ é equivalente a um subconjunto de $\Sigma_{2}$, isto é, cada ponto $p \in \Omega(f)$ tem uma descrição simbólica $s(p) \in \Sigma_{2}$. Então o Plano Simbólico $S(f)$ de $f$ é o subconjunto de $[0,1] \times[0,1]$ definido por

$$
S(f)=\{\zeta(s(p)): p \in \Omega(f)\}
$$

\subsubsection{Hiperbolicidade}

Definição 2.1.3. Seja $\Lambda$ um conjunto invariante para um $C^{r}$-difeomorfismo $f$ de uma variedade $M$. Dizemos que $\Lambda$ é um conjunto hiperbólico se existe uma decomposição contínua do espaço tangente de $M$ restrito a $\Lambda, T M_{\Lambda}$, a qual é invariante por $T f$

$$
T M_{\Lambda}=E^{s} \oplus E^{u} ; T f\left(E^{s}\right)=E^{s} ; T f\left(E^{u}\right)=E^{u} ;
$$

e para a qual existem constantes $C$ e $\lambda, C>0$ e $0<\lambda<1$, tais que para todo $n \geq 0$,

$$
\begin{gathered}
\|\left. T f^{n}\right|_{E^{s}}||<C \lambda^{n}, \\
\left\|\left.T f^{-n}\right|_{E^{u}}\right\|<C \lambda^{n} .
\end{gathered}
$$

Definição 2.1.4. Dado um ponto $x$ da variedade $M$, os conjuntos estável e instável locais de um difeomorfismo $f$ em $x$ são:

$$
\begin{gathered}
W_{\epsilon}^{s}(x, f):=\left\{y \in M \mid \lim _{n \rightarrow \infty} d\left(f^{n}(x), f^{n}(y)\right)=0 \text { e } d\left(f^{n}(x), f^{n}(y)\right) \leq \epsilon, \forall n \geq 0\right\} \\
W_{\epsilon}^{u}(x, f):=\left\{y \in M \mid \lim _{n \rightarrow-\infty} d\left(f^{n}(x), f^{n}(y)\right)=0 \text { e } d\left(f^{n}(x), f^{n}(y)\right) \leq \epsilon, \forall n \leq 0\right\}
\end{gathered}
$$

Assim as variedades estáveis e instáveis são

$$
\begin{aligned}
W^{s}(x, f) & :=\cup_{n \geq 0} f^{-n} W_{\epsilon}^{s}\left(f^{n}(x)\right) \\
W^{u}(x, f) & :=\cup_{n \geq 0} f^{n} W_{\epsilon}^{u}\left(f^{-n}(x)\right)
\end{aligned}
$$

Sabese que quando $\Lambda$ é hiperbólico, para cada $x \in \Lambda, W_{\epsilon}^{s}(x, f)$ e $W_{\epsilon}^{u}(x, f)$ são variedades mergulhadas em $M$ que passam por $x$. Ver, por exemplo, [Shu87, Teorema 6.2]:

Teorema 2.1.1. Seja $\Lambda$ um conjunto hiperbólico fechado para $f$. Então existe um número positivo $\epsilon$ tal que para cada ponto $x \in \Lambda, W_{\epsilon}^{s}(x, f)$ é um disco topológico de igual dimensão à de $E^{s}$; além disso, $T_{x} W^{s}(x, f(x))=E_{x}^{s}$; uma similar definição se cumpre para $W_{\epsilon}^{u}(x, f)$. Se $f$ é $C^{r}$ então $W_{\epsilon}^{s}(x, f)$ e $W_{\epsilon}^{u}(x, f)$ são $C^{r}$-variedades.

A hiperbolicidade permite obter resultados sobre estabilidade estrutural. Em 
[KH95, Teorema 18.2.1] demonstra-se que a hiperbolicidade em um conjunto invariante $\Lambda$ é suficiente para garantir que $\left.f\right|_{\Lambda}$ é estruturalmente estável.

Teorema 2.1.2. Seja $\Lambda \subset M$ um conjunto hiperbólico de um difeomorfismo $f: U \rightarrow M$. Então para toda vizinhança aberta $V \subset U$ de $\Lambda$ e cada $\delta>0$, existe $\epsilon>0$ tal que se $g: U \rightarrow M$ e $d_{C^{1}}\left(\left.f\right|_{V}, g\right)<\epsilon$, existe um conjunto hiperbólico $\Lambda^{\prime}=g\left(\Lambda^{\prime}\right) \subset V$ para $g$ e um homeomorfismo $h: \Lambda^{\prime} \rightarrow \Lambda$ com $d_{C^{0}}(I d, h)+$ $d_{C^{0}}\left(I d, h^{-1}\right)<\delta$ tal que

$$
\left.h \circ g\right|_{\Lambda^{\prime}}=\left.f\right|_{\Lambda} \circ g .
$$

Além disso h é única quando $\delta$ é pequeno.

\subsubsection{Partições de Markov}

Uma partição de Markov é um conjunto de retângulos no plano que permite introduzir dinâmica simbólica num conjunto invariante $\Lambda$ para um difeomorfismo do plano.

Definição 2.1.5. Uma partição de Markov [PT93] para um conjunto $\Lambda$ de un difeomorfismo $f$ consiste de um conjunto $\mathcal{P}$ de retângulos, isto é, imagens difeomorfas ao quadrado $Q=[-1,+1]^{2}$, denotadas por $B_{1}=\Psi_{1}(Q), B_{2}=$ $\Psi_{2}(Q), \ldots, B_{l}=\Psi_{l}(Q)$ tais que:

(i) $\Lambda \subset \bigcup_{i=1}^{l} B_{i}$

(ii) $\operatorname{int}\left(B_{i}\right) \cap \operatorname{int}\left(B_{j}\right)=\emptyset$, se $i \neq j$, onde $\operatorname{int}(B)$ denota o interior de $B$.

$$
f\left(\partial_{s} B_{i}\right) \subset \bigcup_{j=1}^{l} \partial_{s} B_{j}
$$

e

$$
f^{-1}\left(\partial_{u} B_{i}\right) \subset \bigcup_{j=1}^{l} \partial_{u} B_{j}
$$

onde $\partial_{s} B_{i}=\Psi(\{(x, y)|| x \mid=1,-1 \leq y \leq 1\})$ é o lado estável do retângulo $B_{i}$ e $\partial_{u} B_{i}=\Psi(\{(x, y) \| y \mid=1,-1 \leq x \leq 1\})$ é o lado instável de $B_{i}$.

O [KH95, teorema 18.7.3] diz que todo conjunto hiperbólico possui uma partição de Markov:

Teorema 2.1.3. Um conjunto compacto invariante e hiperbólico $\Lambda$ admite uma partição de Markov de diametro arbitrariamente pequeno.

A principal consequência do teorema anterior é a existência de uma semiconjugação entre o conjunto hiperbólico e um subshift de tipo finito. Isto é o teorema [KH95, Teorema 18.7.4]:

Teorema 2.1.4. Suponha que $\Lambda$ é um conjunto maximal invariante hiperbólico $e \mathcal{R}=\left\{R_{1}, \ldots, R_{l}\right\}$ uma partição de Markov de diametro suficientemente pequeno $e$

$$
A_{i, j}:=\left\{\begin{array}{cc}
1 & \text { se } R_{i} \cap f^{-1}\left(R_{j}\right) \neq \emptyset \\
0 & \text { em outro caso }
\end{array}\right.
$$


Então $\left.f\right|_{\Lambda}$ é um fator do subshift de tipo finito $\left(\Sigma_{A}, \sigma_{A}\right)$.

Uma pregunta que aparece, então, é quando a semiconjugação do teorema anterior é uma conjugação. Note que para isso é necessario que $\Lambda$ seja totalmente disconexo, dado que $\Sigma_{A}$ é totalmente desconexo. O seguinte teorema ( [KH95, Teorema 18.7.8]) diz que, na verdade, isto é uma condição suficiente.

Teorema 2.1.5. Seja $\Lambda$ um conjunto totalmente desconexo compacto maximal e hiperbólico para um difeomorfismo $f$. Então $\left.f\right|_{\Lambda}$ é topologicamente conjugado ao subsfhit $\left(\Sigma_{A}, \sigma_{A}\right)$.

\subsection{Propriedades de transformações de Hénon}

A família de transformações de Hénon [Hén76] tem sido muito estudada em sistemas dinâmicos. Ela é dada por

$$
H_{a, b}(x, y)=\left(a-x^{2}-b y, x\right)
$$

onde $a, b \in \mathbb{R}$. Fixados $(a, b)$ no contexto, simplesmentes dizemos $H$ em vez de $H_{a, b}$. Note que $\operatorname{Jac}(H)=b$. Estaremos interessados principalmente no caso $b>0$, no qual $H_{a, b}$ preserva orientação.

Para um número positivo $R$, definimos os conjuntos:

$$
\begin{aligned}
V_{R}:= & \left\{(x, y) \in \mathbb{R}^{2} \mid \max \{|x|,|y|\}<R\right\}, \\
V_{R}^{+}:= & \left\{(x, y) \in \mathbb{R}^{2}|\max \{R,|y|\}<| x \mid\right\}, \\
V_{R}^{-}:= & \left\{(x, y) \in \mathbb{R}^{2}|\max \{R,|x|\}<| y \mid\right\}, \\
& \Lambda_{R}(H):=\bigcap_{n \in \mathbb{Z}} H^{n}\left(V_{R}\right),
\end{aligned}
$$

e o conjunto de órbitas limitadas

$$
K_{a, b}:=\left\{(x, y) \in \mathbb{R}^{2} \mid\left\{H_{a, b}^{n}(x, y)\right\}_{n \in \mathbb{Z}} \text { é limitada }\right\} .
$$

No que segue, não faremos referência ao número $R$ e simplesmente escreve$\operatorname{mos} V, V^{+}$and $V^{-}$.

Em [MNTU00], por exemplo, pode ser encontrada a seguinte proposição cuja demonstração é um simples cálculo utilizando a fórmula da transformação:

Proposição 2.2.1. Para $R \gg 0$, existe um número real $\rho>1$ com as seguintes propriedades:

(a) $S e(x, y) \in \overline{V^{+}}$e $H(x, y)=\left(x_{1}, y_{1}\right)$ então $H(x, y) \in V^{+} e\left|x_{1}\right|>\rho|x|$.

(b) $S e(x, y) \in \overline{V^{-}} e H^{-1}(x, y)=\left(x_{-1}, y_{-1}\right)$ então $H^{-1}(x, y) \in V^{-} e\left|y_{-1}\right|>$ $\rho|y|$.

Observação 2.2.1. Vamos estimar o número $R$ para a transformação de Hénon $H_{a, b}$. É suficiente escolher $R$ tal que $\left|a-\zeta^{2}\right|>(1+|b|)|\zeta|$, para $|\zeta| \geq R(\operatorname{com} \rho$ 
suficientemente próximo de 1). Dado que

$$
\left.\left|a-\zeta^{2}\right|-(1+|b|)|\zeta|\right) \geq|\zeta|^{2}-(1+|b|)|\zeta|-|a|
$$

concluimos que $R$ têm a propriedade procurada se é maior que a raiz positiva da equação: $t^{2}-(1+|b|) t-|a|=0$ (Compare com [DN79]). Uma descrição pictórica da anterior proposição é dada na figura 2.2.

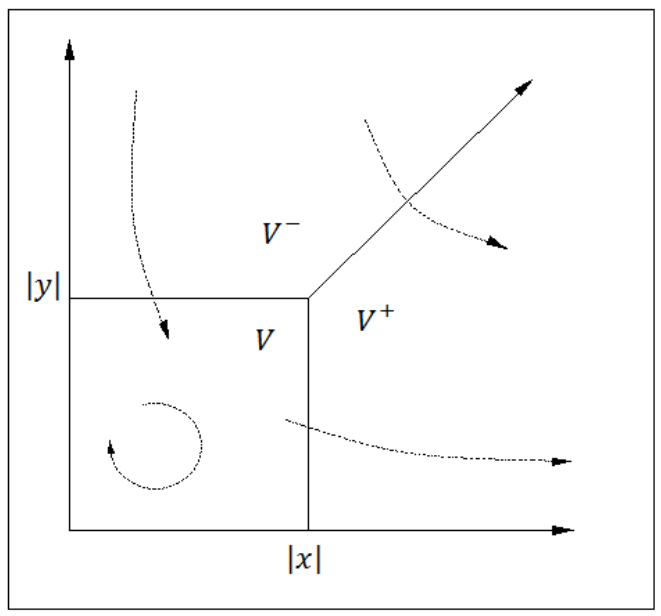

Figura 2.2: Dinâmica de uma transformação de Hénon.

Apresentamos outra importante proposição de [MNTU00] da qual daremos a prova pois será utilizada posteriormente.

Proposição 2.2.2. Seja $H$ uma transformação de Hénon, então para $R \gg 0$ temos que $\Omega(H) \subset V_{R}$.

Demonstração. Suponha que $z=(x, y) \in V^{+}$e seja $\left(x_{n}, y_{n}\right):=H^{n}(x, y), n \geq 0$. Por (a) da proposição 2.2.1 concluimos que:

$$
\left|x_{n}\right|>\rho\left|x_{n-1}\right|>\rho^{2}\left|x_{n-2}\right|>\ldots>\rho^{2}|x| \geq \rho^{n} R .
$$

Isso implica que, para $\epsilon>0$ suficientemente pequeno, é impossível tomar uma sequência $\left\{z_{n}\right\}_{n=1}^{N+1}$ com $z=z_{1}=z_{N+1}$ e $\left|z_{n+1}-H\left(z_{n}\right)\right|<\epsilon,(1 \leq n \leq N)$, o qual implica que $z \notin \mathcal{R}(H)$. Similarmente temos que se $z \in V^{-}$então $z \notin \mathcal{R}(H)$. Logo $\mathcal{R}(H) \subset V$.

Outra proposição importante é a seguinte:

Proposição 2.2.3. Se $H_{a, b}$ é uma transformação de Hénon e $R$ é suficientemente grande então $\Omega\left(H_{a, b}\right) \subset \Lambda_{R}\left(H_{a, b}\right)$ e $\Lambda_{R}\left(H_{a, b}\right)=K_{a, b}$.

Veja a [MNTU00, Proposição 9.2.6] para a primeira parte da proposição e [BS91, Lemma 2.4] para a segunda parte. 


\subsubsection{A ferraduras na família de Hénon}

Em [DN79], Devaney e Nitecki estudaram a família de Hénon e descobriram valores dos parâmetros nos quais a transformação de Hénon é uma ferradura de Smale.

Teorema 2.2.1 (Devaney-Nitecki). Se $a>\frac{5+2 \sqrt{5}}{4}(1+|b|)^{2}$ ent $\tilde{a} o \Omega\left(H_{a, b}\right)=$ $K_{a, b}, \Omega\left(H_{a, b}\right)$ tem uma estrutura hiperbólica e $\left.H_{a, b}\right|_{\Omega\left(H_{a, b}\right)}$ é topologicamente conjugada a $\left.\sigma\right|_{\Sigma_{2}}$.

Um resultado mais abrangente ao anterior pode-se encontrar na tese de Oberste-Vorth [OV87]. Veja também [MNTU00, Proposição 7.4.6].

Teorema 2.2.2 (Oberste-Vorth). Se $a>2(1+|b|)^{2}$ então $\Omega\left(H_{a, b}\right)=K_{a, b}$, $\Omega\left(H_{a, b}\right)$ tem uma estrutura hiperbólica e $\left.H_{a, b}\right|_{\Omega\left(H_{a, b}\right)}$ é topologicamente conjugada $\left.a \sigma\right|_{\Sigma_{2}}$.

Por estes dois teoremas, podemos escolher parâmetros $(a, b)$ tais que para $H:=H_{a, b}, \Omega(H)$ é hiperbólico e topologicamente conjugado à ferradura. Por tanto existe um homeomorfismo $h: \Omega(H) \rightarrow \Sigma_{2}$ tal que o seguinte diagrama comuta:

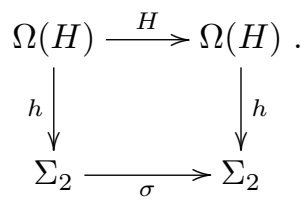

Assim cada ponto de $\Omega(H)$ corresponde a uma sequência bi-infinita de $\Sigma_{2}$. Uma órbita periódica de $H$ corresponde a uma sequência simbólica periódica $\left(s_{0} s_{1} \ldots s_{n-1}\right)^{\infty}$ onde $w^{\infty}$ significa a repetição bi-infinita da palavra w. Existem unicamente dois pontos fixos $0^{\infty}$ e $1^{\infty}$. Além disso, uma órbita homoclínica ao ponto $0^{\infty}$ é representada por uma sequência simbólica do tipo $0^{\infty} 1 s_{-T} \ldots s_{-1}$. $s_{0} s_{1} \ldots s_{N} 10^{\infty}$. Para cada sequência $s=\ldots s_{-1} \cdot s_{0} s_{1} \ldots$ definimos $x:=s_{0} s_{1} \ldots$ e $y:=\ldots s_{-2} s_{-1}$ e escrevemos $s=y \cdot x$. Doravante, um ponto em $z \in \Omega(H)$ será chamado por sua representação simbólica $h(z)$ e $\Lambda:=\Omega(H)$.

A conjugação $h: \Omega(H) \rightarrow \Sigma_{2}$ é escolhida como sendo a conjugação canônica. Isto é, para cada $z=(x, y)$ em $\Omega(H)$ associamos uma sequência simbólica $h(z)=\left(s_{i}\right)_{i \in \mathbb{Z}}$ definida por:

$$
s_{i}:= \begin{cases}0 & \text { se } \pi_{1}\left(H^{i}(z)\right)<0 \\ 1 & \text { se } \pi_{1}\left(H^{i}(z)\right)>0\end{cases}
$$

onde $\pi_{1}$ é a projeção na primeira coordenada $\pi_{1}(z):=x$.

Note que esta codificação é válida para transformações de Hénon tais que $a \gg 0$; por exemplo, por [DN79] temos

Proposição 2.2.4. $H_{a, b}$ é uma ferradura topológica se $a>2(1+|b|)^{2}$ e existe um $\delta>0$ tal que $\left|\pi_{1}(z)\right|>\delta$ para todo $z \in \Omega\left(H_{a, b}\right)$. 
Para outros parâmetros usaremos continuação hiperbólica quando seja necessário. Uma representação das variedades invariantes da ferradura é como na figura 2.3. O conjunto não errante é o fecho das interseções destas variedades.

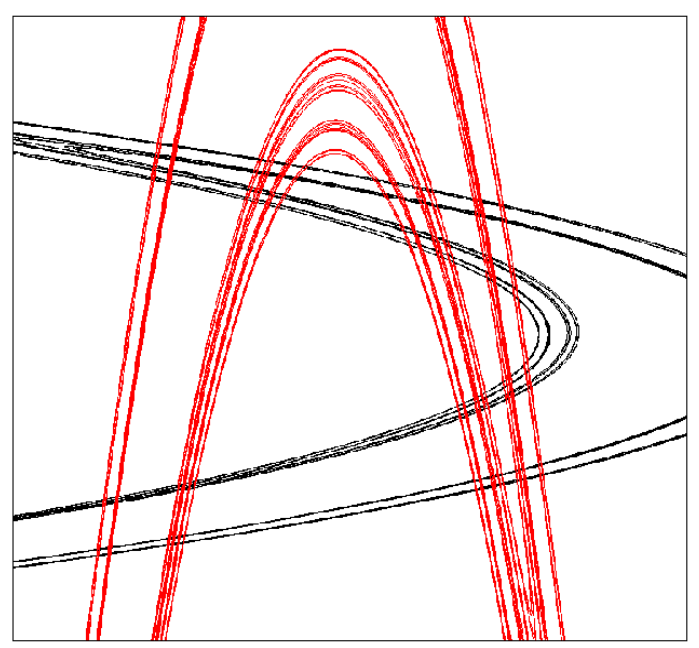

Figura 2.3: Variedades invariantes de uma transformação de Hénon que é uma ferradura de Smale $(a \approx 4.75$ e $b=0.75)$.

Outros importantes estudos sobre os parâmetros $(a, b)$ nos quais $H_{a, b}$ é uma ferradura hiperbólica foram feitos por Bedford e Smillie em [BS04,BS06b] e junto a Lyubich em [BLS93]. No que segue resumimos os seus principais resultados.

Primeiro observe que existe evidência numérica mostrando que a região $a>$ $2(1+|b|)^{2}$ do teorema de Oberste-Vorth não contém todas as ferraduras reais (veja [Ara07a]). Mas, para uma transformação de Hénon que seja uma ferradura hiperbólica, o fato que o conjunto de órbitas limitadas seja igual ao conjunto não errante é ainda válido. O seguinte teorema é uma das conclusões do [BLS93, Teorema 10.2]:

Teorema 2.2.3 (Bedford-Lyubich-Smillie). Se $\left.H_{a, b}\right|_{\Lambda}$ é uma ferradura completa real então $\Omega\left(H_{a, b}\right)=K_{a, b}$.

Dizemos que uma transformação de Hénon tem entropia maximal se a sua entropia topológica é igual a $\log (2)$. Em [BS04], Bedford e Smillie clasificaram as transformações de Hénon de entropia maximal. O seu principal resultado é:

Teorema 2.2.4 (Bedford-Smillie). Se $H_{a, b}$ tem entropia maximal então ou $H_{a, b}$ é hiperbólico e topologicamente conjugado a uma ferradura de Smale ou $H_{a, b}$ possui uma tangência quadrática.

Em [Hoe08], Ulrich Hoensch concluiu que se $H$ tem entropia maximal mas não é hiperbólica, ou seja o segundo caso no teorema anterior, a órbita de tangência homoclínica deve ocorrer entre as variedades estáveis e instáveis de 
um ponto fixo da ferradura. Se utilizarmos a codificação (2.26), o resultado de Hoensch se pode escrever como a seguinte proposição:

Proposição 2.2.5 (Hoensch). Se $H_{a, b}$ corresponde a uma transformação de Hénon com entropia maximal não hiperbólica então a tangência homoclínica ocorre quando as órbitas homoclínicas codificadas por $0^{\infty} 10 \cdot 10^{\infty}$ e $0^{\infty} 11 \cdot 10^{\infty}$ colidem.

Assim, a perda de hiperbolicidade de uma transformação de Hénon de entropia maximal é devida ao fato que as órbitas $0^{\infty} 10 \cdot 10^{\infty}$ e $0^{\infty} 11 \cdot 10^{\infty}$ colidem criando uma tangência homoclínica ao ponto fixo de codificação $0^{\infty}$. Um exemplo é como na figura 2.4. Ainda neste caso, o conjunto não errante é um conjunto de Cantor. Isto é o teorema 5.2 de [BS04]):

Teorema 2.2.5 (Bedford-Smillie). Se $H_{a, b}$ tem entropia maximal então $K_{a, b}$ é um conjunto de Cantor.

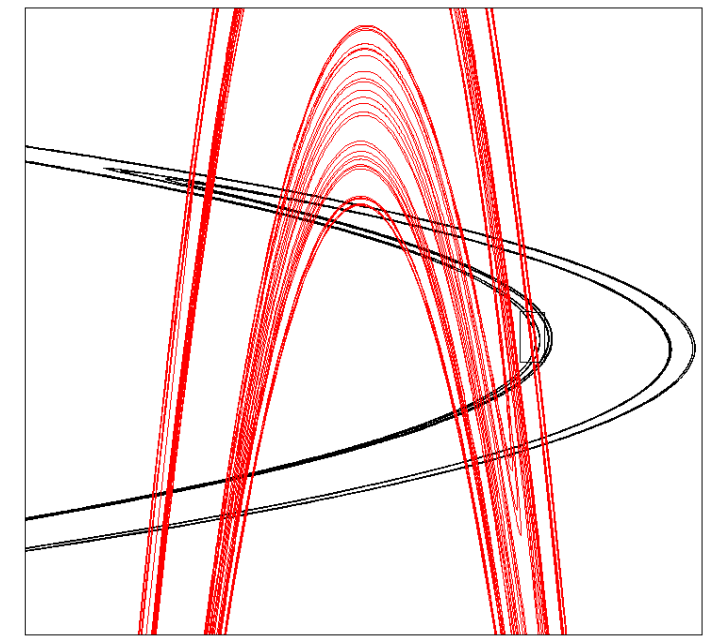

Figura 2.4: Variedades invariantes de uma transformação de Hénon com entropia maximal $(a \approx 3.3715$ e $b=0.5)$.

\subsection{Teoria da Frente de Poda}

\subsubsection{Evidência para a CFP}

Os primeiros estudos numéricos relacionados ao que, posteriormente, foi chamada Conjectura da Frente de Poda foram feitos por Cvitanović, Gunaratne e Procaccia em [CGP88]. Neste artigo, eles consideraram alguns valores dos parametros $^{1}(a, b)$ com $b<0$ e encontraram, numericamente, as órbitas periódicas de $H_{a, b}$ de períodos pequenos (menores que 15). Depois, desenharam elas no

\footnotetext{
${ }^{1}$ Em [CGP88] é utilizado uma família do tipo $C_{\mu, \epsilon}(x, y):=\left(y+\mu-x^{2}, \epsilon x\right) \operatorname{com} \epsilon>0$.
} 


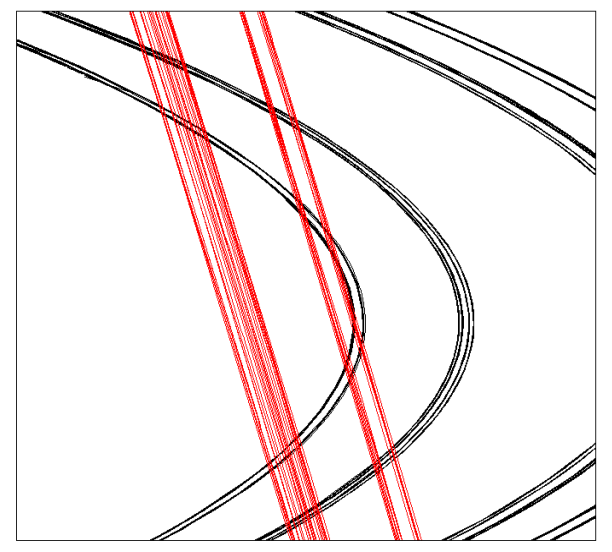

Figura 2.5: Zoom do retângulo na figura 2.4 que indica a regiâo onde está a tangência $(a \approx 3.3715$ e $b=0.5)$.

plano simbólico. Seus resultados sugeriram que o conjunto de órbitas periódicas não preenchia completamente o plano simbólico pois existiam regiões podadas que não correspondiam a dinâmica da transformação. Assim, eles conjecturaram que

o conjunto não-errante destas transformações corresponde a um subconjunto próprio do plano simbólico e, portanto, de $\Sigma_{2}$.

Usando o algoritmo do Apêndice A, nós reproduzimos alguns desses resultados. Embora nossa codificação simbólica difira um pouco da codificação em [CGP88] porque adaptamos a nossa apresentação do plano simbólico ao caso que $H_{a, b}$ preserva orientação, o espírito é o mesmo. Por exemplo, para os valores $(a, b)=$ $(1.9,0.05)$ o plano simbólico de $H_{a, b}$ para as órbitas de período menor que 15 é como na figura 2.6(b). Note que existem regiões no plano simbólico que não têm órbitas na dinâmica da transformação, ou seja, regiões podadas. Também desenhamos as variedades invariantes associadas a um ponto fixo (veja a figura 2.6(a)). É claro que a representação no plano simbólico é mais elucidativa, com respeito ao tipo de dinâmica que a transformação provavelmente tem, que a representação das variedades invariantes.

Em [Cvi91], Cvitanović introduziu o conceito de frentes de poda e formulou a Conjectura da Frente de Poda. Ele observou também que as regiões podadas se podem interpretar como o descruzamento de certas variedades invariantes estáveis e instáveis; assim, as órbitas podadas corresponderiam àquelas órbitas que estavam nas interseções entre estas variedades.

\subsubsection{Teoria de poda}

Uma formulação matematicamente rigorosa da Teoria de Poda foi desenvolvida por A. de Carvalho em [dC99]. Esta teoria permite destruir, de maneira controlada, parte da dinâmica de um homeomorfismo do superfície usando discos de poda. Neste contexto destruir dinâmica quer dizer converter os pontos 


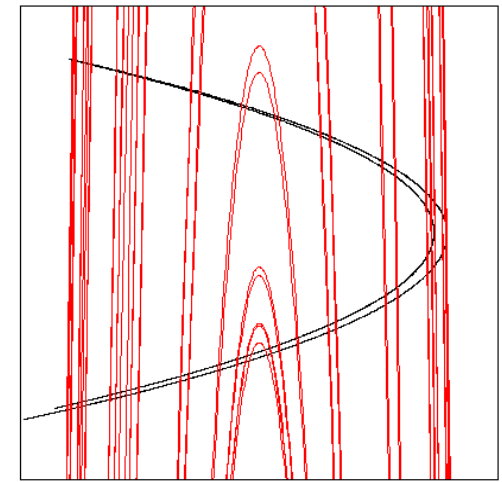

(a) Variedades estáveis e instáveis.

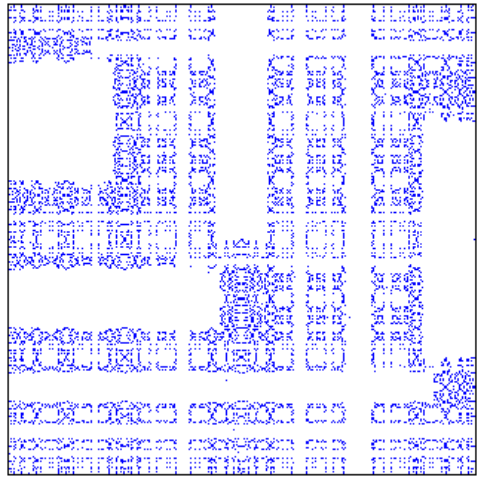

(b) Plano simbólico

Figura 2.6: Variedades estáveis e instáveis (Figura 2.6(a)) de $H_{1.9,0.05}$ e o plano simbólico para órbitas de períodos menores que 15 (Figura 2.6(b)).

não errantes de certas regiões em pontos errantes, sem modificar a dinâmica em outras regiões. Esta destruição é feita por uma isotopia suportada nos discos de poda.

Definição 2.3.1. Uma isotopia é uma transformação continua $H: \mathbb{R}^{2} \times[0,1] \rightarrow$ $\mathbb{R}^{2}$ tal que para todo $t \in[0,1], H_{t}:=H(\cdot, t)$ é um homeomorfismo do plano. Dizemos que $H$ é uma isotopia de $f$ se $H_{0}=f$. O suporte de $H$ é o complementar do conjunto $\{x: H(x, t)=H(x, 0), \forall t \in[0,1]\}$.

Seguindo as definições de [dC99], dado um homeomorfismo do plano $f$, um disco topologico $D$ é um $(c, e)$-disco para $f$ ( $c$ para "contractor"e $e$ para "expansor") se seu bordo pode ser representado como uma união de dois arcos que só se intersectam nos extremos, $\partial D=C \cup E$, satisfazendo as seguintes condições:

$$
\begin{aligned}
& \lim _{n \rightarrow \infty} \operatorname{diam} f^{n}(C)=0 \\
& \lim _{n \rightarrow \infty} \operatorname{diam} f^{-n}(E)=0
\end{aligned}
$$

Mais precisamente, sob certas condicões de diferenciabilidade para $f$, se pode pensar um $(c, e)$-disco de poda como sendo um aberto do plano limitado por dois $\operatorname{arcos} C$ e $E$ tais que $C$ pertence à variedade estável de um ponto hiperbólico e $E$ pertence à variedade instável de outro ponto hiperbólico.

A partir dos $(c, e)$-discos definimos o conceito de frentes de poda. Primeiro precisamos uma definição de ordem parcial sobre eles.

Definição 2.3.2. Sejam $D_{1}, D_{2}$ dois $(c, e)$-discos tais que $I_{1} \cap I_{2} \neq \emptyset$ onde $I_{i}=\operatorname{int}\left(D_{i}\right)$. Dizemos que $D_{1}$ é mais longo que $D_{2}$, o que denotamos por $D_{1} \succ D_{2}$, se as seguintes condições se cumprem (veja a figura 2.7):

i) $C_{1} \cap I_{2}=\emptyset$ e $E_{2} \cap I_{1}=\emptyset$; 


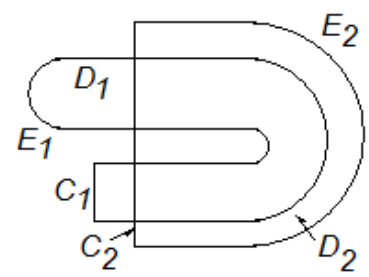

i)

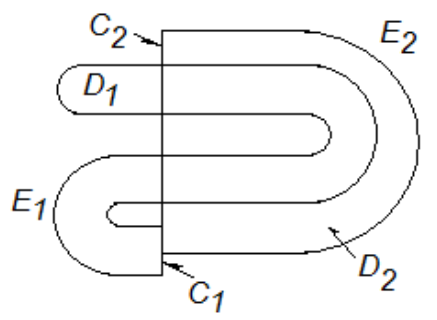

ii)

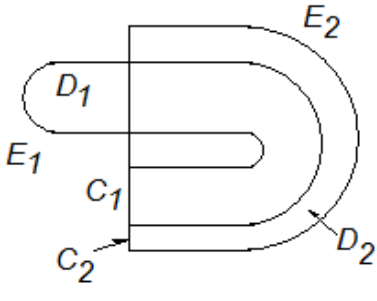

iii)

Figura 2.7: Relação $D_{1} \succ D_{2}$.

ii) se $C_{1} \cap C_{2} \neq \emptyset$ então $C_{1} \cup C_{2}$ é um arco e se $E_{1} \cap E_{2} \neq \emptyset$ então $E_{1} \cup E_{2}$ é um arco;

iii) se $C_{1}^{\circ} \cap \overline{I_{1} \cap I_{2}} \neq \emptyset$ então $C_{1} \subset C_{2}$ e se $E_{2}^{\circ} \cap \overline{I_{1} \cap I_{2}} \neq \emptyset$ então $E_{2} \subset E_{1}$.

O objetivo principal é mostrar como destruir dinâmica de uma maneira controlada. Em [dC99] são definidos conjuntos para os quais é possível fazer isso. Estes conjuntos foram chamados frentes de poda.

Definição 2.3.3. Seja $\left\{D_{i}\right\}_{i=1}^{l}$ uma coleção de $(c, e)$-discos tais que as seguintes condições são satisfeitas:

i) $\succ$ pode ser extendida por transitividade a um ordem parcial em $\left\{D_{i}\right\}_{i=1}^{l}$ ou, equivalentemente, não há laços $D_{i_{1}} \succ D_{i_{2}} \succ \ldots \succ D_{i_{n}} \succ D_{i_{1}}$;

ii) para cada $n>0$ e $i, j \in \underline{l}$, ou $f^{n}\left(D_{i}\right) \cap D_{j}=\emptyset$ ou $f^{n}\left(D_{i}\right) \succ D_{j}$;

iii) para cada $m<0$ e $i, j \in \underline{l}$, ou $f^{m}\left(D_{i}\right) \cap D_{j}=\emptyset$ ou $D_{j} \succ f^{m}\left(D_{j}\right)$.

Toda coleção satisfazendo as propriedades anteriores será chamada coleção de poda e a sua união $\cup_{i=1}^{l} D_{i}$ a frente de poda. O principal teorema de [dC99] é o teorema de poda:

Teorema 2.3.1 (de Carvalho). Seja $f: \mathbb{R}^{2} \rightarrow \mathbb{R}^{2}$ um homeomorfismo do plano, $\mathcal{D}=\left\{D_{i}\right\}_{i=1}^{l}$ uma coleção de discos de poda e $P=\bigcup_{i=1}^{l} I_{i}$, onde $I_{i}$ é o interior do disco $D_{i}$. Então existe uma isotopia $S: \mathbb{R}^{2} \times[0,1] \rightarrow \mathbb{R}^{2}$ da identidade, suportada em $U:=\bigcup_{k \in \mathbb{Z}} f^{k}(P)$ tal que se $f_{P}()=.f \circ S(., 1)$ então cada ponto de $U$ é errante sob $f_{P}$.

A transformação $f_{P}$ ao final da isotopia do teorema 2.3.1 é chamada uma poda de $f$. Dado que a isotopia é suportada em $U$ e que cada ponto de $U$ é errante para $f_{P}$, temos que a dinâmica interessante de $f_{P}$ está em $U^{c}$ e coincide ali com a dinâmica de $f$. Então, o teorema 2.3.1 permite destruir toda a dinâmica de $f$ no interior de $U$ por uma isotopia que mantém intacta $f$ no complemento de $U$.

O teorema de poda permite também construir a família de poda $\mathcal{P}(f)$ de um homeomorfismo $f$ da seguinte maneira. Suponha que $f$ possui um disco de poda 
$D_{1}$ então construa a poda $f_{D_{1}}$. Se $f_{D_{1}}$ novamente têm um disco de poda $D_{2}$ então podemos contruir uma poda de $f_{D_{1}}$ denotada por $f_{D_{1} D_{2}}$. Se for o caso, podemos continuar este processo até obter uma transformação $f_{D_{1} D_{2} \ldots D_{n}}$ para um $n$ apropiado. A família de poda de $f$ é o conjunto de todas as transformações obtidas desta maneira

$$
\mathcal{P}(f)=\overline{\left\{f_{D_{1} . . D_{n}}: D_{i+1} \text { é um disco de poda para } f_{D_{i}}\right\}}
$$

onde o fecho é tomado na topologia $C^{0}$. Nesta linguagem a Conjectura da Frente de Poda de Cvitanović é:

Conjectura da Frente de Poda: Seja $F$ a ferradura de Smale. Dado $(a, b) \in \mathbb{R}^{2}$, existe uma transformação de poda $g \in \mathcal{P}(F)$ que é semiconjugada a $H_{a, b}$.

Num trabalho não publicado J. Dillon [Dil] encontrou numéricamente valores dos parâmetros nos quais a anterior conjectura parece ser verdade.

Uma das dificultades que aparece ao tentar utilizar o teorema de poda anterior para mostrar a CFP é que, dado um disco de poda, a transformação de poda $f_{P}$ é obtida fazendo infinitas isotopias com suportes disjuntos. Isto faz que seja dificil trabalhar com a expressão da transformação de poda. Assim, é difícil saber qual é a dinâmica restante no conjunto não errante de $F_{P}$ no caso geral. Outra dificultade é que a transformação de poda é construída como sendo um homeomorfismo enquanto que a família de Hénon é uma família analítica de transformações. Isto faz que as possíveis comparações entre elas sejam dificiles de fazer.

No seguinte capítulo apresentaremos uma teoria de poda diferenciável utilizando discos de poda homoclínicos. 


\section{Capítulo 3}

\section{Teoria de poda na ferradura}

Em [dC99] poda foi introduzida como uma ferramenta topológica mas as transformações de poda dadas pelo teorema 2.3 .1 em geral não são diferenciáveis.

Neste capítulo demonstramos um teorema de poda diferenciável para o caso particular da ferradura de Smale construindo podas que são difeomorfismos. Para tal propósito, definimos os discos de poda homoclínicos os quais permitem construir uma deformação da ferradura que destrói a dinâmica suportada neles. Provaremos que esta deformação, obtida por uma difeotopia e chamada difeomorfismo de poda, é hiperbólica e possui uma dinâmica no conjunto não errante equivalente a um subshift de tipo finito.

Na seção 3.1 definimos o que é um disco de poda homoclínico e construimos as isotopias para o difeomorfismo de poda. Na seção 3.2 apresentamos um algoritmo que, utilizando as coordenadas do disco de poda, permite encontrar a partição de Markov do difeomorfismo de poda. Já na seção 3.3 demonstramos o teorema de poda.

\subsection{Construção do difeomorfismo de poda}

Pelo teorema 2.2.2 escolhemos parâmetros $(a, b)$ tais que $H_{a, b}$ é uma ferradura de Smale hiperbólica. Doravante $H_{a, b}$ será chamada simplesmente por $H$ e um ponto em $\Omega(H)$ será representado pela sua codificação simbólica em $\Sigma_{2}$.

\subsubsection{Frentes de poda e discos de poda homoclínicos}

Agora definiremos o conceito de disco homoclínico. Precisaremos algumas definições preliminares.

Definição 3.1.1. Um disco homoclínico é um disco topológico $D \subset \mathbb{R}^{2}$ limitado por dois segmentos $C$ e $E$ tais que:

(i) $C \subset W^{s}\left(0^{\infty}\right)$ e $E \subset W^{u}\left(0^{\infty}\right)$, onde $W^{s}\left(0^{\infty}\right)$ e $W^{u}\left(0^{\infty}\right)$ são as variedades estáveis e instáveis do ponto $0^{\infty}$;

(ii) existe um conjunto aberto simplesmente conexo $G$ com $\bar{G} \subset D$ tal que $D \cap \Omega(H) \subset G$

(iii) $D \cap H(D)=\emptyset$. 
Observação 3.1.1. Observe que estas condições garantem que as variedades estáveis e instáveis de todos os pontos de $\Omega(H)$ não se acumulam internamente em $\partial D$.

No que segue, um ponto homoclínico será um ponto homoclínico ao ponto $0^{\infty}$ e segmentos de variedades estável e instável serão segmentos de $W^{u}\left(0^{\infty}\right)$ e $W^{s}\left(0^{\infty}\right)$.

Dados os pontos homoclínicos $p_{1}, p_{2}, \ldots, p_{k}$, construa o arco poligonal fechado que consiste da união do segmento de variedade instável unindo $p_{1}$ e $p_{2}$, o segmento de variedade estável unindo $p_{2}$ e $p_{3}$, e assim sucesivamente até o segmento de variedade estável unindo $p_{k}$ e $p_{1}$. Se este arco não tem autointerseções, a região poligonal limitada por ele e denotada por $G\left(p_{1}, . ., p_{k}\right)$.

Para clarificar as seguintes definições veja uma representação esquemática na figura 3.1. As linhas verticais representam as variedades estáveis e as horizontais as variedades instáveis.

Seja $p_{1}=y \cdot x$ um ponto homoclínico. Então seja $N \in \mathbb{N}$ tal que $x=$ $s_{0} s_{1} \ldots s_{N} 10^{\infty}$. Suponha que $\sum_{i=0}^{N} s_{i}+1$ é impar, e que $T \in \mathbb{N}$ tal que $y=$ $0^{\infty} 1 s_{-T} \ldots . . s_{-2} 0$ e que $\sum_{i=1}^{T} s_{-i}+1$ é impar. Definamos $y^{\prime}:=0^{\infty} 1 s_{-T} \ldots s_{-2} 1$ ao qual chamamos o simétrico de $y$. Seja $p_{0}:=y^{\prime} \cdot x$. Note que $p_{0}$ é simétrico a $p_{1}$ em relação a uma horizontal traçada no meio do quadrado que contem a ferradura.

Com estas notações o disco homoclínico $D$ definido por $p_{0}$ e $p_{1}$ é a região $G\left(p_{0}, p_{1}\right)$.

A região instável $L_{D}$ de $D$, a qual denotamos por $L$ quando não ha risco de confusão, é a região $G\left(p_{0}, q_{0}, q_{1}, p_{1}\right) \subset D$ tal que $q_{0}:=y^{\prime} \cdot x^{*}, q_{1}:=y \cdot x^{*}$, onde $x^{*}=s_{0} s_{1} \ldots \overline{s_{N}} 10^{\infty}$ e $\overline{s_{i}}=1-s_{i}$, isto é $x^{*}$ é o sucessor imediato de $x$ na ordem unimodal. Observe que em $L$ não existem pontos do conjunto não-errante da ferradura. Mais ainda, pelo teorema 2.2.3, todo ponto de $L$ tem órbita ilimitada sob iterados futuros de $H$. Por iterados passados, todo ponto de $L$ ou vai para $\infty$ ou pertence à variedade instável de um ponto $z_{1}$ de $\Omega(H)$.

Para $H^{-1}$ fazemos definições similares usando discos homoclínicos que são simétricos em relação a uma vertical traçada no meio do quadrado que contém a ferradura. Assim, um disco homoclínico para $H^{-1}$ é um como o disco $H^{-1}(D)$ na figura 3.1. De maneira similar, definimos $L_{H^{-1}(D)}$ em $H^{-1}(D)$ para $H^{-1}$.

Definição 3.1.2. Um disco homoclínico $D$ é livre para $H$ se

$$
H^{n}(L) \cap D=\emptyset, \forall n \geq 1
$$

Veja figura (3.1) para um exemplo. Similarmente, $H^{-1}(D)$ é livre para $H^{-1}$ se

$$
H^{-n}\left(L_{H^{-1}(D)}\right) \cap H^{-1}(D)=\emptyset, \forall n \geq 1 .
$$

Definição 3.1.3. Um disco homoclínico é um disco de poda se $D$ é livre por $H$ e $H^{-1}(D)$ é livre por $H^{-1}$. O conjunto aberto $\mathbb{P}:=\cup_{i=-\infty}^{\infty} H^{i}(D)$ é chamado a frente de poda associada ao disco $D$. O conjunto $\mathbb{P}_{s}:=h(\mathbb{P} \cap \Omega(H))$ é chamado a frente de poda simbólica. 


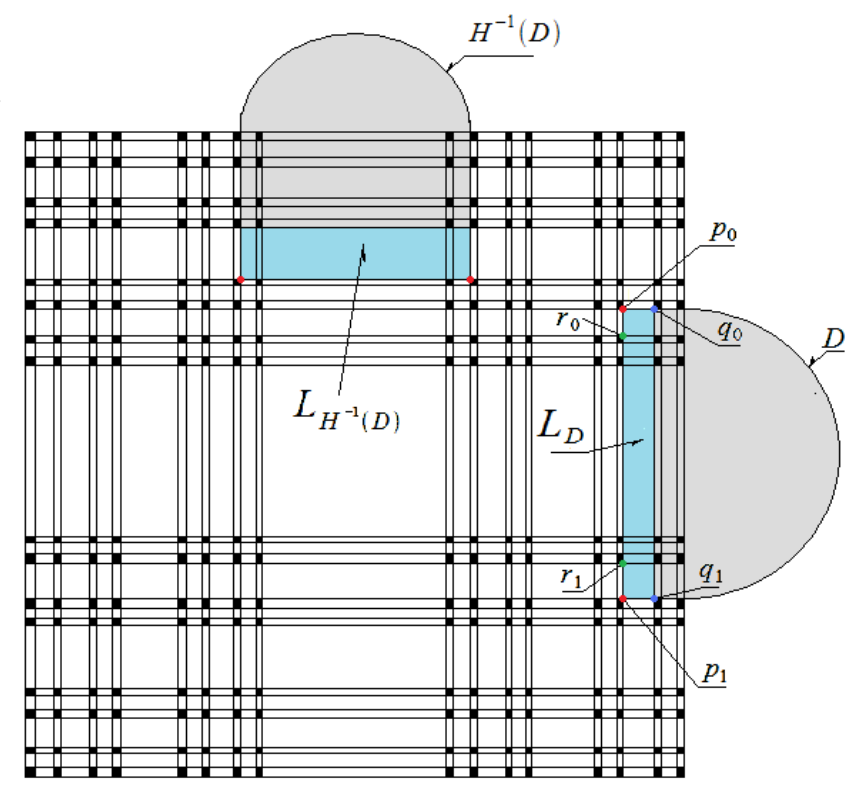

Figura 3.1: Disco homoclínico $D$ definido pelos pontos homoclínicos $p_{0}=0^{\infty} 1111$. $10110^{\infty}$ e $p_{1}=0^{\infty} 1110 \cdot 10110^{\infty}$. Também temos desenhado os principais elementos de um disco de poda. Os discos $D$ e $H^{-1}(D)$ são livres.

Seja $U$ a região $G\left(p_{0}, p_{1}, r_{1}, r_{0}\right)$ onde $r_{0}:=0^{\infty} 1 \overline{s_{-T}} \ldots . . s_{-2} 1 \cdot x$ e $r_{1}:=$ $0^{\infty} 1 \overline{s_{-T}} \ldots . s_{-2} 0 \cdot x$ (Veja a figura 3.1 ). Uma consequência desta definição é a seguinte

Proposição 3.1.1. $L_{H^{-1}(D)}=H^{-1}(U)$.

Demonstração. Note que

$$
\begin{gathered}
H^{-1}\left(p_{0}\right)=0^{\infty} 1 s_{-T} \ldots . . s_{-2} \cdot 1 x, \\
H^{-1}\left(r_{0}\right)=0^{\infty} 1 \overline{s_{-T}} \ldots . . s_{-2} \cdot 1 x, \\
H^{-1}\left(p_{1}\right)=0^{\infty} 1 s_{-T} \ldots . s_{-2} \cdot 0 x, \mathrm{e}, \\
H^{-1}\left(r_{1}\right)=0^{\infty} 1 \overline{s_{-T}} \ldots . . s_{-2} \cdot 0 x .
\end{gathered}
$$

A igualdade segue pela definição de região instável de $H^{-1}(D)$ para $H^{-1}$.

\subsubsection{Exemplos de discos homoclínicos}

Nesta seção apresentamos alguns discos homoclínicos. Em todos os exemplos, representaremos todos estes discos no plano simbólico. Pontos da órbita homoclínica de $p_{0}$ serão representados por pequenos quadrados. Observe que é suficiente fazer um número finito de iterações do disco homoclínico para decidir se é de poda ou não. 
Exemplo 3.1.1. Seja $D_{1}$ o disco homoclínico definido por $p_{0}=0^{\infty} 1111$. $10110^{\infty}$ e $p_{1}=0^{\infty} 1110 \cdot 10110^{\infty}$. Então, $L$ é limitada pelos arcos de variedades definidos por $p_{0}, p_{1}, q_{0}=0^{\infty} 1111 \cdot 10010^{\infty}$ e $q_{1}=0^{\infty} 1110 \cdot 10010^{\infty}$. Neste caso $D_{1}$ é disco de poda. Na figura 3.2(a) mostramos este disco e alguns de seus iterados no plano simbólico.

Exemplo 3.1.2. Seja $D_{2}$ o disco homoclínico definido por $p_{0}=0^{\infty} 1111$. $11010^{\infty}$ e $p_{1}=0^{\infty} 1110 \cdot 11010^{\infty}$. $D_{2}$ é um disco livre, mas $H^{-1}(D)$ não é livre para $H^{-1}$ pois um iterado de $p_{0}$ por $H^{-1}$ está em $D_{2}$. Veja a figura 3.2(b).

Exemplo 3.1.3. As regiões podadas no exemplo da figura 1.2(b) correspondem ao disco homoclínico $D_{3}$ definido pelos pontos homoclínicos $p_{0}=0^{\infty} 11011$. $10110^{\infty}$ e $p_{1}=0^{\infty} 11010 \cdot 10110^{\infty}$. Este é o disco homoclínico conjecturado para a transformação de Hénon $H_{5.4,1}$. Veremos mais tarde que esta conjectura pode ser demonstrada. Neste caso $D_{3}$ é um disco de poda. Veja a figura 3.2(c).

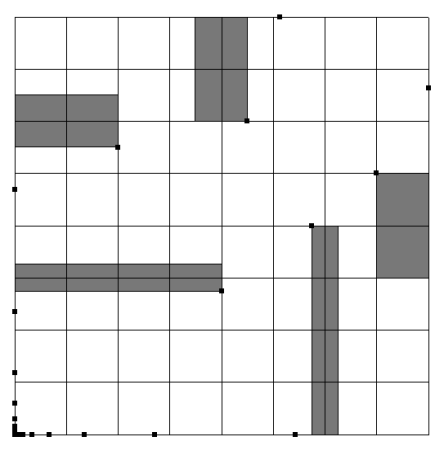

(a) O disco de poda $D_{1}$.

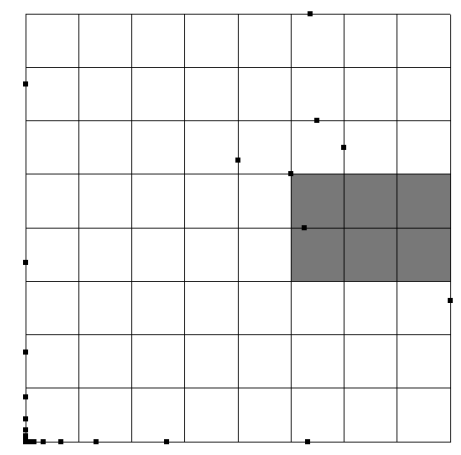

(b) $D_{2}$ não é um disco de poda.

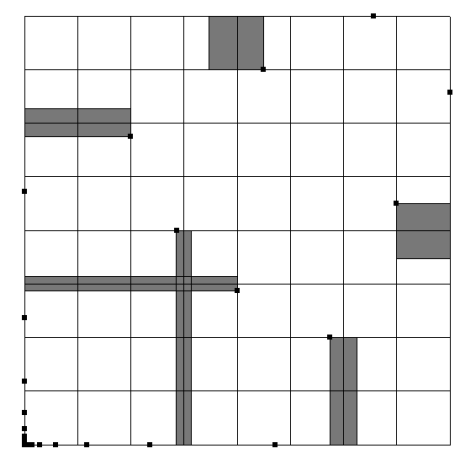

(c) $D_{3}$ é um disco de poda.

Figura 3.2: Exemplos de discos homoclínicos

Uma maneira de construir discos de poda é utilizando a noção de simetria. Definamos primeiro a função $\phi: \Sigma_{2} \rightarrow \Sigma_{2}$ por $(\phi(s))_{i}=s_{-(i+1)}$. Note que $\phi$ 
satisfaz

$$
\phi \circ \sigma \circ \phi=\sigma^{-1}, \mathrm{e}, \phi^{2}=\mathrm{Id} .
$$

Se $A=G\left(p_{0}, p_{1}, . ., p_{k}\right)$ então definamos $\phi(A)$ por $G\left(\phi\left(p_{0}\right), \phi\left(p_{1}\right), \ldots, \phi\left(p_{k}\right)\right)$ onde começamos utilizando o segmento de variedade estável para formar o caminho poligonal.

Um disco $D=G\left(p_{0}, p_{1}\right)$ é simétrico se, e somente se, $\sigma\left(\phi\left(p_{i}\right)\right)=p_{i}$ para $i=0,1$. Isto é equivalente a dizer que $H(\phi(D))=D$ ou $\phi(D)=H^{-1}(D)$.

Proposição 3.1.2. Se D é homoclínico livre e simétrico, então D é um disco de poda.

Demonstração. Observe que $\phi \circ \sigma^{n} \circ \phi=\sigma^{-n}$. Se $D$ é simétrico então, pela proposição 3.1.1, temos que $\phi(L)=L_{H^{-1}(D)}$ e $L=\phi\left(L_{H^{-1}(D)}\right)$. Note que $H^{-n}\left(L_{H^{-1}(D)}\right)$ é uma região limitada segmentos de variedades estáveis e instáveis que passam pelos pontos

$$
\begin{aligned}
& \sigma^{-n}\left(\phi\left(p_{0}\right)\right)=\phi \circ \sigma^{n} \circ \phi\left(\phi\left(p_{0}\right)\right)=\phi \circ \sigma^{n}\left(p_{0}\right), \\
& \sigma^{-n}\left(\phi\left(p_{1}\right)\right)=\phi \circ \sigma^{n} \circ \phi\left(\phi\left(p_{1}\right)\right)=\phi \circ \sigma^{n}\left(p_{1}\right), \\
& \sigma^{-n}\left(\phi\left(q_{0}\right)\right)=\phi \circ \sigma^{n} \circ \phi\left(\phi\left(q_{0}\right)\right)=\phi \circ \sigma^{n}\left(q_{0}\right), \\
& \sigma^{-n}\left(\phi\left(q_{1}\right)\right)=\phi \circ \sigma^{n} \circ \phi\left(\phi\left(q_{1}\right)\right)=\phi \circ \sigma^{n}\left(q_{1}\right),
\end{aligned}
$$

Portanto, $H^{-n}\left(L_{H^{-1}(D)}\right)=\phi\left(H^{n}(L)\right)$. Só temos que provar que $\phi\left(H^{n}(L)\right) \cap$ $\phi(D)=\emptyset$. Mas a última igualdade é verdade, dado que por hipótese, $H^{n}(L) \cap$ $D=\emptyset$.

\subsubsection{Construção da isotopia de poda}

Nesta seção construimos a isotopia que remove os pontos não errantes de $H$ que estão em $D$ e portanto na frente de poda $\mathbb{P}$. A idéia principal é levar todo ponto de $\Omega(H) \cap D$ à região instável $L$ a qual é livre ou errante. Mas observe que o fato de $L$ ser errante não é suficiente para destruir a dinâmica em $D$ da maneira que iremos fazer isso nesta seção.

Quando $a \gg 0$, seguindo as proposições de Hoensch [Hoe08, Proposição 2.1 e 2.2], podemos supor que existe $y_{0}, y_{1}$ tais que os segmentos $C$ e $E$ são curvas que podem ser escritas como graficos de funções sobre o eixo $y$

$$
C=\left\{\left(\gamma_{c}(y), y\right): y \in\left[y_{0}, y_{1}\right]\right\} \text { e } E=\left\{\left(\gamma_{e}(y), y\right): y \in\left[y_{0}, y_{1}\right]\right\} .
$$

Embora Hoensch tenha utilizado a expressão $F_{r, b}=(r x(1-x)-b y, x)$ para a família de Hénon, não é dificil ver que $F_{r, b}$ é conjugado a $H_{a, b}$ pela tranformação

$$
h(x, y)=\left(r\left(x-\frac{1}{2}\right), r\left(y-\frac{1}{2}\right)\right), \operatorname{com} a=-\frac{r}{2}-\frac{b r}{2}+\frac{r^{2}}{4}
$$

e por tanto, as afirmações de [Hoe08] são também verdadeiras para $H_{a, b}$.

Tomemos novas curvas $g_{1}(y), g_{2}(y), g_{3}(y)$ e $g_{4}(y)$ que passem através de $p_{0}$ e $p_{1}$ tais que $\gamma_{c}(y)=: g_{4}(y)<g_{3}(y)<g_{2}(y)<g_{1}(y):=\gamma_{e}(y)$ e $\Omega(H) \cap D \subset$ 


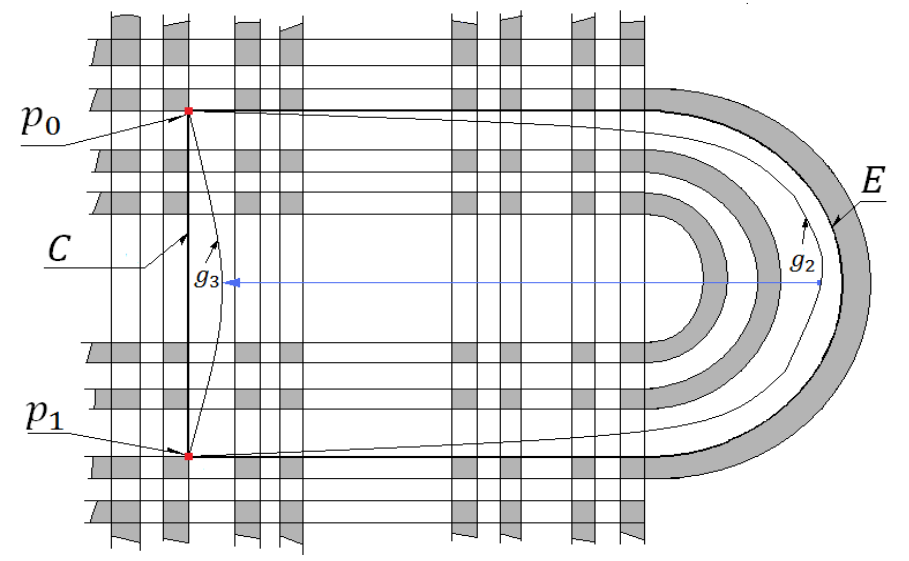

Figura 3.3: Funções $g_{1}, g_{2}, g_{3}$ e $g_{4}$ em um disco de poda homoclínico.

$\left\{(x, y): g_{3}(y)<x<g_{2}(y)\right\}$. Veja a figura (3.3). Por tanto $\left(g_{3}(y), y\right) \in L_{D}$ e $\left(g_{2}(y), y\right) \in U$.

Definição 3.1.4. Seja $g_{t}(y)=g_{2}(y)+t\left(g_{3}(y)-g_{2}(y)\right)$. Definimos a isotopia $S_{t}$ por:

$$
S_{t}(x, y)=\left(f_{t}(x, y), y\right)
$$

onde:

$$
f_{t}(x, y):=\left\{\begin{array}{cc}
\frac{g_{t}(y)-g_{1}(y)}{g_{2}(y)-g_{1}(y)}\left(x-g_{2}(y)\right)+g_{t}(y), & \text { se } g_{2}(y) \leq x \leq g_{1}(y) \\
\frac{g_{4}(y)-g_{t}(y)}{g_{4}(y)-g_{2}(y)}\left(x-g_{4}(y)\right)+g_{4}(y), & \text { se } g_{4}(y) \leq x \leq g_{2}(y) \\
x, & \text { em outro caso. }
\end{array}\right.
$$

Então, $S_{0}=I_{d}$ e $S_{1}\left(g_{2}(y), y\right)=\left(g_{3}(y), y\right), S_{1}\left(g_{1}(y), y\right)=\left(g_{1}(y), y\right), S_{t}\left(g_{4}(y), y\right)=$ $\left(g_{4}(y), y\right)$ e $\operatorname{Supp}\left(S_{t}\right)=\bar{D}$.

Sejam os conjuntos

$$
\begin{aligned}
& A_{1}=\left\{(x, y) / g_{2}(y)<x<g_{1}(y)\right\}, \\
& A_{2}=\left\{(x, y) / g_{3}(y)<x<g_{2}(x)\right\}, \\
& A_{3}=\left\{(x, y) / g_{4}(y)<x<g_{3}(y)\right\} .
\end{aligned}
$$

Definição 3.1.5. A isotopia de poda $H_{t}$ de $H$ é dada por:

$$
H_{t}(x, y):=\left\{\begin{array}{cc}
H \circ S_{t}(x, y) & \text { em } D \\
H(x, y) & \text { em outro caso }
\end{array}\right.
$$


Dado que $D \cap H(D)=\emptyset$ temos que $H_{t}^{-1}$ é bem definida, isto é:

$$
H_{t}^{-1}(x, y):=\left\{\begin{array}{cc}
S_{t}^{-1} \circ H^{-1}(x, y) & \text { em } H(D) \\
H^{-1}(x, y) & \text { em outro caso }
\end{array}\right.
$$

Das definições anteriores se deduzem as proposições:

Proposição 3.1.3. Para um disco homoclínico qualquer temos: $A_{3} \subset L, S_{1}\left(A_{1}\right) \subset$ $A_{1} \cup A_{2}$ e $S_{1}\left(A_{2} \cup A_{3}\right) \subset A_{3}$.

Proposição 3.1.4. A frente de poda para $H$ é o mesmo para $H_{1}$, isto é: $\mathbb{P}=$ $\cup_{i=-\infty}^{\infty} H_{1}^{i}(D)$. Além disso, é invariante por $\mathrm{H}$ e $H_{1}$.

Demonstração. Seja $z \in \mathbb{P}$ e seja $\mathbb{B}:=\cup_{i=-\infty}^{\infty} H_{1}^{i}(D)$. Se $z \in D \cup H(D) \cup$ $H^{-1}(D)=D \cup H_{1}(D) \cup H_{1}^{-1}(D)$, então $z \in \mathbb{B}$, dado que $H(D)=H_{1}(D)$ e $H_{1}^{-1}(D)=H^{-1}(D)$. Seja $z \in \mathbb{P} \cap\left(D \cup H(D) \cup H^{-1}(D)\right)^{c}$. Então, como $z \in H^{i}(D)$ para algum $i \in \mathbb{Z}$, temos que podem acontecer dois casos:

(i) Se $z \in H^{k_{0}}(D)$ para algum $k_{0}>1$. Então $H^{-k_{0}}(z) \in D$. Defina $k_{0}^{*}:=\min \left\{k \in\left\{2, . ., k_{0}\right\} \mid H^{-k}(z) \in D\right\} . \quad$ Logo $H^{-\left(k_{0}^{*}-1\right)}(z) \in H(D)$. Pela definição de $k_{0}^{*}$ temos que $H^{-k}(z) \notin H(D) \cup D, \forall k \in\left\{0, \ldots, k_{0}^{*}-2\right\}$. Logo $H^{-\left(k_{0}^{*}-1\right)}(z)=H_{1}^{-\left(k_{0}^{*}-1\right)}(z) \in H_{1}(D)$ e portanto $H_{1}^{-k_{0}^{*}}(z) \in D$ e $z \in H_{1}^{k_{0}^{*}}(D)$.

(ii) Se $z \in H^{-k_{0}}(D)$ para algum $k_{0}>1$. Então $H^{k_{0}}(z) \in D$. Defina $k_{0}^{*}:=$ $\min \left\{k \in\left\{2, \ldots, k_{0}\right\} \mid H^{k}(z) \in D\right\}$. Então $H^{k}(z) \notin D, \forall k \in\left\{0, . ., k_{0}^{*}-1\right\}$. Então $H^{k_{0}^{*}}(z)=H_{1}^{k_{0}^{*}}(z) \in D$. Logo $z \in H_{1}^{-k_{0}^{*}}(D)$.

Logo $\mathbb{P} \subset \mathbb{B}$.

Por outro lado, seja $z \in \mathbb{B}$. Então podemos supor que $z \notin D \cap H_{1}(D) \cap$ $H_{1}^{-1}(D)$. Logo, existe um $i \in \mathbb{Z}$ tal que $z \in H_{1}^{i}(D)$. Novamente, temos dois casos:

(i) Se $z \in H_{1}^{k_{0}}(D)$ para algum $k_{0}>1$. Então $H_{1}^{-k_{0}}(z) \in D$. Seja $k_{0}^{*}:=$ $\min \left\{k \in\left\{2, \ldots, k_{0}\right\} \mid H_{1}^{-k}(z) \in D\right\} . \quad$ Logo $H_{1}^{-k}(z) \notin D \cup H_{1}(D), \forall k \in$ $\left\{0, . ., k_{0}^{*}-2\right\}$. Logo $H_{1}^{-\left(k_{0}^{*}-1\right)}(z)=H^{-\left(k_{0}^{*}-1\right)}(z) \in H(D)$. Logo $H^{-k_{0}^{*}}(z) \in$ $D$.

(ii) Se $z \in H_{1}^{-k_{0}}(D)$ para algum $k_{0}>1$. Então $H_{1}^{k_{0}}(z) \in D$. Seja $k_{0}^{*}:=$ $\min \left\{k \in\left\{2, . ., k_{0}\right\} \mid H_{1}^{k}(z) \in D\right\}$. Logo, $H_{1}^{k}(z) \notin D, \forall k \in\left\{0, \ldots, k_{0}^{*}-2\right\}$. Daí, $H_{1}^{k_{0}^{*}}=H^{k_{0}^{*}}(z) \in D$.

Por tanto, $\mathbb{B} \subset \mathbb{P}$. Se $p \in \mathbb{P}$ então $p \in H^{i_{0}}(D)$, para algum $i_{0}$, logo existe um $x \in D$ tal que $z=H_{i_{0}}(x)$. Note que $H^{i_{0}-1}(x) \in \mathbb{P}$. Logo $z \in H(\mathbb{P})$. Se $z \in H(\mathbb{P})$, então existe um $i_{0}$ e um $x \in H^{i_{0}}(D)$ tal que $z=H(x)$ e daí, $z=H^{i_{0}+1}(x) \in \mathbb{P}$. Portanto, $\mathbb{P}=H(\mathbb{P})$. De forma similar provamos que $\mathbb{P}=H^{-1}(\mathbb{P})=H_{1}^{-1}(\mathbb{P})=H_{1}^{-1}(\mathbb{P})$.

Proposição 3.1.5. Se D é um disco de poda, então $\Omega\left(H_{1}\right) \cap D=\emptyset$. 
Demonstração. Como o disco $D$ é livre, se $z \in A_{2} \cup A_{3}$ existe um aberto $V:=$ $S_{1}^{-1}(L)$ tal que $z \in V$ e

$$
H_{1}^{n}(V) \cap V=H^{n}(L) \cap V \subset H^{n}(L) \cap D=\emptyset, \forall n \geq 1 .
$$

Logo $z \notin \Omega\left(H_{1}\right)$. Por tanto podemos supor que $z \in A_{1}$. Escolhemos $V$, uma vizinhança de $z$, tal que $V \subset U$. Então, pela proposição 3.1.1, $H_{1}^{-1}(V)=$ $H^{-1}(V) \subset L_{H^{-1}(D)}$. Seja $z_{1} \in V$. Então $H^{-1}\left(z_{1}\right) \in L_{H^{-1}(D)}$. Considere $n\left(z_{1}\right) \geq 0$ o primer enteiro positivo tal que $H^{-n\left(z_{1}\right)}\left(H^{-1}\left(z_{1}\right)\right) \in H(D)$. Então $H^{-n\left(z_{1}\right)-2}\left(H^{-1}\left(z_{1}\right)\right) \in H^{-1}(D)$ o qual é uma contradição porque $H^{-1}(D)$ é livre por $H^{-1}$. Por tanto $\forall n \geq 0$, temos que $H^{-n}\left(H^{-1}\left(z_{1}\right)\right) \notin H(D)$ o que implica que $H_{1}^{-n-1}\left(z_{1}\right) \notin H_{1}(D)$. Assim, temos provado que $H_{1}^{-n-2}\left(z_{1}\right) \notin D$. Novamente, pela proposição 3.1.1, $H_{1}^{-1}\left(z_{1}\right)=H^{-1}\left(z_{1}\right) \notin D$. Logo $H_{1}^{-n}\left(z_{1}\right) \notin$ $D, \forall n \geq 1$. Como o ponto $z_{1}$ foi arbitrario, concluimos que $\forall n \geq 1, H_{1}^{-n}(V) \cap$ $D=\emptyset$ o qual implica que $z \notin \Omega\left(H_{1}^{-1}\right)$, mas sabemos que $\Omega\left(H_{1}\right)=\Omega\left(H_{1}^{-1}\right)$. Por tanto $z \notin \Omega\left(H_{1}\right)$.

Corolário 3.1.1. Se D é um disco de poda, então $\Omega\left(H_{1}\right) \cap \mathbb{P}=\emptyset$.

Demonstração. Suponha que $p \in \Omega\left(H_{1}\right) \cap \mathbb{P}$ então, pela proposição 3.1.4, existe $k_{0} \in \mathbb{Z}$ tal que: $p \in \Omega\left(H_{1}\right) \cap H_{1}^{k_{0}}(D)$. Dado que $\Omega\left(H_{1}\right)$ é $H_{1}$-invariante ou $H_{1}^{-1}$-invariante temos que $H_{1}^{-k_{0}}(p) \in \Omega\left(H_{1}\right) \cap D$, isto é uma contradição com a proposição 3.1.5.

Definição 3.1.6. Seja $\Lambda\left(H_{1}\right):=\Omega(H) \backslash \mathbb{P}$. Observe que $\Lambda\left(H_{1}\right)$ é fechado, $H$-invariante e $H_{1}$-invariante.

Resumindo temos o seguinte lema:

Lema 3.1.1. Se D é um disco de poda então $H_{1}$ é uma poda de $H$, isto é:

(i) $\Omega\left(H_{1}\right) \subset \Lambda\left(H_{1}\right)$,

(ii) $\left.H_{1}\right|_{\Lambda\left(H_{1}\right)}=H$.

Demonstração. Pela construção, $\operatorname{Supp}\left(S_{t}\right)=\bar{D}$ e $\Lambda\left(H_{1}\right) \cap D=\emptyset$. Note que $\left.S_{t}\right|_{\partial D}=I_{d}, \forall t \in[0,1]$. Daí, (ii) é válido. Se $z \in \mathbb{P}$, então, pelo corolário 3.1.1, temos que $z \notin \Omega\left(H_{1}\right)$. Se $z \in(\Omega(H))^{c} \backslash \mathbb{P}$ então $H^{k}(z)=H_{1}^{k}(z), \forall k \in \mathbb{Z}$. Assim, dado que $\Omega(H)$ se pode caracterizar como o conjunto de órbitas limitadas (Teorema 2.2.3), $z \notin K_{a, b}$ e, por tanto, pode acontecer dois casos: (a) Existe uma sequência $H^{n_{i}}(z)$ tal que $\lim _{i \rightarrow \infty}\left|H_{1}^{n_{i}}(z)\right|=\infty$. Neste caso consideramos um número real $R$ e um natural $i_{0}$ tal que $p:=H_{1}^{n_{i_{0}}}(z)=H^{n_{i_{0}}}(z) \in\left(V_{R} \cup D\right)^{c}$, onde

$$
V_{R}=\{(x, y): \max \{|x|,|y|\}<R\} .
$$

Usando um procedimento similar ao usado na prova da proposição 2.2.2, podemos mostrar que $p \notin \Omega\left(H_{1}\right)$ e, então, $z \notin \Omega\left(H_{1}\right)$. (b) Existe uma sequência $H^{-n_{i}}(z)$ tal que $\lim _{i \rightarrow \infty}\left|H_{1}^{-n_{i}}(z)\right|=\infty$ e continuamos como no caso (a). Nos dois casos $z \notin \Omega\left(H_{1}\right)$. Logo, $\Omega\left(H_{1}\right) \subset \Omega(H) \backslash \mathbb{P}$. 
Definição 3.1.7. A transformação $H_{1}$ é chamada o homeomorfismo de poda de $H$ associado ao disco de poda $D$.

Lema 3.1.2. Com as mesmas hipóteses do lema 3.1.1, se $D_{s}=h(D \cap \Omega(H))$ e $\mathbb{P}_{s}:=\cup_{k=-\infty}^{\infty} \sigma^{k}\left(D_{s}\right)$ a frente de poda simbólico, então $h_{s}:=\left.h\right|_{\Lambda\left(H_{1}\right)}$ é uma conjugaçãa entre $\left.H_{1}\right|_{\Lambda\left(H_{1}\right)}$ e $\left.\sigma\right|_{\Sigma_{2} \backslash \mathbb{P}_{s}}$.

Demonstração. Dado que $h$ é um homeomorfismo, então $D_{s}$ é aberto e por tanto $\Sigma_{2} \backslash \mathbb{P}_{s}$ é fechado. Note que por definição, $h_{s}=h: \Lambda\left(H_{1}\right) \rightarrow\left(\Sigma_{2} \backslash \mathbb{P}_{s}\right)$ é bijetora. Observe que $\Lambda\left(H_{1}\right)$ é compacto e $h_{s}$ é contínua. Logo, $h_{s}$ é um homeomorfismo.

\subsubsection{Construção de uma isotopia $C^{\infty}$}

Nesta seção mostraremos a construção da $C^{\infty}$-difeotopia de poda. Também mostramos que neste caso existem variedades estáveis e instáveis locais em todo ponto de $\Lambda\left(H_{1}\right)$ e que em consequência o difeomorfismo de poda $H_{1}$ é hiperbólico.

Lembremos que as curvas que limitam o disco de poda $D$ são variedades invariantes dadas pelas expressões

$$
C:=\left\{\left(\gamma_{c}(y), y\right): y \in\left[y_{0}, y_{1}\right]\right\} \text { and } E:=\left\{\left(\gamma_{e}(y), y\right): y \in\left[y_{0}, y_{1}\right]\right\}
$$

onde $\gamma_{1}(y), \gamma_{2}(y) \in C^{\infty}$ dado que $H_{a, b}$ é um difeomorfismo $C^{\infty}$. Veja o teorema 2.1.1. Logo podemos escolher um $\epsilon_{0}$ tal que as curvas

$$
\alpha:=\left\{\left(\alpha_{1}(y), y\right): y \in\left[y_{0}, y_{1}\right]\right\}=\left\{\left(\gamma_{c}(y)+\epsilon_{0}, y\right): y \in\left[y_{0}, y_{1}\right]\right\}
$$

$\mathrm{e}$

$$
\beta:=\left\{\left(\beta_{1}(y), y\right): y \in\left[y_{0}, y_{1}\right]\right\}=\left\{\left(\gamma_{e}(y)-\epsilon_{0}, y\right): y \in\left[y_{0}, y_{1}\right]\right\}
$$

são bem definidas, se interseptam no interior do disco $D$ e tais que $\alpha \subset L$ e $\beta \subset U$. Sejam $t_{0}^{*}<t_{1}^{*} \in\left(y_{0}, y_{1}\right)$ parâmetros onde $\alpha$ e $\beta$ se intersectam. Definamos

$$
A:=\left\{\left(\gamma_{c}(y)+\epsilon_{0}, y\right), y \in\left[t_{0}^{*}, t_{1}^{*}\right]\right\} \text { e } B:=\left\{\left(\gamma_{e}(y)-\epsilon_{0}, y\right), y \in\left[t_{0}^{*}, t_{1}^{*}\right]\right\}
$$

e seja $K_{1}$ o conjunto compacto limitado por $A$ e $B$. O número $\epsilon_{0}>0$ é escolhido como sendo tal que as interseções das variedades estáveis e instáveis que estão em $D$ ficam contidas em $\operatorname{int}\left(K_{1}\right)$, e os segmentos de variedades estáveis $H$ só intersectam $B$ e os segmentos de variedades instáveis só intersectam $A$. Isto é possível pelas propriedades $i i$ ) e iii) da definição 3.1.1. Então, podemos encontrar um $\epsilon_{1}>0$ tal que

$$
\epsilon_{1}<\min \left\{\operatorname{dist}\left(z, z_{1}\right), z \in K_{1}, z_{1} \in \partial D\right\} .
$$

Dado este $\epsilon_{1}$, definimos os conjuntos:

$$
K_{2}:=\left\{z \in \mathbb{R}^{2}: \operatorname{dist}\left(z, z_{1}\right) \leq \frac{\epsilon_{1}}{4}, \text { para algum } z_{1} \in K_{1}\right\}
$$


$\mathrm{e}$

$$
G_{2}:=\left\{z \in \mathbb{R}^{2}: \operatorname{dist}\left(z, z_{1}\right)<\frac{\epsilon_{1}}{2}, \text { para algum } z_{1} \in K_{1}\right\} .
$$

É claro que $K_{2}$ é compacto, $G_{2}$ é aberto e $K_{1} \subset K_{2} \subset G_{2} \subset D$. Assim, tomamos uma partição da unidade subordinada à cobertura $\left\{G_{2}, \mathbb{R}^{2} \backslash K_{2}\right\}$ de $\mathbb{R}^{2}$. Logo podemos encontrar uma função $\lambda \in C^{\infty}\left(\mathbb{R}^{2}\right)$ tal que $0 \leq \lambda \leq 1, \lambda=1$ em $K_{2}$ e $\operatorname{Supp}(\lambda) \subset G_{2}$. Então definimos o campo vetorial:

$$
X(x, y):=\left\{\begin{array}{cc}
\lambda(x, y)\left(\alpha_{1}(y)-\beta_{1}(y), 0\right) & \text { se }(x, y) \in G_{2} \\
0 & \text { em outro caso }
\end{array}\right.
$$

Pela construção, $X$ é um campo vetorial $C^{\infty}$. Seja $S_{t}(x, y)$ o fluxo gerado por $X(x, y)$.

Proposição 3.1.6. $S_{t} \in C^{\infty}, S_{0}=I_{d}, S_{1}\left(\beta_{1}(y), y\right)=\left(\alpha_{1}(y), y\right)$.

Assim, a difeotopia $C^{\infty}$ é definida por $H_{t}(x, y):=H \circ S_{t}(x, y)$. É fácil ver que, utilizando uma análise similar à da seção 3.1.3, $H_{1}$ satisfaz as conclusões dos lemas 3.1.1 e 3.1.2. Neste caso, a hiperbolicidade de $H$ no conjunto $\Omega(H)$ ajuda-nos a demonstrar a hiperbolicidade de $H_{1}$ em $\Lambda\left(H_{1}\right)$.

Proposição 3.1.7. Existe um $\epsilon_{2}>0$ tal que se $\epsilon<\epsilon_{2}$ e $p \in \Lambda\left(H_{1}\right)$ então: $W_{\epsilon}^{s}\left(p, H_{1}\right)=W_{\epsilon}^{s}(p, H)$ e $W_{\epsilon}^{u}\left(p, H_{1}\right)=W_{\epsilon}^{u}(p, H)$.

Demonstração. Pela construção, se $p \in \Lambda\left(H_{1}\right)$ então $\operatorname{dist}\left(p, \operatorname{Supp}\left(S_{t}\right)\right)>\frac{\epsilon_{1}}{2}$. Tome $\epsilon_{2}<\frac{\epsilon_{1}}{4}$. Seja $\epsilon \leq \epsilon_{2}$ e $z$ tal que $d\left(H_{1}^{n}(p), H_{1}^{n}(z)\right) \leq \epsilon, \forall n \geq 0$. Como $d\left(H_{1}^{n}(p), \operatorname{Supp}\left(S_{t}\right)\right) \geq \epsilon_{2}$ então $d\left(H_{1}^{n}(z), \operatorname{Supp}\left(S_{t}\right)\right)>\epsilon_{2}-\epsilon$. Logo $H_{1}^{n}(z)=$ $H^{n}(z)$. Daí segue que $W_{\epsilon}^{s}\left(p, H_{1}\right)=W_{\epsilon}^{s}(p, H)$. Da mesma maneira provamos que $W_{\epsilon}^{u}\left(p, H_{1}\right)=W_{\epsilon}^{u}(p, H)$.

Assim, provamos o teorema:

Teorema 3.1.1. $H_{1}$ é uma isotopia de poda $C^{\infty}$. Isto é

(i) $\Omega\left(H_{1}\right) \subset \Lambda\left(H_{1}\right)$,

(ii) $\left.H_{1}\right|_{\Lambda\left(H_{1}\right)}=H$ é hiperbólico.

No que segue

toda transformação de poda $H_{1}$ associada a um disco $D$ será um $C^{\infty}$-difeomorfismo hiperbólico em $\Lambda\left(H_{1}\right)$ e chamado difeomorfismo de poda.

\subsection{Shifts de Markov para o difeomorfismo de poda $H_{1}$}

Pela sub-seção 3.1.3 observamos que é possível construir difeomorfismos de poda com discos de poda homoclínicos. Nesta seção provaremos que existe um subshift de tipo finito associado à dinâmica de $H_{1}$ em $\Lambda\left(H_{1}\right)$ e mostramos um algoritmo que permite obter a partição de Markov. 
O algoritmo começa com a partição canônica da ferradura a qual consiste de dois retângulos $R_{0}$ e $R_{1}$. A partir dela construimos a chamada partição de geração-0 para $H_{1}$ que consiste de dois retângulos $B_{(0,1)}$ e $B_{(0,2)}$. Com a partição de geração-0 obtemos uma nova partição, chamada partição de geração 1 , de maneira que o conjunto $\Lambda\left(H_{1}\right)$ ainda esteja contido na união dos elementos da partição. Depois, iteramos a partição para satisfazer as condições da definição 2.1.5. Usando o fato que os pontos que dividem o bordo do disco $D$ são homoclínicos, demonstramos que o algoritmo termina num número finito de passos. Antes, precisamos de algumas definições.

Definição 3.2.1. Se um segmento $\gamma$ da variedade estável de $H$ passa por $D$, então defina o pseudo-segmento de variedade estável de $H_{1}$ por $\gamma_{1}=S_{1}^{-1}(\gamma)$. Se $\gamma$ é um segmento de variedade estável, então o lado estável $l(\gamma)$ que passa por $\gamma$ é a prolongamento de $\gamma$ aos extremos instáveis superior e inferior da ferradura.

Definição 3.2.2. Uma região $L$ está em posição normal na partição $\mathcal{P}$ se, dado um elemento $B \in \mathcal{P}$, ou $L \cap B=\emptyset$, ou $L \subset \operatorname{int}(B)$.

\subsubsection{Partição de geração 1}

Lembre que os pontos que definem o disco de poda são $p_{0}=0^{\infty} 1 s_{-T} \ldots s_{-2} 1$. $s_{0} \ldots s_{N} 10^{\infty}$ e $p_{1}=0^{\infty} 1 s_{-T} \ldots s_{-2} 0 \cdot s_{0} \ldots s_{N} 10^{\infty}$. A partição de geração 1 é construida da seguinte maneira:

- Sejam

$$
R_{0}=G\left(0^{\infty}, 0^{\infty} 1 \cdot 0^{\infty}, 0^{\infty} 1 \cdot 010^{\infty}, 0^{\infty} \cdot 010^{\infty}\right)
$$

e

$$
R_{1}=G\left(0^{\infty} \cdot 110^{\infty}, 0^{\infty} 1 \cdot 110^{\infty}, 0^{\infty} 1 \cdot 10^{\infty}, 0^{\infty} \cdot 10^{\infty}\right)
$$

os elementos da partição canônica da ferradura. Isto é, o retângulo $R_{0}$ corresponde ao símbolo 0 e o retângulo $R_{1}$ corresponde ao símbolo 1 . Então a partição de 0-geração $\mathcal{P}_{0}$ para $H_{1}$ consiste dos conjuntos:

$$
B_{(0,1)}:=S_{1}^{-1}\left(R_{0}\right) \text { and } B_{(0,2)}:=S_{1}^{-1}\left(R_{1}\right)
$$

- Considere o disco $H^{-1}(D)$ e os pontos:

$$
\begin{aligned}
& H^{-1}\left(p_{0}\right)=0^{\infty} 1 s_{-T} . . s_{-2} \cdot 1 s_{0} \ldots s_{N} 10^{\infty} \\
& H^{-1}\left(p_{1}\right)=0^{\infty} 1 s_{-T} . . s_{-2} \cdot 0 s_{0} \ldots s_{N} 10^{\infty} \\
& H^{-1}\left(q_{1}\right)=0^{\infty} 1 s_{-T} . . s_{-2} \cdot 0 s_{0} \ldots \overline{s_{N}} 10^{\infty}
\end{aligned}
$$

$\mathrm{e}$

$$
H^{-1}\left(q_{0}\right)=0^{\infty} 1 s_{-T} . . s_{-2} \cdot 1 s_{0} \ldots \overline{s_{N}} 10^{\infty}
$$

Construa os retângulos limitados pelos pontos:

$$
\begin{aligned}
B_{(1,1)} & :=G\left(0^{\infty}, 0^{\infty} 1 \cdot 0^{\infty}, 0^{\infty} 1 \cdot 0 s_{0} s_{1} \ldots s_{N} 10^{\infty}, 0^{\infty} \cdot 0 s_{0} s_{1} \ldots s_{N} 10^{\infty}\right) \\
B_{(1,2)} & :=G\left(0^{\infty} \cdot 0 s_{0} \ldots \overline{s_{N}} 10^{\infty}, 0^{\infty} 1 s_{-T} . . s_{-2} \cdot 0 s_{0} \ldots \overline{s_{N}} 10^{\infty}, 0^{\infty} 1 s_{-T} . . s_{-2} \cdot 010^{\infty}, 0^{\infty} \cdot 010^{\infty}\right)
\end{aligned}
$$




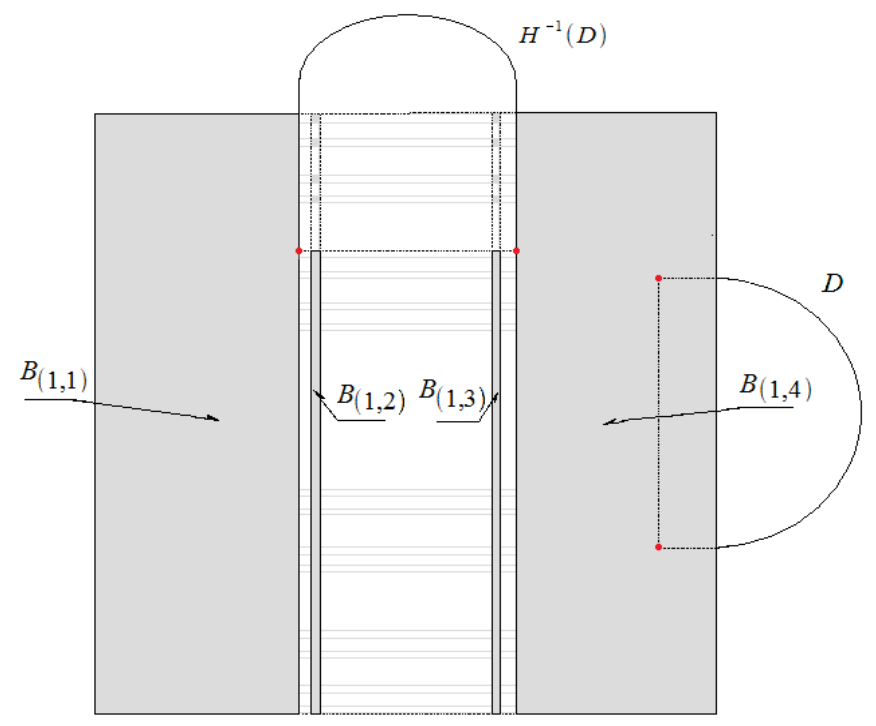

Figura 3.4: Partição de 1-geração para o disco de poda da figura 3.1.

$$
\begin{aligned}
B_{(1,3)} & :=G\left(0^{\infty} \cdot 110^{\infty}, 0^{\infty} 1 s_{-T} . . s_{-2} \cdot 110^{\infty}, 0^{\infty} 1 s_{-T} . . s_{-2} \cdot 1 s_{0} \ldots \overline{s_{N}} 10^{\infty}, 0^{\infty} \cdot 1 s_{0} \ldots \overline{s_{N}} 10^{\infty}\right) \\
B_{(1,4)} & :=G\left(0^{\infty} \cdot 1 s_{0} \ldots s_{N} 10^{\infty}, 0^{\infty} 1 \cdot 1 s_{0} . . s_{N} 10^{\infty}, 0^{\infty} 1 \cdot 10^{\infty}, 0^{\infty} \cdot 10^{\infty}\right)
\end{aligned}
$$

Assim, definimos a partição de 1-geração como sendo

$$
\mathcal{P}_{1}:=\left\{B_{(1.1)}, B_{(1,2)}, B_{(1,3)}, B_{(1,4)}\right\} .
$$

Quando um segmento de variedade estável passa por $D$, use o pseudo-segmento de variedade estável de $H_{1}$. Da construção temos que

$$
\Lambda\left(H_{1}\right) \subset \bigcup_{i=1}^{4} B_{(1, i)}
$$

dado que nenhum ponto de $D$ pertence ao conjunto $\Lambda\left(H_{1}\right)$, e

$$
H_{1}\left(\partial_{s} B_{(1, i)}\right) \cap D=\emptyset, \forall i=1, . ., 4
$$

mas pode acontecer que $H_{1}\left(\partial_{s} B_{(1, i)}\right) \cap H^{-1}(D) \neq \emptyset$, para algum $i$. Por outro lado temos a seguinte:

Proposição 3.2.1. Se D é disco de poda então a região $S_{1}^{-1}\left(L_{D}\right)$ está em posição normal na partição de geração 1 .

Demonstração. A região $S_{1}^{-1}\left(L_{D}\right)$ é limitada por pseudo-segmentos verticais 
de variedade estável e não contêm pontos de $S_{1}^{-1}(\Omega(H))$ e os retângulos da partição de geração 1 são formados por pseudo-segmentos de variedade estável, logo é suficiente mostrar que $L_{D}$ esta em posição normal nos retângulos definidos usando as variedades estáveis de $H$. Suponha que $L_{D} \cap B_{(1, i)} \neq \emptyset$. Se $L_{D}$ contém pontos de $B_{(1, i)}^{c}$ então $L_{D}$ contém um segmento de variedade estável o qual contradiz a definição de $L_{D}$. Se um bordo estável de $L_{D}$ coincide com um bordo estável de $B_{(1, i)}$, então $L_{D}$ têm pontos de $\Omega(H)$ acumulando-se internamente, o que é impossível dado que $L_{D} \cap \Omega(H)=\emptyset$. Logo, os bordos estáveis de $L_{D}$ estão no interior de $B_{(1, i)}$.

\subsubsection{Operações sobre os retângulos}

Note que a partição de geração 1 não satisfaz $H_{1}\left(\partial_{s} B_{i}\right) \subset \bigcup_{j=1}^{l} \partial_{s} B_{j}$. Por outro lado, observe que os lados estáveis de $S_{1}^{-1}\left(L_{D}\right)$ definem uma região que não contém pontos de $S_{1}^{-1}(\Omega(H))$. Para os segmentos estáveis $H_{1}\left(\partial_{s} B_{(1, i)}\right)$ vale que ou pertencem ao lado estável de outro retângulo ou pertencem à um lado estável de $S_{1}^{-1}\left(L_{D}\right)$.

Definição 3.2.3. Seja $B \in \mathcal{P}$ e $L \subset \operatorname{int}(B)$. Então, a operação $P_{1}$ em $B$ é a construção de dois retângulos $B_{1}$ e $B_{2}$ tais que os lados estáveis de $B_{i}$ são os prolongamentos dos lados estáveis de $L$ (Ver figura 3.5(a)).

Definição 3.2.4. Seja $B_{1}, B_{2}, . ., B_{k}$ retângulos de $\mathcal{P}$ e $L^{\prime}$ uma região limitada superiormente por segmentos instáveis, tal que $L^{\prime} \cap \Omega(H)=\emptyset$ tal que $L^{\prime} \cap B_{i} \neq \emptyset$ e os lados estáveis de $L^{\prime}$ estão no interior de $B_{1}$ e $B_{k}$ (a figura 3.5(b) mostra os retângulos con $k=2$ ). Então, a operação $P_{2}$ de $B_{1}, B_{2}, . ., B_{k}$ é a contrução de $k+1$ retângulos, reduzindo o lado instável inferior de cada $B_{i}$ ao lado instável superior de $L^{\prime}$ e definindo $B_{k+1}$ como o retângulo limitado superiormente pelo segmento instável que passa pelo lado instável inferior de $L^{\prime}$, pelos lados estáveis de $B_{1}$ e $B_{k}$ e inferiormente pelo segmento instável inferior (Ver figura 3.5(b)).

Sejam $p$ e $q$ dois pontos em $\Sigma_{2}$. Então a x-pseudo-distância simbólica entre $p$ e $q, d_{x}(p, q)$ é a distância entre as coordenadas $x$, isto é:

$$
d_{x}(p, q):=d\left(0^{\infty} \cdot p_{x}, 0^{\infty} \cdot q_{x}\right)
$$

Observe que quando $p$ e $q$ são pontos homoclínicos ao ponto $0^{\infty}$ que estão no mesmo segmento horizontal e são consecutivos, isto é, $p$ é o sucessor imediato de $q$ na ordem unimodal ou $q$ é o sucessor de $p$, então $\sigma$ duplica sua $x$-pseudodistancia e $\sigma^{-1}$ divide-a por 2 .

\subsubsection{Algoritmo}

a1) Como $S_{1}^{-1}\left(L_{D}\right)$ está em posição normal na partição de geração 1 , então aplicamos a operação $P_{1}$ ao retângulo que contém $S_{1}^{-1}\left(L_{D}\right)$, isto gera a partição de geração 2 .

a2) Iteramos a região $S_{1}^{-1}\left(L_{D}\right)$ por $H_{1}$ ou, que é o mesmo, $L_{D}$ por $H$. Em cada iteração, $H^{k}\left(L_{D}\right), k \geq 0$ está em posição normal na partição de 


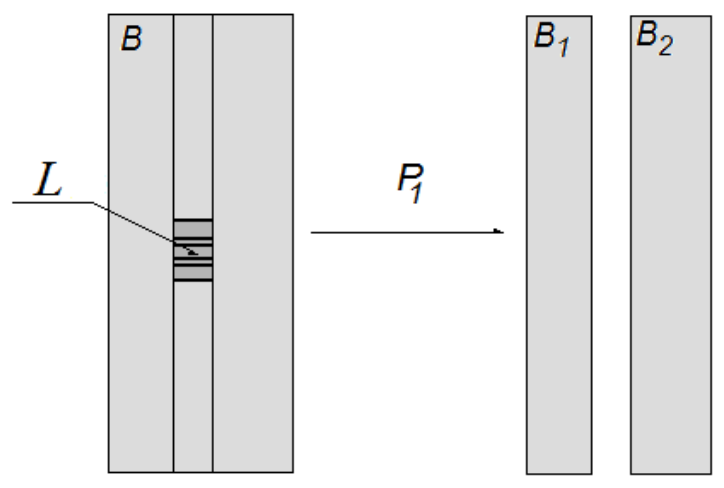

(a) Operação $P_{1}$.

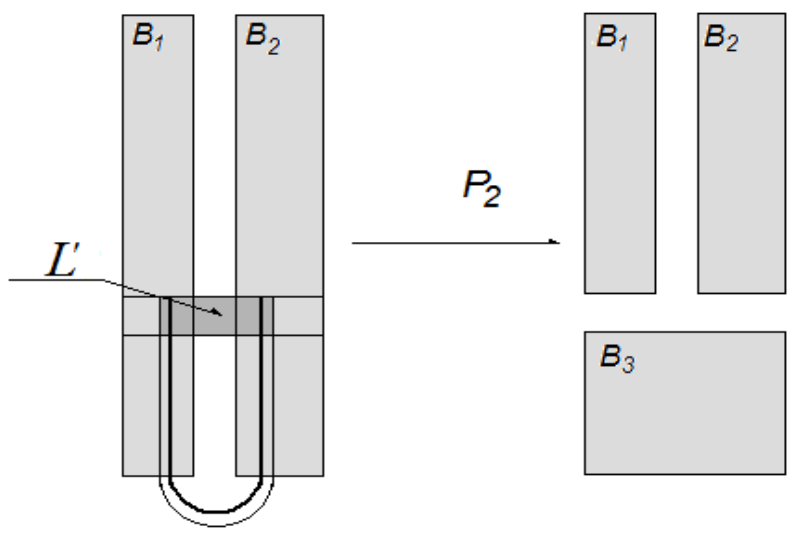

(b) Operação $P_{2}$

Figura 3.5: Descrições pictóricas das operação $P_{1}$ (Ver figura 3.5(a)) e $P_{2}$ (Ver figura $3.5(\mathrm{~b}))$.

geração $k+1$ o qual permite aplicar a operação $P_{1}$ no retângulo que contêm $H^{k}\left(L_{D}\right)$ e obter a partição de geração $k+2$.

Observe que a $x$-pseudo-distância entre os lados estáveis de cada $H_{1}^{k}\left(L_{D}\right)$ cresce por um factor 2 o qual garanta que $H^{k}\left(L_{D}\right)$ está em posição normal na partição de geração $k+1$. Novamente, este processo permite construir partições tais que

$$
H_{1}\left(\partial_{s} B_{(k+2, i)}\right) \cap D=\emptyset, \forall i
$$

e

$$
H_{1}\left(\partial_{s} B_{(k+2, i)}\right) \subset \bigcup_{j=1}^{l} \partial_{s} B_{(k+2, j)} \text { ou } H_{1}\left(\partial_{s} B_{(k+2, i)}\right) \cap H^{-1}(D) \neq \emptyset .
$$


Este processo termina depois de $N-1$ iterados dado que $H^{N}\left(L_{D}\right)$ tem os lados estáveis nos segmentos estáveis que passam pelos pontos $0^{\infty} \cdot s_{N} 10^{\infty}$ e $0^{\infty}$. $\overline{s_{N}} 10^{\infty}$ que já pertencem ao lado estável de algum retângulo $B_{\left(N+1, i_{0}\right)}$. Se pode provar que por este procedimento temos construido $4+N$ retângulos da partição de geração $N+1$ tais que $H\left(\partial_{s} B_{i}\right) \subset \bigcup_{j=1}^{l} \partial_{s} B_{j}$ ou $H\left(\partial_{s} B_{i}\right) \cap H_{1}^{-1}(D) \neq \emptyset$.

Com os retângulos da partição de geração $N+1$, definiremos uma partição que satisfara:

$$
H^{-1}\left(\partial_{u} B_{i}\right) \subset \bigcup_{j=1}^{l} \partial_{u} B_{j}
$$

Além disso, esta nova partição também permitirá descartar a possibilidade que $H\left(\partial_{s} B_{i}\right) \cap H_{1}^{-1}(D) \neq \emptyset$.

Os retângulos da partição de geração $N+1$ cujos bordos instáveis estão no bordo superior da ferradura já satisfazem a condição 3.28. Logo, só temos que utilizar aqueles cujo bordo não chega à parte instável superior da ferradura. Mas, eles são os retângulos cujo bordo superior está no bordo instável de $H^{-1}(D)$. Portanto,

a3) continuamos o processo iterando $H^{-1}(D)$ por $H_{1}^{-1}$ ou, mais precisamente, $L_{H^{-1}(D)}$ por $H_{1}^{-1}$

a4) na iteração $k \geq 1, H_{1}^{-k}\left(L_{H^{-1}(D)}\right)$ satisfaz as condições para aplicar a operação $P_{2}$ na partição de geração $N+k$.

Observe que a $x$-pseudo-distância entre $H_{1}^{-1}\left(p_{1}\right)$ e $H_{1}^{-1}\left(q_{1}\right)$ decresce por iterados negativos de $H_{1}$ num fator 0.5 . Isto permite construir a partição de geração $N+1+k$ que contêm $4+N+k$ retângulos. Observe também que isto elimina a possibilidade que $H_{1}\left(\partial_{s} B_{i}\right) \cap H^{-1}(D) \neq \emptyset$. O processo termina depois de $T-2$ iterados dado que os vértices de $H_{1}^{-(T-2)}\left(L_{H^{-1}(D)}\right)$ estão nos lados instáveis que passam por $y=0^{\infty} 1 \overline{s_{-T}}$ e $y=0^{\infty} 1 s_{-T}$. Portanto temos construido a partição de geração $N+T-1(=N+1+T-2) \operatorname{com} 2+N+T(=4+N+T-2)$ retângulos. Da construção é claro que a condição 3.28 é satisfeita e que:

$$
\Lambda\left(H_{1}\right) \subset \bigcup_{i=1}^{2+N+T} B_{i} .
$$

Exemplo 3.2.1. Se $D$ é o disco de poda da figura 3.1, isto é um disco homoclínico limitado pelos pontos $p_{0}=0^{\infty} 1111 \cdot 10110^{\infty}$ e $p_{1}=0^{\infty} 1110 \cdot 10110^{\infty}$, então a partição de 1-geração é a partição mostrada na figura 3.4. A partição de 2 -geração, $\mathcal{P}_{2}$, é obtida aplicando a operação 1 ao retângulo $B_{(1,4)}$. O resultado está na figura 3.6. Logo, aplicamos novamente a operação 1 a $\mathcal{P}_{2}$ e construimos a partição de 3-geração, $\mathcal{P}_{3}$. Isto está mostrado na figura 3.7. Note que esta partição satisfaz a condição da equação 2.18. Asim, podemos aplicar a operação 2 a região $L_{H^{-1}(D)}$ para obter a última partição de 4-geração, $\mathcal{P}_{4}$, mostrada na figura 3.8. $\mathcal{P}_{4}$ é a partição de Markov procurada. 


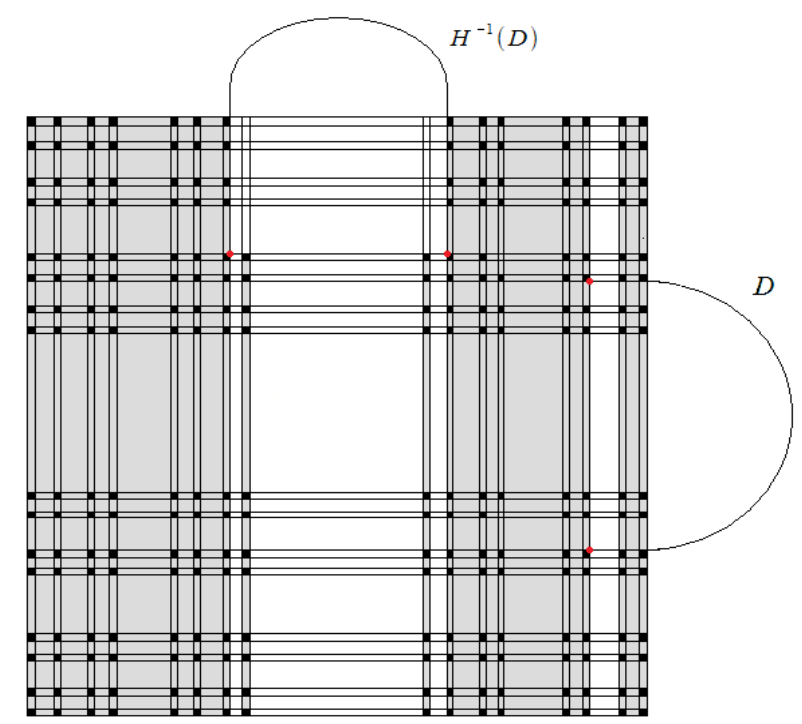

Figura 3.6: Partição de 2-geração para o disco de poda da figura 3.1.

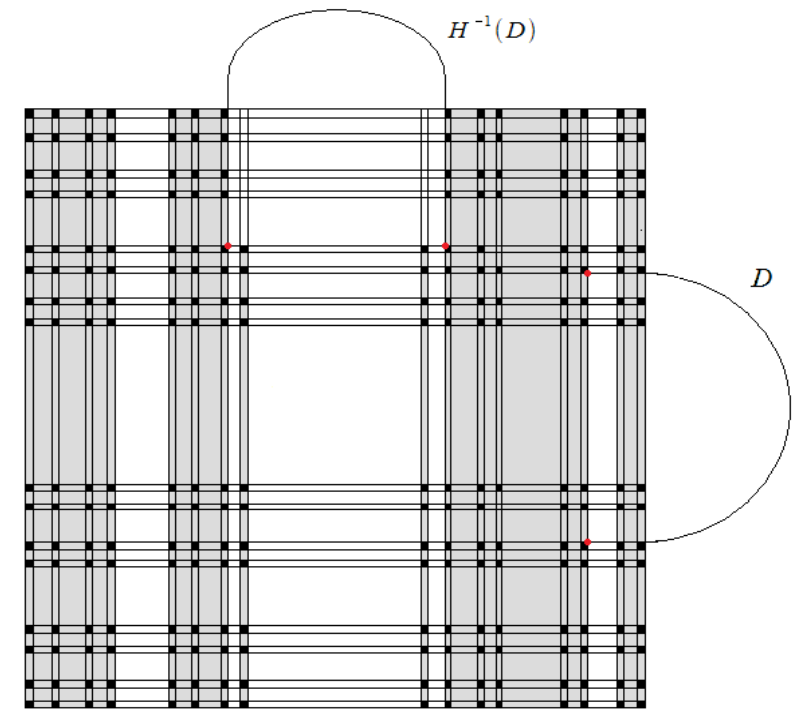

Figura 3.7: Partição de 3-geração para o disco de poda da figura 3.1. 


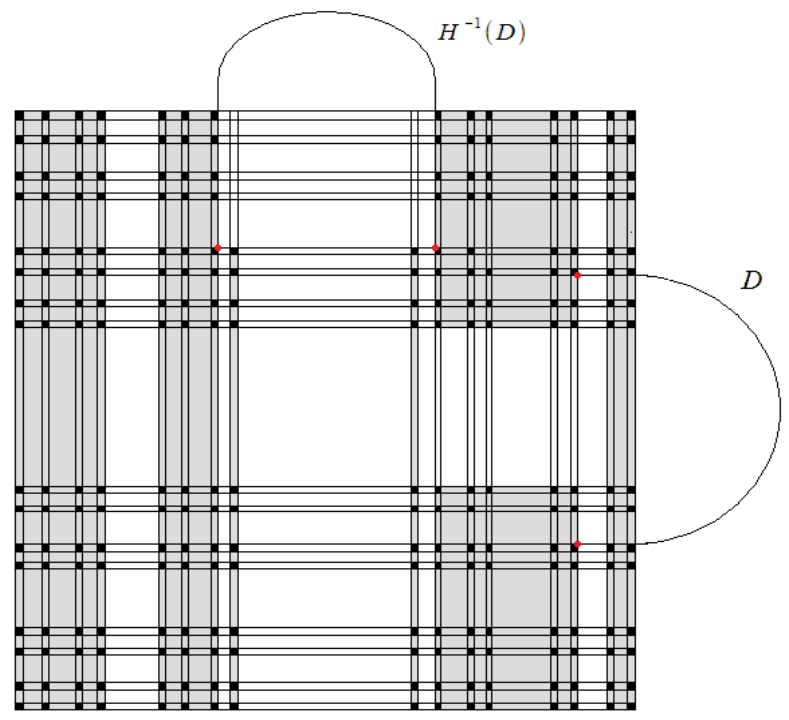

Figura 3.8: Partição de 4-geração para o disco de poda da figura 3.1.

\subsection{Prova do teorema de poda}

Nesta seção demonstramos que existe uma conjugação entre o difeomorfismo $H_{1}$, restrito ao seu conjunto não errante, e $\sigma$ em $\Sigma_{A}$ onde $A$ é a matriz de transição da partição obtida pelo algoritmo anterior. Também, provamos que $\Omega\left(H_{1}\right)=\Lambda\left(H_{1}\right)$.

Seja $\mathcal{P}=\left\{B_{1}, \ldots, B_{2+T+N}\right\}$ a partição de generação $N+T-1$. Se $k:=$ $N+T+2$, defina a matriz $A \in M_{k}$ por: $A_{i j}=1$ se $H_{1}\left(\operatorname{int}\left(B_{i}\right)\right) \cap \operatorname{int}\left(B_{j}\right) \neq \emptyset$ e $A_{i j}=0$ em outro caso. Para cada $z \in \Lambda\left(H_{1}\right)$ podemos associar um elemento $a:=\tau(z) \in \Sigma_{A}$ seguindo o itinerário de $z$ entre os elementos de $\mathcal{P}$, isto é, $\forall i \in \mathbb{Z}, H_{1}^{i}(z) \in B_{a_{i}}$. Com esta definição, o seguinte diagrama comuta:

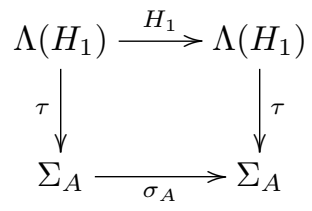

Isto é: $\tau \circ H_{1}=\sigma_{A} \circ \tau$.

A seguinte é uma importante observação.

Observação 3.3.1. Da construção, para um retângulo $B_{i}$ da partição de Markov $\mathcal{P}$ vale que ou $B_{i} \cap \Lambda\left(H_{1}\right) \subset R_{0}$ ou $B_{i} \cap \Lambda\left(H_{1}\right) \subset R_{1}$. Assim, existe um $r$ tal que os índices podem ser organizados $B_{i_{l}} \cap \Lambda\left(H_{1}\right) \subset R_{0}, l=1, . ., r$ e $B_{i_{l}} \cap \Lambda\left(H_{1}\right) \subset R_{1}, l=r+1, . ., k$. 
Definição 3.3.1. Defina $\psi: \Sigma_{A} \rightarrow \Sigma_{2}$ da seguinte maneira: dado $a \in \Sigma_{A}$, $\psi(a) \in \Sigma_{2}$ é tal que $(\psi(a))_{j}=0$ se $a_{j} \in\left\{i_{1}, \ldots, i_{r}\right\}$ e $(\psi(a))_{j}=1$ se $a_{j} \in$ $\left\{i_{r+1}, \ldots, i_{k}\right\}$. Dado $z \in \Lambda\left(H_{1}\right)$ com $\tau(z)=a$, defina $\psi^{*}(z)=\psi(\tau(z))=\psi(a)$. Como $H_{1}=\left.H\right|_{\Lambda\left(H_{1}\right)}$, temos $\psi^{*}(z)=h(z)$ ou $\psi \circ \tau=h_{s}$. Lembre que $h_{s}=$ $\left.h\right|_{\Lambda\left(H_{1}\right)}$.

Teorema 3.3.1 (Teorema de poda). Seja $D$ um disco de poda e $H_{1}$ o difeomorfismo de poda associado. Então $H_{1}$ restrito $\Omega\left(H_{1}\right)$ é topologicamente conjugado ao shift $\sigma_{A}$ no espaço $\Sigma_{A}$ onde $A$ é a matriz definida pelo algoritmo anterior.

Demonstração. A prova consiste dos seguintes passos:

(i) $\tau$ é injetora. Sejam $z_{1}, z_{2} \in \Lambda\left(H_{1}\right)$ tais que $\tau\left(z_{1}\right)=\tau\left(z_{2}\right)$. Pela definição 3.3.1, temos $\psi\left(\tau\left(z_{1}\right)\right)=\psi\left(\tau\left(z_{2}\right)\right)$ e daí, $h_{s}\left(z_{1}\right)=h_{s}\left(z_{2}\right)$. Logo, $z_{1}=z_{2}$.

(ii) $\tau$ é sobrejetora. Seja $a \in \Sigma_{A}$ uma sequência admissível. Considere $\psi(a)$ como na definição 3.3.1. Logo, existe um único $z \in \Omega(H)$ tal que $h(z)=$ $\psi(a)$. Provaremos que $z \in \Lambda\left(H_{1}\right)$ e $\tau(z)=a$. Dado que $\mathcal{P}$ é uma partição de Markov [Shu87] temos que $\mathcal{F}_{n}:=\cap_{s=-n}^{n} H_{1}^{-s}\left(B_{a_{s}}\right)$ é uma sequência decrescente de compactos não vazíos. Logo, a interseção $\mathcal{F}:=\cap_{n=1}^{\infty} \mathcal{F}_{n}$ é não vazía. Seja $z^{*} \in \mathcal{F}$, então $\tau\left(z^{*}\right)=a$. Se $z^{*} \in \mathbb{P}$ então existe um $n_{0} \in \mathbb{Z}$ tal que $H_{1}^{n_{0}}\left(z^{*}\right) \in D$ e pela definição de disco de poda homoclínico temos que $H_{1}^{n_{0}}\left(z^{*}\right)$ tem órbita passada e/ou futura não limitada, isso é uma contradição com a construção de $z^{*}, \operatorname{logo} z^{*} \in \mathbb{P}^{c}$. Portanto $H_{1}^{i}\left(z^{*}\right)=$ $H^{i}\left(z^{*}\right)$ o que implica que $z$ tem órbita limitada para $H$. Assim, pelo teorema 2.2.3, $z^{*} \in \Omega(H)$. Logo $h_{s}\left(z^{*}\right)=\psi\left(\tau\left(z^{*}\right)\right)=\psi(a)=h(z)$ e então $z^{*}=z$. Logo $z \in \Omega(H) \backslash \mathbb{P}=\Lambda\left(H_{1}\right)$.

(iii) $\tau$ é contínua. Note que, de (ii), faz sentido definir a aplicação $\Phi: \Sigma_{A} \rightarrow$ $\Lambda\left(H_{1}\right)$ da forma $\Phi(a):=\cap_{s=-\infty}^{\infty} H_{1}^{-s}\left(B_{a_{s}}\right)$, isto é, $\Phi$ é a inversa de $\tau$ e é injetora e sobrejetora. Seja $a \in \Sigma_{A}, U$ uma vizinhança de $z=\Phi(a)$ em $\Lambda\left(H_{1}\right)$ e $\left\{b^{n}\right\}_{n \in \mathbb{N}}$ uma sequência em $\Sigma_{A}$ que converge à $a$. Seja $\mathcal{F}_{n}$ os compactos definidos como em (ii); dado que a interseção dos $\mathcal{F}_{n}$ é só um ponto e $\mathcal{F}_{n}$ é uma sequência decrescente de compactos, existe um $n_{0}$ tal que para todo $m \geq n_{0}, \mathcal{F}_{m} \subset U$. Como $b^{n}$ converge a $a$, existe um $n_{1}$ tal que:

$$
\forall i \text { com }|i|<n_{0}, \forall m>n_{1} \text { temos que } b_{i}^{m}=a_{i} .
$$

Portanto, quando $m>n_{1}, \Phi\left(b^{m}\right) \in \mathcal{F}_{n_{0}}$, e por tanto esta em $U$. Como $U$ e $\left\{b^{n}\right\}$ são arbitrarios, $\Phi$ é contínua. Dado que $\Sigma_{A}$ é compacto, $\Phi$ é um homeomorfismo.

Pelo lema 3.1.1 temos que $\Omega\left(H_{1}\right) \subset \Lambda\left(H_{1}\right)$. Por (i), (ii) e (iii) temos que todo ponto de $\Lambda\left(H_{1}\right)$ é não errante dado que todo ponto de $\Sigma_{A}$ é não-errante (eles podem ser aproximados por órbitas periódicas). Logo $\Lambda\left(H_{1}\right) \subset \Omega\left(H_{1}\right)$. Portanto $\Omega\left(H_{1}\right)=\Omega(H) \backslash \mathbb{P}$. 


\section{Capítulo 4}

\section{Poda para o derivado da ferradura}

A existência da região livre $L$ num disco homoclínico $D$ é a principal ferramenta para construir podas da ferradura que sejam diferenciáveis. Note que a definição de $L$ depende dos fatos que as órbitas que definem o disco $D$ são órbitas homoclínicas ao ponto $0^{\infty}$ e que as projeções destas sobre o eixo $x$ são os extremos das lacunas de um conjunto de Cantor unidimensional. Isto não é o caso para outras órbitas periódicas pois um ponto periódico pode ser aproximado por órbitas que estão a direita e à esquerda dele.

Neste capítulo construiremos difeomorfismos de poda da ferradura utilizando órbitas homoclínicas a outros pontos periódicos. A ideia da construção é, como no capítulo 3, encontrar regiões livres limitadas por segmentos de variedades invariantes ao ponto periódico; sem embargo, pelo dito no parágrafo anterior, não podemos encontrar estas regiões diretamente na ferradura. Mas, fazendo uma construção, similar a aquelas para derivados de Anosov, podemos abrir o ponto periódico em três pontos tais que para um deles existem discos homoclínicos análogos aos discos homoclínicos associados ao ponto fixo $0^{\infty}$. As transformações obtidas assim serão chamadas derivadas da ferradura. Veja a seção 4.1.

Feito isso, construir transformações de poda com esses discos será equivalente a definir regiões que sejam livres (de maneira anâloga à região instável $L$ do capítulo anterior) ou atratoras que serão definidas na seção 4.2. Assim, nesta mesma seção enunciamos um teorema de poda para o derivado da ferradura.

Então, no final deste capítulo, na seção 4.3, apresentamos um teorema de poda diferenciável que generaliza o teorema de poda para difeomorfismos hiperbólicos em superfícies.

\subsection{Derivados da ferradura}

\subsubsection{Derivados de Anosov}

O primeiro exemplo de um derivado de Anosov foi dado por Smale em [Sma67] fazendo um tipo de cirurgia a um difeomorfismo de Anosov $f$ do toro $\mathbb{T}^{2}$. Esta cirurgia é feita mudando o difeomorfismo de Anosov numa pequena vizinhança quadrada ao redor do ponto fixo correspondente ao ponto $(0,0)$ em $\mathbb{R}^{2}$.

Inicialmente temos que o quadrado $Q=A B C D$ é levado linearmente por 
$f$ em $A^{\prime} B^{\prime} C^{\prime} D^{\prime}$ como na figura 6.2(a). Sem mudar o difeomorfismo fora de $Q$, podemos mudar $f$ tal que temos três pontos fixos em $Q$ como é ilustrado na figura 6.2(b): um ponto atrator $x$ e dois pontos hiperbólicos.

Assim, $\mathbb{T}^{2}$ pode ser escrito como a união de uma variedade estável bidimensional do ponto $x, W^{s}(x)$, e um conjunto unidimensional $f$-invariante $\Lambda$. Escolhendo convenientemente a deformação, podemos fazer que $\Lambda$ seja um conjunto hiperbólico.

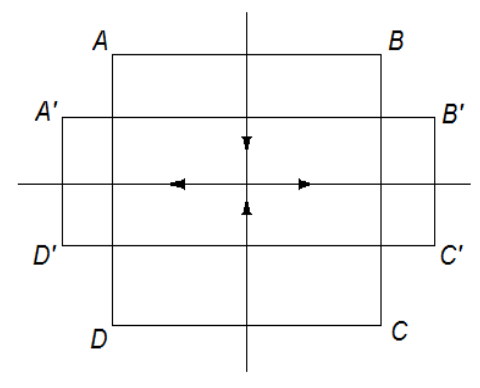

(a) Vizinhança do ponto fixo.

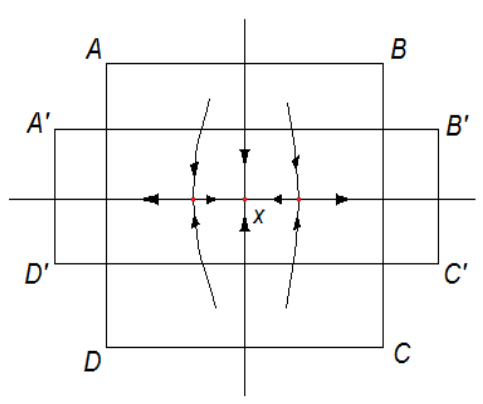

(b) Deformação em $Q$.

Figura 4.1: Ponto fixo do difeomorfismo de Anosov $f$ (Figura 6.2(a)) e o derivado de Anosov (Figura 6.2(b)).

\subsubsection{Cirugia num ponto periódico}

Nesta seção faremos uma deformação similar à anterior em pontos periódicos da ferradura. Seguiremos de perto a construção de derivados de Anosov de [KH95, Seção 17.2].

Considere uma ferradura $F$ tal que o seguinte se satisfaz:

A1. $F$ é uma ferradura hiperbólica no plano de classe $C^{\infty}$.

A2. As variedades estáveis associadas a todos os pontos de $\Omega(F)$ são verticais e que as variedades instáveis são horizontais.

A3. A codificação simbólica em $\{0,1\}^{\mathbb{Z}}$ de um ponto $(x, y) \in \Omega(F)$ é dada como na seção 2.1.3, isto é:

$$
(h(x, y))_{i}= \begin{cases}0 & \text { se } \pi_{1}\left(F^{i}(x, y)\right)<0 \\ 1 & \text { se } \pi_{1}\left(F^{i}(x, y)\right)>0\end{cases}
$$

A4. A partição canônica de $F$ consiste de dois retângulos:

$$
R_{0}=G\left(0^{\infty}, 0^{\infty} 1 \cdot 0^{\infty}, 0^{\infty} 1 \cdot 010^{\infty}, 0^{\infty} \cdot 010^{\infty}\right)
$$


e

$$
R_{1}=G\left(0^{\infty} \cdot 110^{\infty}, 0^{\infty} 1 \cdot 110^{\infty}, 0^{\infty} 1 \cdot 10^{\infty}, 0^{\infty} \cdot 10^{\infty}\right) .
$$

onde $R_{0}$ corresponde ao símbolo 0 e $R_{1}$ corresponde ao símbolo 1 .

A5. O conjunto não-errante $\Omega(F)$ é igual ao conjunto de órbitas limitadas:

$$
K=\left\{(x, y) \in \mathbb{R}^{2}:\left\{F^{n}(x, y)\right\}_{n \in \mathbb{Z}} \text { é limitada }\right\} .
$$

Uma órbita periódica $P$ de período $N$ será descrita pelo seu codigo simbólico $c_{P} \in\{0,1\}^{N}$ o qual é dado pelos primeiros $N$ símbolos do itinerário do seu ponto que fica mais à direita $p$, assim:

$$
\tau(p)=c_{P}^{\infty} \cdot c_{P}^{\infty}
$$

Doravante $c_{P}=s_{1} s_{2} \ldots s_{N}$. Da definição se $c_{P} \neq 0, s_{1}=1$. Defina os números $r_{P}(i)=\sum_{j=1}^{i} s_{j}$, ou seja, o número de 1 's que existem até os primeiros $i$ símbolos. Quando seja claro qual é a órbita periódica estudada, simplesmente escreveremos $r(i)$ em vez de $r_{P}(i)$.

Proposição 4.1.1. Seja $P$ uma órbita periódica de período $N$ então:

i) $S e r_{P}(N)$ é par então $D F^{N}(p)$ tem autovalores positivos.

ii) $\operatorname{Se}_{P}(N)$ é impar então $D F^{N}(p)$ tem autovalores negativos.

Demonstração. Segue dos fatos que:

- Se $(x, y) \in \Omega(H) \cap R_{0}$ então a derivada $D F(x, y)$ tem autovalores positivos,

- Se $(x, y) \in \Omega(H) \cap R_{1}$ então a derivada $D F(x, y)$ tem autovalores negativos.

Para $\epsilon>0$ muito pequeno, considere um disco $U$ centrado em $p$ e de raio $\epsilon$. Tome $\epsilon$ tão pequeno que em $U$ não existam outros pontos periódicos de período $N$ nem pontos de período $2 N$, e tal que $f^{i}(U) \cap U=\emptyset, \forall i=\{1, . ., N-1\}$. Isto é possível pois na ferradura só existe um número finito de pontos periódicos de um período dado. Em $U$ introduzimos coordenadas locais $\left(x_{1}, x_{2}\right)$ tal que

$$
F^{N}\left(p+\left(x_{1}, x_{2}\right)\right)=p+\left(\lambda x_{1}, \mu x_{2}\right)
$$

onde $\lambda$ e $\mu$ são os autovalores de $D F^{N}(p) \operatorname{com}|\lambda|>1$ e $0<|\mu|<1$.

Defina $\lambda_{1}=\sqrt[N]{|\lambda|}$ e $\mu_{1}=\sqrt[N]{|\mu|}$. Assim $\lambda=(-1)^{r(N)} \lambda_{1}^{N}$ e $\mu=(-1)^{r(N)} \mu_{1}^{N}$.

Sem perda de generalidade, podemos supor que em $U$, o $i$-iterado da ferradura $F$ é dado por

$$
F^{i}\left(p+\left(x_{1}, x_{2}\right)\right)=F^{i}(p)+(-1)^{r(i)}\left(\lambda_{1}^{i} x_{1}, \mu_{1}^{i} x_{2}\right) .
$$


Para construir um derivado da ferradura, primeiro definimos, dado $\epsilon>0$, uma $C^{\infty}$-função $\phi: \mathbb{R} \rightarrow[0,1]$ tal que

$$
\begin{gathered}
\phi(-t)=\phi(t), \quad(t \in \mathbb{R}), \\
\phi(t)= \begin{cases}1 & \text { se }|t| \leq \epsilon / 8, \\
0 & \text { se }|t| \geq \epsilon / 4,\end{cases} \\
\phi^{\prime}(t)<0, \text { se } \epsilon / 8<t<\epsilon / 4 .
\end{gathered}
$$

Agora tomemos $k \in \mathbb{R}$ suficientemente grande (e que será especificado posteriormente) e definamos $f: \mathbb{R}^{2} \rightarrow \mathbb{R}^{2}$ por

$$
\left.f\right|_{\mathbb{R}^{2} \backslash U}=\left.F\right|_{\mathbb{R}^{2} \backslash U}
$$

e

$$
f\left(p+\left(x_{1}, x_{2}\right)\right)=F\left(p+\left(x_{1}, x_{2}\right)\right)+(-1)^{r(1)}\left(m \phi\left(k x_{1}\right) \phi\left(x_{2}\right) x_{1}, 0\right), \text { em } U .
$$

onde $m$ é um número real a ser definido. O difeomorfismo $f$ será chamado derivado da ferradura (DF).

Assim, como na equação 4.7, temos

$$
f^{i}\left(p+\left(x_{1}, x_{2}\right)\right)=F^{i}(p)+(-1)^{r(i)}\left(\lambda_{1}^{i} x_{1}+\lambda_{1}^{i-1} m \phi\left(k x_{1}\right) \phi\left(x_{2}\right) x_{1}, \mu_{1}^{i} x_{2}\right)
$$

Por tanto as derivadas de $f$ e $f^{N}$ em $U$ são:

$$
\begin{gathered}
D f\left(p+\left(x_{1}, x_{2}\right)\right)=\left[\begin{array}{cc}
g\left(x_{1}, x_{2}\right) & -m x_{1} \phi\left(k x_{1}\right) \phi^{\prime}\left(x_{2}\right) \\
0 & -\mu_{1}
\end{array}\right] \\
D f^{N}\left(p+\left(x_{1}, x_{2}\right)\right)=\left[\begin{array}{cc}
h\left(x_{1}, x_{2}\right) & (-1)^{r(N)} \lambda_{1}^{N-1} m x_{1} \phi\left(k x_{1}\right) \phi^{\prime}\left(x_{2}\right) \\
0 & \mu
\end{array}\right]
\end{gathered}
$$

onde

$$
g\left(x_{1}, x_{2}\right)=-\lambda_{1}-m \phi\left(x_{2}\right)\left(\phi\left(k x_{1}\right)+k x_{1} \phi^{\prime}\left(k x_{1}\right)\right)
$$

$$
h\left(x_{1}, x_{2}\right)=\lambda+(-1)^{r(N)} \lambda_{1}^{N-1} m \phi\left(x_{2}\right)\left(\phi\left(k x_{1}\right)+k x_{1} \phi^{\prime}\left(k x_{1}\right)\right)
$$

A partir deste ponto, dividimos a construção em dois casos.

Caso 1: Se $r(N)$ é par.

Neste caso $\lambda>1$ e $0<\mu<1$. Escolhemos $m$ como sendo $m=\frac{\lambda_{1}(1-2 \lambda)}{2 \lambda}<0$. Logo

$$
f^{N}\left(p+\left(x_{1}, x_{2}\right)\right)=p+\left(\lambda x_{1}+\left(\frac{1-2 \lambda}{2}\right) \phi\left(k x_{1}\right) \phi\left(x_{2}\right) x_{1}, \mu x_{2}\right)
$$


Vamos encontrar os pontos $N$-periódicos de $f$ em $U$. A equação

$$
f^{N}\left(p+\left(x_{1}, x_{2}\right)\right)=p+\left(x_{1}, x_{2}\right)
$$

é equivalente a

$$
\lambda x_{1}+\left(\frac{1-2 \lambda}{2}\right) \phi\left(k x_{1}\right) \phi\left(x_{2}\right) x_{1}=x_{1}, \text { e } \mu x_{2}=x_{2}
$$

Portanto um ponto $N$-periódico é $\left(x_{1}, x_{2}\right)=(0,0)$ (que corresponde a $p$ ) e os outros são $\left(x^{*}, 0\right)$ e $\left(-x^{*}, 0\right)$ (que correspondem aos pontos $p+\left(x^{*}, 0\right)$ e $p-\left(x^{*}, 0\right)$ ) onde $x^{*}$ é a solução positiva da equação

$$
\phi\left(k x_{1}\right)=\psi(\lambda) \operatorname{com} \psi(\lambda)=\frac{2(1-\lambda)}{1-2 \lambda} .
$$

Note que a equação anterior tem solução pois, para $\lambda>1,0<\psi(\lambda)<1$.

Para saber qual é a natureza destes pontos periódicos utilizamos a expressão 4.15 da derivada $D f^{N}$. Em particular

$$
D f^{N}(p+(0,0))=\left[\begin{array}{cc}
1 / 2 & 0 \\
0 & \mu
\end{array}\right]
$$

Logo $p$ é um ponto atrator para $f$. Também

$$
D f^{N}\left(p \pm\left(x^{*}, 0\right)\right)=\left[\begin{array}{cc}
h\left(x^{*}, 0\right) & \\
0 & \mu
\end{array}\right]=\left[\begin{array}{cc}
1+\left(\frac{1-2 \lambda}{2}\right) k x^{*} \phi^{\prime}\left(k x^{*}\right) & 0 \\
0 & \mu
\end{array}\right]
$$

Dado que $\lambda>1$ e $t \phi^{\prime}(k t)<0$ temos que os pontos $p \pm\left(x^{*}, 0\right)$ são pontos sela.

Proposição 4.1.2. O segmento aberto $\left\{p+(t, 0): t \in\left(-x^{*}, x^{*}\right)\right\}$ centrado em $p$ e o eixo vertical que passa por $p$ estão contidos na variedade estável bidimensional de $p, W^{s}\left(p, f^{N}\right)$.

Demonstração. Suponha que $0<x<x^{*}$ então $\phi(k x)>\phi\left(k x^{*}\right)$. Dado que $\lambda>1$ temos

$$
\lambda x+\left(\frac{1-2 \lambda}{2}\right) \phi(k x) x<x\left(\lambda+\left(\frac{1-2 \lambda}{2}\right) \phi\left(k x^{*}\right)\right)=x .
$$

Isto implica que $f^{n N}(p+(x, 0)) \rightarrow p$ quando $n \rightarrow \infty$. Para $-x^{*}<x<0$ a prova é a mesma.

Também $f^{N}(p+(0, t))=p+(0, \mu t)$ o qual implica que $f^{n N}(p+(0, t)) \rightarrow p$ quando $n \rightarrow \infty$.

Caso 2: Se $r(N)$ é impar.

Neste caso $\lambda<-1$ e $-1<\mu<0$. Defina $m=-\frac{\lambda_{1}(1+2 \lambda)}{2 \lambda}<0$. Logo

$$
f^{N}\left(p+\left(x_{1}, x_{2}\right)\right)=p+\left(\lambda x_{1}-\left(\frac{1+2 \lambda}{2}\right) \phi\left(k x_{1}\right) \phi\left(x_{2}\right) x_{1}, \mu x_{2}\right) .
$$


Fazendo uma análise similar ao caso anterior, provamos que existe um único ponto $N$-periódico atrator em $U$ que corresponde ao ponto $p$. A equação para os pontos $N$-periódicos em $U$ conduz às equações

$$
x_{1}\left(\lambda-\left(\frac{1+2 \lambda}{2}\right) \phi\left(k x_{1}\right) \phi\left(x_{2}\right)\right)=x_{1} \text { e } \mu x_{2}=x_{2} .
$$

Uma solução é $\left(x_{1}, x_{2}\right)=(0,0)$, que corresponde ao ponto $p$. Outras soluções devem ser $\left(x_{1}, 0\right)$ onde

$$
\phi\left(k x_{1}\right)=\psi(\lambda) \operatorname{com} \psi(\lambda)=\frac{2(\lambda-1)}{2 \lambda+1} .
$$

A anterior equação não tem soluções pois para $\lambda<-1$ temos $\psi(\lambda)>1$.

Pela escolha de $m$, a derivada de $f^{N}$ em $p$ é

$$
D f^{N}(p+(0,0))=\left[\begin{array}{cc}
-1 / 2 & 0 \\
0 & \mu
\end{array}\right]
$$

mostrando que $p$ é um ponto atrator.

Encontraremos os pontos de período $2 N$. Seja

$$
\sigma\left(x_{1}, x_{2}\right)=\lambda x_{1}-\left(\frac{1+2 \lambda}{2}\right) \phi\left(k x_{1}\right) \phi\left(x_{2}\right) x_{1} .
$$

Então $p+\left(x_{1}, x_{2}\right)$ é um ponto $2 N$-periódico se $x_{2}=0$ e

$$
\sigma\left(\sigma\left(x_{1}, 0\right), 0\right)=x_{1}
$$

Seja $\tau(t)=\sigma(t, 0)$. Então temos que encontrar as soluções de $\tau^{2}(t)=t$. Note que $\tau$ é uma função impar e, dado que $\phi(t) \leq 1$ e $t \phi^{\prime}(k t)<0$, temos

$$
\tau^{\prime}(t) \leq-\frac{1}{2}-\frac{1+2 \lambda}{2} t k \phi^{\prime}(t k)<0
$$

Logo $\tau^{2}$ é uma função crescente. Note que $t=0$ é a solução da equação que corresponde ao ponto $p$. Observe também que não existem soluções em $\left(0, \frac{\epsilon}{8 k}\right]$ e em $\left[\frac{\epsilon}{4 k}, \infty\right)$. Por tanto as soluções positivas estão em $\left[\frac{\epsilon}{8 k}, \frac{\epsilon}{4 k}\right]$. Dado que $\tau^{2}$ é crescente e $\tau\left(\frac{\epsilon}{4 k}\right)=\lambda^{2} \frac{\epsilon}{4 k}>\frac{\epsilon}{4 k}$ e $\tau^{2}\left(\frac{\epsilon}{8 k}\right)=\frac{\epsilon}{32 k}<\frac{\epsilon}{8 k}$, temos que, pelo teorema do valor intermediário, existe uma única solução $t^{*}$ em $\left[\frac{\epsilon}{8 k}, \frac{\epsilon}{4 k}\right]$. Como $\tau$ e impar, a outra solução é $-t^{*}$.

Portanto existem dois pontos $p \pm\left(t^{*}, 0\right)$ de período $2 N$. É facil ver que $f^{N}\left(p+\left(t^{*}, 0\right)\right)=p-\left(t^{*}, 0\right)$ e $f^{N}\left(p-\left(t^{*}, 0\right)\right)=p+\left(t^{*}, 0\right)$. A derivada nestes pontos é

$$
D f^{2 N}\left(p \pm\left(t^{*}, 0\right)\right)=\left[\begin{array}{cc}
\left(-1-k \frac{1+2 \lambda}{2} t^{*} \phi^{\prime}\left(k t^{*}\right)\right)^{2} & 0 \\
0 & \mu^{2}
\end{array}\right]
$$

Isto mostra que estes pontos são tipo sela pois $k \frac{1+2 \lambda}{2} t^{*} \phi^{\prime}\left(k t^{*}\right)>0$. 
Proposição 4.1.3. O segmento aberto $p+\left\{(t, 0), t \in\left(-t^{*}, t^{*}\right)\right\}$ e o eixo vertical que passa por $p$ estão contidos na variedade estável bidimensional $W^{s}\left(p, f^{N}\right)$.

Demonstração. A prova é similar à da proposição 4.1.2.

\subsubsection{Hiperbolicidade de $\Omega(f)$ para $f$.}

Definamos o conjunto $\Lambda_{f}:=\left(R_{0} \cup R_{1}\right) \backslash W^{s}(p)$. Se não há lugar a confusão, simplesmente escreveremos $\Lambda$ em vez de $\Lambda_{f}$. É claro que $\Omega(f) \backslash\{p\} \subset \Lambda$. Observe que $\Lambda$ é um conjunto compacto contido na união de $R_{0}$ e $R_{1}$.

Nesta subseção provaremos que, escolhendo $k$ convenientemente, em $\Lambda$ existe um campo de cones para $f^{N}$ ou para $f^{2 N}$ (dependendo de $r(N)$ ) e, portanto, pelo teorema abaixo de Newhouse [New04], $\Omega(f)$ é hiperbólico para $f$.

Teorema 4.1.1 (Newhouse). Uma condição necessária e suficiente para que $\Lambda$ seja uniformemente hiperbólico para $f$ é que exista um enteiro $N>0$ e um campo de cones invariante $\mathcal{C}$ tal que $f^{N}$ expande e co-expande vetores em $\mathcal{C}$.

Sejam $q_{1}=p-\left(x^{*}, 0\right)$ e $q_{1}=p+\left(x^{*}, 0\right)$ se $N$ é par, ou $q_{1}=p-\left(t^{*}, 0\right)$ e $q_{2}=p+\left(t^{*}, 0\right)$ se $N$ é impar. Das proposições 4.1 .2 e 4.1.3 temos

Proposição 4.1.4. As variedades unidimensionais $W^{u}\left(q_{1}, f\right)$ e $W^{u}\left(q_{2}, f\right)$ limitam a bacia de atração de p.

Provaremos a seguinte proposição

Proposição 4.1.5. Para $k$ suficientemente grande $\Lambda$ é um conjunto hiperbólico para $f$.

Se um ponto $q \in \Lambda \backslash U$ então

$$
\text { ou } \left.D f(q))=\left[\begin{array}{cc}
-\lambda_{1} & 0 \\
0 & -\mu_{1}
\end{array}\right] \text { ou } D f(q)\right)=\left[\begin{array}{cc}
\lambda_{1} & 0 \\
0 & \mu_{1}
\end{array}\right]
$$

Precisaremos a expressão da derivada de $f^{N}$ em um ponto $q=(x, y)$ de $\Lambda$. Pela definição de $f$ deduzimos que

$$
D f^{N}(q)=\left[\begin{array}{cc}
A(x, y) & B(x, y) \\
0 & \pm \mu
\end{array}\right]
$$

Para os outros termos observamos o seguinte:

a) Se $f^{i}(q) \notin U$ para $i=0, N-1$ então $A(x, y)= \pm \lambda$ e $B(x, y)=0$.

b) Se $q \in U$ então

$$
A(x, y)=h\left(x_{1}, y_{1}\right) \text { e } B(x, y)=(-1)^{r(N)} \lambda_{1}^{N-1} m x_{1} \phi\left(k x_{1}\right) \phi^{\prime}\left(x_{2}\right) .
$$

c) Se existe $i \in\{1, . ., N-1\}$ tal que $f^{i}(q) \in U$ então

$$
A(x, y)=\lambda+(-1)^{r(N)} \lambda_{1}^{N-1} m \phi(v)(\phi(k u)+k u \phi(k u))
$$


e

$$
B(x, y)=(-1)^{r(N)} \lambda_{1}^{N-1-i} \mu_{1}^{i} m u \phi(k u) \phi^{\prime}(u)
$$

onde $(u, v)$ são as coordenadas de $f^{i}(q)$ em $U$.

No que segue suponha que $r(N)$ é par.

Lema 4.1.1. Existe $\lambda^{\prime}>1$ tal que $|A(x, y)|>\lambda^{\prime}$ em $\Lambda$.

Demonstração. Se $q$ é como no caso a) a afirmação do lema é verdade. Suponha que $q$ é como no caso b). Note que neste caso é suficiente mostrar que $h\left(x_{1}, x_{2}\right)>$ 1 no complemento de um conjunto compacto de $R_{0} \cup R_{1} \backslash \Lambda=\left(R_{0} \cup R_{1}\right) \cap$ $W^{s}(p, f)$. Seja $\left.V:=\left\{\left(x_{1}, x_{2}\right)\right\} \in U: h\left(x_{1}, x_{2}\right) \leq 1\right\}=\bigcup_{\left(x_{1}, x_{2}\right) \in U}\left(V_{x_{2}} \times\left\{x_{1}\right\}\right)$ onde $V_{x_{2}}=\left\{x_{1}: h\left(x_{1}, x_{2}\right) \leq 1\right\}$. Note que $h\left(x_{1}, x_{2}\right) \leq 1$ se e somente se

$$
\frac{d[t \phi(k t)]}{d t} \mid t=x_{1} \geq \frac{2(1-\lambda)}{1-2 \lambda} \cdot \frac{1}{\phi\left(x_{2}\right)} .
$$

Assim, dado que $\frac{d[t \phi(k t)]}{d t}$ é par temos que $V_{x_{2}}$ é um intervalo simétrico para todo $x_{2}$ e, se $0 \leq y<x$ então $\phi(x) \leq \phi(y)$ e, por tanto, $V_{x} \subset V_{y}$. Lembre que

$$
f^{N}\left(p+\left(x_{1}, x_{2}\right)\right)=p+\left(x_{1}\left(h\left(x_{1}, x_{2}\right)-\left(\frac{1-2 \lambda}{2}\right) \phi\left(x_{2}\right) \phi^{\prime}\left(k x_{1}\right) k x_{1}\right), \mu x_{2}\right)
$$

e que $f^{N}$ preserva os níveis horizontais. Dado que

$$
\lambda+\left(\frac{1}{2}-\lambda\right) \phi\left(x_{2}\right) \phi\left(k x_{1}\right) \geq \frac{1}{2}
$$

temos que

$$
\frac{1}{2} \leq h\left(x_{1}, x_{2}\right)-\left(\frac{1-2 \lambda}{2}\right) \phi\left(x_{1}\right) \phi^{\prime}\left(k x_{1}\right) k x_{1} \leq 1 .
$$

Então, pela equação anterior, se $f^{N}\left(x_{1}, x_{2}\right)=\left(x_{1}^{\prime}, x_{2}^{\prime}\right)$ e $f^{N}\left(V_{x_{2}} \times\left\{x_{2}\right\}\right)=$ $V_{x_{2}^{\prime}}^{\prime} \times\left\{x_{2}^{\prime}\right\}$ teremos que $V_{x_{2}^{\prime}}^{\prime} \subset V_{x_{2}^{\prime}}$. Portanto, temos provado que $f^{N}(V) \subset V$.

Por outro lado, note que $V_{0}$ contem $x_{1}=0$, pois $h(0,0)=1 / 2$, e não contem $x_{1}=x^{*}$ pois $h\left(x^{*}, 0\right)=1+\left(\frac{1-2 \lambda}{2}\right) k x^{*} \phi\left(k x^{*}\right)>1$. Logo existe uma vizinhança $I$ de 0 tal que $V_{t} \subset W^{s}(p, f)$ para todo $t \in I$.

Assim, existe um $n$ tal que $f^{n N}(V) \subset W^{s}(f, p)$ e portanto $V \subset W^{s}(f, p)$.

$\mathrm{O}$ caso c) segue utilizando um procedimento similar. A única diferença é que $V$ está no ponto $f^{-i}(p)$.

Prova da proposição 4.1.5. . Desde que $\phi^{\prime}(t)$ é limitada e $\phi<1$ temos que existe uma constante $C_{1}$ tal que $|B(x, y)|<\frac{C_{1} \epsilon}{4 k}$. Dado que $A(x, y)$ é uma função limitada em $\Lambda$, existe $M$ tal que $|A|<M$. Seja $\lambda^{\prime}$ como no lemma 4.1.1. Tome $0<\gamma<1$ e $\delta>0$ tão pequenos tal que $\lambda^{\prime}-\mu>\delta$ e $\lambda^{\prime 2}-\gamma^{2}>1+2 \gamma \delta M$ e escolha $k$ tão grande que $|B(x, y)|<\delta$.

Considere os cones horizontais da forma $C^{u}(q)=\{(u, v):|v|<\gamma|u|\}$. Estes 
cones são invariantes para $f^{N}$ pois se $D f_{q}^{N}(u, v)=:\left(u^{\prime}, v^{\prime}\right)$ então:

$$
\left|u^{\prime}\right|=|A u+B v| \geq|A||u|-|B||v|>\frac{\lambda^{\prime}}{\gamma}|v|-\delta|v|=\frac{\lambda^{\prime}-\delta \gamma}{\gamma \mu}\left|v^{\prime}\right|
$$

Como $\lambda^{\prime}-\delta \gamma>\mu$ então $\left|u^{\prime}\right|>\frac{1}{\gamma}\left|v^{\prime}\right|$. Para ver que vetores nos $\gamma$-cones são expandidos notamos que

$$
\begin{aligned}
\left|u^{\prime}\right|^{2}+\left|v^{\prime}\right|^{2} & =(A u+B v)^{2}+\mu^{2} v^{2} \\
& \geq|A|^{2}|u|^{2}-2|A||B||u \| v|+|B|^{2}|v|^{2}+\mu^{2}|v|^{2} \\
& \geq \lambda^{\prime 2}|u|^{2}-2 \gamma|A||B||u|^{2}-\gamma^{2}|u|^{2}+|v|^{2} \\
& \geq\left(\lambda^{\prime 2}-2 \gamma \delta|A|-\gamma^{2}\right)|u|^{2}+|v|^{2} \\
& \geq\left(\lambda^{\prime 2}-2 \gamma \delta M-\gamma^{2}\right)|u|^{2}+|v|^{2} \\
& \geq \beta|u|^{2}+|v|^{2}>|u|^{2}+|v|^{2}
\end{aligned}
$$

onde $\beta>1$ pela escolha dos parâmetros.

Similarmente podemos mostrar que cones verticais são invariantes por $D\left(f^{N}\right)^{-1}$ e que $D\left(f^{N}\right)^{-1}$ expande vetores nestes cones verticais. Portanto, a hiperbolicidade de $f$ segue pelo teorema 4.1.1.

Se $r(N)$ é impar a prova da hiperbolicidade de $\Lambda$ para $f^{N}$ segue as mesmas ideias.

\subsubsection{Relação entre $\Omega(f)$ e $\Sigma_{2}$.}

Note que $R_{0}$ e $R_{1}$ é uma partição de Markov para $f$. Assim, dada uma sequência simbólica $s=\left(s_{i}\right)_{i \in \mathbb{Z}} \in \Sigma_{2}$, o conjunto $\mathcal{O}_{s}:=\cap_{i=-\infty}^{\infty} f^{-i}\left(R_{s_{i}}\right)$ é um conjunto compacto não vazío. Defina $\mathcal{O}=\bigcup_{s \in \Sigma_{2}} O_{s}$ então $\mathcal{O}$ contém a $\Omega(f)$, pois cada ponto fora de $R_{0} \cup R_{1}$ é errante para $f$ e portanto cada ponto de $\Omega(f)$ segue um itinerário em $\Sigma_{2}$. Assim, o seguinte diagrama comuta

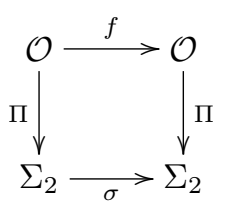

onde $\Pi$ é o itinerário de um ponto de $\mathcal{O}$ nos retângulos $R_{0}$ e $R_{1}$. Note que $\Pi^{-1}(\{s\})=\mathcal{O}_{s}$ e $f\left(\mathcal{O}_{s}\right)=\mathcal{O}_{\sigma(s)}$.

O problema é determinar quando $O_{s}$ contém um único ponto de $\Omega(f)$. Em geral isto não é o caso: se $r(N)$ é par e $s=c_{P}^{\infty}$ então $\mathcal{O}_{s}$ contém o segmento $\left\{p+(t, 0): t \in\left[-x^{*}, x^{*}\right]\right\}$. Mas, provaremos que, para a maioria das sequências, $\mathcal{O}_{s}$ é unitario e o único ponto que contém pertence a $\Omega(f)$.

Dada uma sequência $s \in \Sigma_{2}$ e $m \in \mathbb{N}$, definamos a $m$-faixa estável de $s$, 
$\mathcal{F}_{m}^{s}(s)$, pelo conjunto

$$
\mathcal{F}_{m}^{s}(s)=\bigcap_{i=0}^{m} f^{-i}\left(R_{s_{i}}\right)
$$

e a $m$-faixa instável de s pelo conjunto

$$
\mathcal{F}_{m}^{u}(s)=\bigcap_{i=-m}^{0} f^{-i}\left(R_{s_{i}}\right) .
$$

É claro que $\mathcal{O}_{s}=\mathcal{F}_{\infty}^{s}(s) \cap \mathcal{F}_{\infty}^{u}(s)$.

Proposição 4.1.6. Para cada $s \in \Sigma_{2}$ e $m \in \mathbb{N}$, temos que:

a) $\mathcal{F}_{m}^{s}(s)$ é uma faixa vertical que cruza $R_{s_{0}}$,

b) a interseção de $\mathcal{F}_{m}^{s}(s)$ e toda linha horizontal em $R_{s_{0}}$ é um intervalo horizontal contido em $R_{s_{0}}$,

c) $\mathcal{F}_{m}^{u}(s)$ é uma faixa horizontal de lados paralelos ao eixo $x$ que cruza $R_{s_{0}}$,

d) $\mathcal{F}_{\infty}^{u}(s)$ é um intervalo horizontal que cruza $R_{s_{0}}$,

e) $\mathcal{O}_{s}=[a, b] \times\left\{y_{s}\right\}$ é um segmento horizontal em $R_{s_{0}}$, onde $y_{s}$ depende unicamente de ...s $s_{-2} s_{-1}$.

Demonstração. Provaremos a) e b) juntos por indução. Se $m=1$ então a afirmação é verdade pois $f^{-1}=F^{-1}$ em $\partial R_{i}$, para $i=0,1$, e $\partial_{s} R_{i} \cap f(U)=\emptyset$. Suponha que $\mathcal{F}_{m-1}^{s}(s)$ satisfaz a) e b), então $\mathcal{F}_{m}^{s}(s)=\mathcal{F}_{m-1}^{s}(s) \cap f^{-m}\left(R_{s_{m}}\right)$ satisfaz a) pois $f=F$ em $\partial_{u} R_{i}$ e $f^{-m}\left(\partial_{u} R_{i}\right)=F^{-m}\left(\partial_{u} R_{i}\right)$, e satisfaz $\left.\mathbf{b}\right)$ pois a segunda coordenada de $f$ é igual a segunda coordenada de $F$.

A prova de c) e d) segue utilizando uma prova similar à anterior e lembrando que $f$ contrai por um fator $\mu_{1}$ a segunda coordenada.

O item e) segue por $\mathbf{b}$ ) e $\mathbf{d}$ ).

Queremos demonstrar a seguinte proposição:

Proposição 4.1.7. Seja $s \in \Sigma_{2}$.

a) Se $s=\ldots . s_{0} \ldots s_{n} c_{P}^{\infty}$ então $\mathcal{O}_{s}=[r, t] \times\left\{y_{s}\right\} e(r, t) \times\left\{y_{s}\right\} \subset W^{s}(p, f)$ onde $\left(r, y_{s}\right) \in W^{s}\left(p+\left(-x^{*}, 0\right)\right)$ e $\left(t, y_{s}\right) \in W^{s}\left(p+\left(x^{*}, 0\right)\right)$ ou viceversa.

b) $S e s \neq \ldots s_{0} \ldots s_{n} c_{P}^{\infty}$ então $\mathcal{O}_{s}$ é unitário.

Para a sua prova precisamos do lema

Lema 4.1.2. Seja $p=\left(p_{x}, p_{y}\right)$. Se $r(N)$ é par então $\mathcal{O}_{c_{P}^{\infty}}=\{p+(t, 0): t \in$ $\left.\left[-x^{*}, x^{*}\right]\right\} \times\left\{p_{y}\right\}$. Se $r(N)$ é impar então $\mathcal{O}_{c_{P}^{\infty}}=\left\{p+(t, 0): t \in\left[-t^{*}, t^{*}\right]\right\} \times\left\{p_{y}\right\}$. 
Demonstração. Provaremos para $r(N)$ par. O caso $r(N)$ impar é similar e a prova será omitida.

Seja $I=\left\{p+(t, 0): t \in\left[-x^{*}, x^{*}\right]\right\} \times\left\{p_{y}\right\}$ e $s=c_{P}^{\infty}$. Dado que $I \subset \mathcal{O}_{s}$ e $\mathcal{O}_{s}$ é um intervalo, podemos supor que $\mathcal{O}_{s}=\{p+(t, 0): t \in[a, b]\} \times\left\{p_{y}\right\}$ onde $a \leq-x^{*}$ e $x^{*} \leq b$. Suponha que $b>x^{*}$. Tome $q$, $\tilde{q}$ tais que $p_{x}+x^{*}<q_{x}<\tilde{q}_{x}<$ $p_{x}+b$.

Se $f^{k N}\left([q, \tilde{q}] \times\left\{p_{y}\right\}\right) \cap W^{s}(p, f)=\emptyset, \forall k \geq 0$ então $f^{k N}\left([q, \tilde{q}] \times\left\{p_{y}\right\}\right) \subset \Lambda ;$ mas $\Lambda$ é hiperbólico e por tanto (pelo lema 4.1.1)

$$
\left|f^{k N}(q)-f^{k N}(\tilde{q})\right| \geq\left(\min \left|D f^{N}\right|^{k}\right)|q-\tilde{q}| \geq\left(\lambda^{\prime}\right)^{k}\left|q_{x}-\tilde{q}_{x}\right|
$$

Seja $\left|R_{i}\right|_{x}$ a longitude do lado instável de $R_{i}$. Tomando $k_{0}$ tal que

$$
\left|f^{k_{0} N}(q)-f^{k_{0} N}(\tilde{q})\right|>\max \left\{\left|R_{0}\right|_{x},\left|R_{1}\right|_{x}\right\}
$$

chegamos a uma contradição pois neste caso ou $q$ e $\tilde{q}$ estão en retângulos diferentes ou um deles está fora de $R_{0} \cup R_{1}$. Logo, existe $k_{1}$ tal que $f^{k_{1} N}([q, \tilde{q}] \times$ $\left.\left\{p_{y}\right\}\right) \cap W^{s}(p, f) \neq \emptyset$.

Assim, existe um segmento $\mathcal{W} \subset\left[x^{*}, b\right] \times\left\{p_{y}\right\}$ tal que $f^{k_{1} N}(\mathcal{W}) \subset W^{s}(p)$. Dado que $\mathcal{W} \subset \mathcal{O}_{s}$ e $f$ preserva os níveis horizontais, existe um $k_{2}$ tal que $f^{k_{2} k_{1} N}(\mathcal{W}) \subset I$. Mas $f^{N}(I)=I=f^{-N}(I), \log \mathcal{W} \subset I$. Isto é uma contradição. Por tanto $b=x^{*}$.

Da mesma maneira provamos que $a=-x^{*}$.

Prova da proposição 4.1.7. Sem perda de generalidade podemos supor que $s=$ $\ldots s_{-1} \cdot c_{P}^{\infty}$ então $\mathcal{O}_{s}=\mathcal{F}_{\infty}^{s}\left(c_{P}^{\infty}\right) \cap \mathcal{F}_{\infty}^{u}(s)$. Pela lema anterior, $\mathcal{F}_{\infty}^{s}\left(c_{P}^{\infty}\right)$ é uma faixa vertical em $R_{1}$ cuja interseção com a reta horizontal que passa por $p$ é $\mathcal{O}_{c_{P}}=I$. Note que $f^{k N}\left(\mathcal{F}_{\infty}^{s}\left(c_{P}^{\infty}\right)\right)=\mathcal{F}_{k N}^{u}\left(c_{P}^{\infty}\right) \cap \mathcal{F}_{\infty}^{s}\left(c_{P}^{\infty}\right)$. Então

$$
\lim _{k \rightarrow \infty} f^{k N}\left(\mathcal{F}_{\infty}^{s}\left(c_{P}^{\infty}\right)\right)=\mathcal{O}_{c_{P}}
$$

Tomando $k$ suficientemente grande tal que $\mathcal{G}:=f^{k N}\left(\mathcal{F}_{\infty}^{s}\left(c_{P}^{\infty}\right)\right)$ está próximo de $\mathcal{O}_{c_{P}}$ e pela natureza sela dos pontos $p \pm\left(x^{*}, 0\right)$ temos que $\partial_{s} \mathcal{G}$ (bordo vertical) deve estar contido nas variedades estáveis de $p \pm\left(x^{*}, 0\right)$. Logo o bordo estável de $\mathcal{F}_{\infty}^{s}\left(c_{P}^{\infty}\right)$ (bordo vertical) está formado pelas variedades estáveis de $p \pm\left(x^{*}, 0\right)$. Então as sequências $s=\ldots s_{-1} \cdot c_{P}^{\infty}$ satisfazem a). Provaremos que são as únicas que satisfazem a).

Seja $s$ tal que $\mathcal{O}_{s} \cap W^{s}(p) \neq \emptyset$ então existe um ponto $q \in \mathcal{O}_{s}$ tal que $\Pi(q)=\ldots a_{n} c_{P}^{\infty}$. Mas $\Pi(q)=s$ então $s=\ldots a_{n} c_{P}^{\infty}$ e, pelo feito antes, $\mathcal{O}_{s}$ satisfaz a).

Se $s$ é tal que $\mathcal{O}_{s} \cap W^{s}(p)=\emptyset$ então $f^{j}\left(\mathcal{O}_{s}\right) \subset \Lambda$ e portanto a distancia entre dois pontos de $\mathcal{O}_{s}$ cresce pelo menos por $\lambda^{\prime}$ por iterados de $f^{N}$ (lema 4.1.1). Isto implica que $\mathcal{O}_{s}$ é unitário e que $s \neq \ldots . . . s_{0} \ldots s_{n} c_{P}^{\infty}$, com o qual provamos b).

Seja $\Omega^{\prime}=\Omega(f) \backslash\{p\}$, então $\Omega^{\prime} \subset \Lambda$ e portanto é hiperbólico. Das definições temos que $\Omega^{\prime} \subset \bigcup_{s \in \Sigma_{2}} \partial \mathcal{O}_{s}$. Desejamos demonstrar que $\Omega^{\prime}=\bigcup_{s \in \Sigma_{2}} \partial \mathcal{O}_{s}$. 
Lema 4.1.3. $\bigcup_{s \in \Sigma_{2}} \partial \mathcal{O}_{s} \subset \Omega^{\prime}$.

Demonstração. Se $s \neq \ldots s_{0} \ldots s_{n} c_{P}^{\infty}$ então pela proposição $4.1 .7, \partial \mathcal{O}_{s}=\mathcal{O}_{s}=$ $\left\{o_{s}\right\}$. Dado $\epsilon>0$ pequeno, escolha $m$ tal que $\left|\mathcal{F}_{m}^{s}(s) \cap \mathcal{F}_{\infty}^{u}(s)\right|<\epsilon / 2$. Logo, escolha $m^{\prime}$ tal que $\operatorname{diam}\left(\mathcal{F}_{m}^{s}(s) \cap \mathcal{F}_{m^{\prime}}^{u}(s)\right)<\epsilon$. Note que a sequência periódica $t$ correspondente a repetição bi-infinita de $s_{-m^{\prime}} \ldots s_{-1} \cdot s_{0} \ldots s_{m}$ é diferente das sequências do tipo $\ldots a_{n} c_{P}^{\infty}$. Assim, $\mathcal{O}_{t}=\left\{o_{t}\right\}$ onde $o_{t}$ é um ponto $\left(m+m^{\prime}+1\right)$ periódico de $f$ e $\operatorname{dist}\left(o_{s}, o_{t}\right)<\epsilon$. Portanto, $o_{s} \in \Omega^{\prime}$ pois em toda vizinhança de $o_{s}$ existem pontos periódicos.

Se $s=c_{P}^{\infty}$ então $\partial \mathcal{O}_{s}=\left\{p-\left(x^{*}, 0\right), p+\left(x^{*}, 0\right)\right\} \subset \Omega^{\prime}$.

Se $s=\ldots s_{0} \ldots s_{n} c_{P}^{\infty} \neq c_{P}^{\infty}$ então $\partial \mathcal{O}_{s}=\left\{\left(r, y_{s}\right),\left(t, y_{s}\right)\right\}$ onde $\left(r, y_{s}\right) \in W^{s}(p-$ $\left.\left(x^{*}, 0\right)\right)$ e $\left(t, y_{s}\right) \in W^{s}\left(p+\left(x^{*}, 0\right)\right)$ ou viceversa. Sem perda de generalidade suponha que $s=\ldots \cdot c_{P}^{\infty}$. Escolha $m$ tal que se $\left\{\left(r^{\prime}, y_{s}\right),\left(t^{\prime}, y_{s}\right)\right\}:=\partial\left(\mathcal{F}_{\infty}^{u}(s) \cap\right.$ $\left.\mathcal{F}_{m}^{s}(s)\right)$ então $\left|r^{\prime}-r\right|<\epsilon / 2$ e $\left|t-t^{\prime}\right|<\epsilon / 2$. Depois escolha $m^{\prime}$ tal que

$$
\operatorname{dist}\left(\partial\left(\mathcal{F}_{m^{\prime}}^{u}(s) \cap \mathcal{F}_{m}^{s}(s)\right), \partial\left(\mathcal{F}_{m^{\prime}}^{u}(s) \cap \mathcal{F}_{\infty}^{s}(s)\right)\right)<\epsilon .
$$

Seja $s^{\prime}$ a sequéncia periódica correspondente a repetição bi-infinita de $s_{-m^{\prime}} \ldots$. $s_{0} \ldots s_{m}$. A sequência $s^{\prime}$ é diferente das sequências periódicas $\ldots . . . a_{n} c_{P}^{\infty}$ então $\mathcal{O}_{s^{\prime}}=\left\{o_{s^{\prime}}\right\}$. Logo $o_{s^{\prime}}$ está proximo ou de $\left(r, y_{s}\right)$ ou de $\left(t, y_{s}\right)$. Escolhendo $m$ e $m^{\prime}$ convenientemente podemos fazer que $o_{s^{\prime}}$ esteja próximo de $\left(r, y_{s}\right)$ ou de $\left(t, y_{s}\right)$ e assim, $\left(r, y_{s}\right)$ e $\left(t, y_{s}\right)$ pertencem a $\Omega^{\prime}$ pois podem ser aproximados por órbitas periódicas.

Assim, para o derivado da ferradura $f$, cada sequência em $\Sigma_{2}$ corresponde a uma única órbita em $\Omega(f)=\Omega^{\prime} \cup\{p\}$ se a sequência não esta na variedade estável da sequência $c_{P}^{\infty}$. Em outro caso, cada sequência corresponde a dois (ou três, se a sequência é $c_{P}^{\infty}$ ) pontos de $\Omega(f)=\Omega^{\prime} \cup\{p\}$. Disto deduzimos que a configuração das variedades invariantes de $f$ é dada como na ferradura com a variedade estável de $p$ bifurcando em duas variedades estáveis: as correspondentes aos pontos selas $p \pm\left(x^{*}, 0\right)$ (no caso $r(N)$ par). Como na ferradura, as variedades invariantes de cada ponto são densas em $\Omega(F)$ e portanto a bacia de atração de um elemento da órbita $P$ é densa em $\Omega(f)$.

\subsection{O teorema de poda para o derivado da ferradura $f$}

Uma vez construido o DF, podemos fazer podas do derivado da ferradura utilizando as mesmas ideias do capítulo 3, isto é, encontrando regiões livres; mas também, neste caso, existem outros tipos de regiões com as quais podemos construir difeos de poda. Estas serão chamadas regiões atratoras. Note que as condições de poda para o DF estão relaciondas as condições gerais de poda dadas em [dC99] (Veja a seção 2.3.2). Sem embargo, para discos de poda de DF's, a isotopia de poda é uma difeotopia obtida fazendo só uma difeotopia (ou um número finito de isotopias em caso de coleções de discos de poda).

A seguinte definição será importante.

Definição 4.2.1. Sejam $A$ e $B$ duas regiões simplesmente conexas tais que $A \subset B$. Dizemos que $A$ é livre para $f$ em $B$ se $f^{k}(A) \cap B=\emptyset, \forall k \geq 1$. Dizemos 
que $A$ é atratora para $f$ em $B$ se existe um $j \in \mathbb{N}$ tal que $f^{i}(A) \cap B=\emptyset$, $\forall i=1, . ., j-1$ e $f^{j}(A) \subset A$. Podemos fazer as mesmas definições para $f^{-1}$ substituindo $f$ por $f^{-1}$.

Seja $f$ o difeomorfismo DA, como na seção 4.1, construido utilizando a órbita periódica de código $c_{P}$. A partir de $f$ podemos definir dois tipos de discos de poda.

\subsubsection{Tipo periódico-homoclínico}

Seja $D$ um disco limitado por um segmento de variedade estável do ponto $p \pm\left(x^{*}, 0\right)$ e um segmento de variedade instável do ponto $0^{\infty}$; ou seja, $D$ é como um disco homoclínico do capítulo 3 , limitado por variedades invariantes e simétrico em relação a uma horizontal traçada no meio do quadrado que define a ferradura, mas com o lado estável pertencendo a variedade estável de $p \pm\left(x^{*}, 0\right)$. Daremos condições para que um destes discos seja de poda. Como na definição de disco homoclínico, podemos definir a região instável $L$ de $D$ limitada por segmentos de variedade estável (um deles é o bordo estável de $D$ ) e que não contém pontos do conjunto não errante. Definimos também a região $U$ limitada pelo bordo instável de $D$ e outro segmento de variedade instável ao $0^{\infty}$ tal que não existem pontos do conjunto não-errante em $U$ (Veja as figuras 4.2(a) e 4.2(b)). Assim $L$ e $U$ satisfazem

$$
\lim _{k \rightarrow \infty} f^{k}(L)=p \text { e } \lim _{n \rightarrow \infty} f^{-k}(U)=\infty
$$

Temos dois casos.

\section{Caso 1:}

No primeiro caso $D$ é um disco de poda se $L$ é livre para $f$ em $D$ e $U$ é livre para $f^{-1} \mathrm{em} D$. A figura 4.2(a) mostra uma representação de um destes discos. A região $L$ é a região $A G E F H B C D A$ e a região $U$ é $A G H B C F E D A$. $\mathrm{Na}$ figura 4.3 mostramos um exemplo destes discos quando o ponto periódico no qual construimos o derivado da ferradura tem codificação simbólica $(101)^{\infty}$.

\section{Caso 2:}

Neste caso $D$ é um disco de poda se $L$ é uma região atratora para $f$ em $D$ e se $U$ é livre para $f^{-1}$ em $D$. Neste caso, o ponto $p$ está em $L$. Observe que $j=N$ ou $j=2 N$ onde $N$ é o período da sequência $c_{P}$. Na figura 4.2(b) mostramos um destes discos. O ponto $p$ está representado por um quadrado azul. A região $L$ é a região $A G E F H B C D A$ e a região $U$ é $A G H B C F E D A$. Na figura 4.4 mostramos um exemplo destes discos quando o ponto periódico utilizado para construir o DF é $(101)^{\infty}$. 


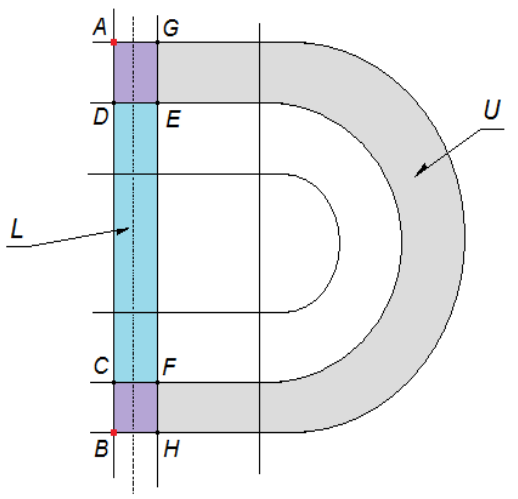

(a) Caso 1 .

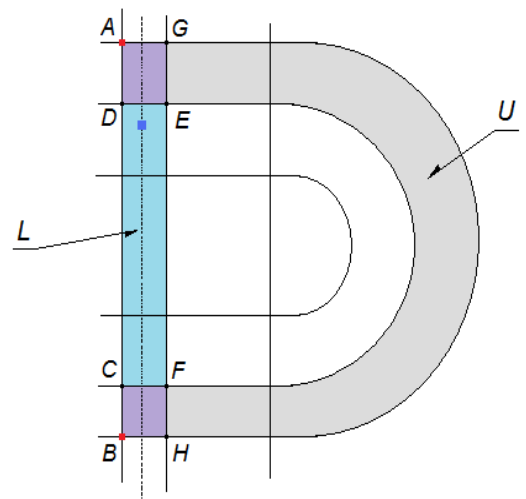

(b) Caso 2.

Figura 4.2: Discos do tipo periódico homoclínico. A figura 4.2(a) corresponde a uma região livre e a figura 4.2 (b) é quando $L$ é atratora.

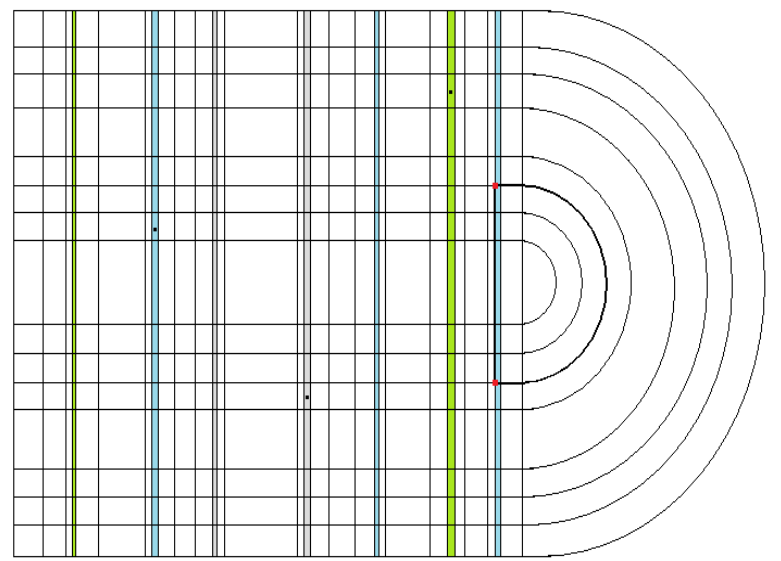

Figura 4.3: Exemplo

\subsubsection{Tipo periódico-periódico}

Sem perda de generalidade podemos supor que as variedades estáveis de pontos hiperbólicos de $f$ são verticais e que as variedades instáveis são horizontais.

Escolhendo outra órbita periódica $Q$ de período $N_{1}$, podemos definir novamente um derivado de $f$ modificando a variedade instável de $q$, onde $q$ é o ponto de maior altura da órbita $Q$. Isto é, modificando a coordenada local $y$ em $q$.

O difeomorfismo obtido depois desta nova deformação, chamado novamente de $f$, tem treis novos pontos numa vizinhança de $q$ : um ponto repulsor $q$ e dois pontos hiperbólicos $q \pm\left(0, y^{*}\right)$. Fazendo uma análise similar a subseção 4.1.4 


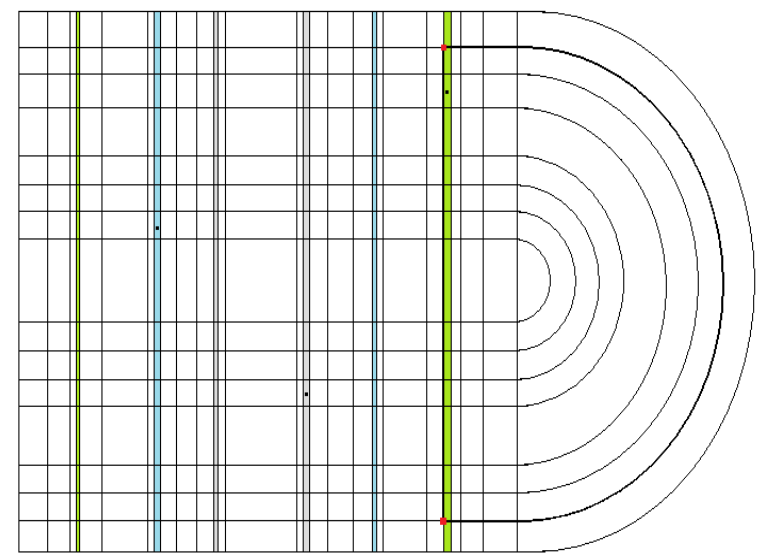

Figura 4.4: Exemplo.

podemos mostrar que existe uma semiconjugação, finita a um, entre $\Omega(f)$ e $\Sigma_{2}$.

Os discos homoclínicos para este $f$, então, são limitados por um segmento de variedade estável do ponto $p \pm\left(x^{*}, 0\right)$ e um segmento da variedade instável de $q \pm\left(0, y^{*}\right)$.

Desta vez, as regiões $L$ e $U$ são definidas utilizando segmentos da variedade estável de $p \pm\left(x^{*}, 0\right)$ e segmentos da variedade instável de $q \pm\left(0, y^{*}\right)$, respectivamente; e tais que não existem pontos do conjunto não errante de $f$ em $L \mathrm{e}$ em $U$. Então elas satisfazem

$$
\lim _{k \rightarrow \infty} f^{k}(L)=p \text { e } \lim _{k \rightarrow \infty} f^{-k}(U)=q .
$$

As condições para que um disco homoclínico seja de poda estão discriminadas nos seguintes casos. Em todos eles a região $L$ é a região $A G E F H B C D A$ e a região $U$ é $A G H B C F E D A$.

\section{Caso 1:}

$D$ é um disco de poda se $L$ é livre para $f$ em $D$ e $U$ é livre para $f^{-1}$ em $D$. Veja figura 4.5(a).

\section{Caso 2:}

$D$ é um disco de poda se $L$ é atratora para $f$ em $D$ e $U$ é livre para $f^{-1}$ em $D$. Veja figura $4.5(\mathrm{~b})$.

\section{Caso 3:}

$D$ é um disco de poda se $L$ é livre para $f$ em $D$ e $U$ é atratora para $f^{-1}$ em $D$. Veja figura 4.6(a). 


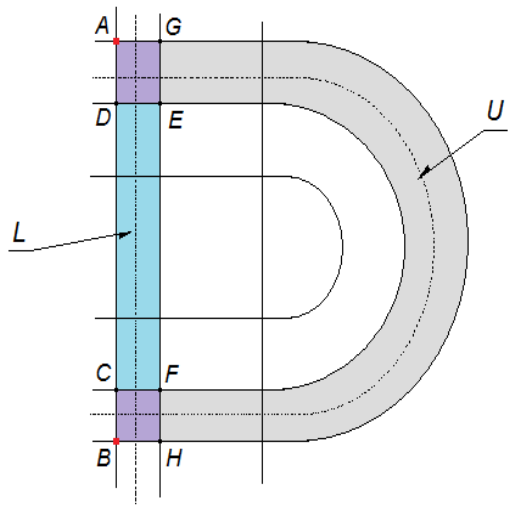

(a) Caso 1 .

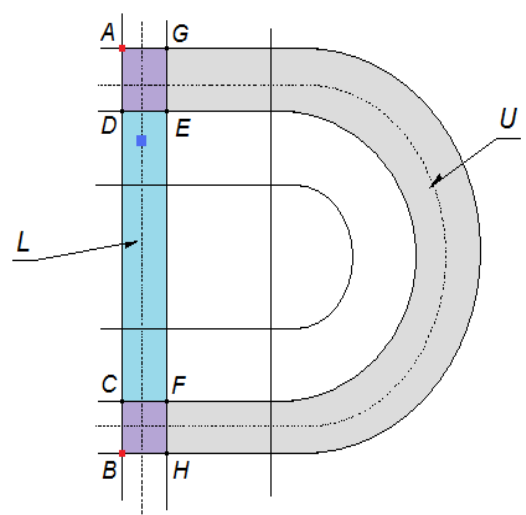

(b) Caso 2.

Figura 4.5: Discos do tipo periódico-periódico.

\section{Caso 4:}

$D$ é um disco de poda se tanto $L$ como $U$ são atratoras em $D$ para $f$ e $f^{-1}$, respectivamente. Veja figura $4.6(\mathrm{~b})$.

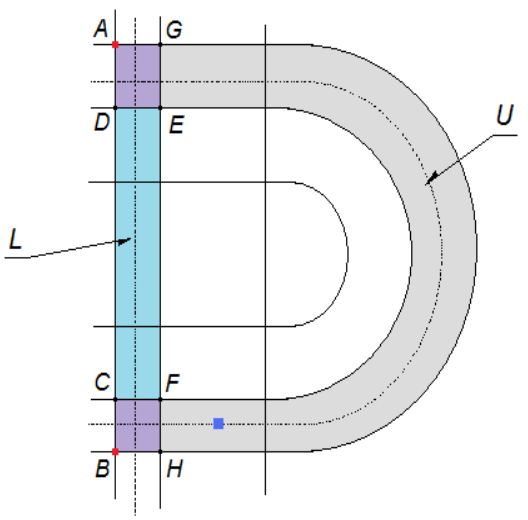

(a) Caso 3 .

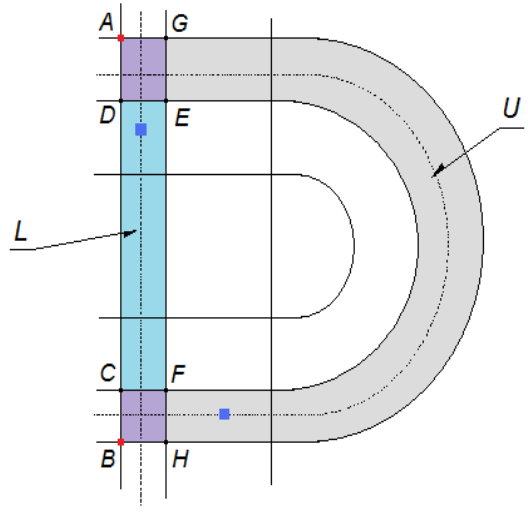

(b) Caso 4 .

Figura 4.6: Discos do tipo periódico periódico-periódico.

Em todos estes caso, se $L$ é atratora para $f$ então $f^{N}(L) \subset L$ ou $f^{2 N}(L) \subset L$; e se $U$ é atratora para $f^{-1}$ então $f^{-N_{1}}(U) \subset U$ o $f^{-2 N_{1}}(U) \subset U$.

\subsubsection{O Teorema de Poda para o derivado da ferradura}

Em todos os casos anteriores podemos definir uma difeotopia, suportada em $D$ que é uma difeotopia de poda. Esta difeotopia é definida por um campo $C^{\infty}$ 
como na seção 3.1.4 seguindo a idea da isotopia de poda: empurrar a dinâmica interessante de $D$ na região livre ou atratora para iterados passados ou futuros. Seja $\mathbb{P}:=\cup_{i \in \mathbb{Z}} f^{k}(D)$, a frente de poda gerada por $D$.

Teorema 4.2.1. Dado um disco de poda homoclínico, existe uma difeotopia $f_{t}$, com $f_{0}=f$, tal que $f_{1}$, o difeo ao final da difeotopia, é um difeo de poda, isto é

i) $\Omega\left(f_{1}\right)=(\Omega(f) \backslash \mathbb{P}) \cup \operatorname{Orb}(p)$ (unido à $\operatorname{Orb}(q)$ quando for o caso),

ii) $\Omega\left(f_{1}\right)$ é hiperbólico tipo sela (exceto em $p$ que é atrator e q que é repulsor).

Demonstração. Faremos a prova para o caso 4 do tipo periódico-periódico. Veja a figura 4.7. Note que a variedade estável $W^{s}(p, F)$ do ponto $p$ pela ferradura $F$ é invariante por $f$. Esta variedade está representada por uma linha vertical pontilhada. O mesmo pode ser dito para a variedade instável $W^{u}(q, F)$ do ponto $q$. Está variedade é a linha horizontal pontilhada. Assim, suponha que o disco de poda $D^{\prime}$ é a região limitada por estas curvas pontilhadas, ou seja, a região definida pelos pontos $A$ e $B$ na figura. Os pontos $p$ e $q$ são os quadrados em azul. Depois utilizamos a construção da isotopia $C^{\infty}$ da seção 3.1.4 no disco $D^{\prime}$.

Podemos ver que todo ponto em $D^{\prime}$ é errante para $f_{1}$ pois ou estão na bacia de atração do ponto $p$ para $f$, ou estão na bacia de atração do ponto $q$ para $f^{-1}$. O mesmo é verdade para os pontos que nunca entram em $D^{\prime}$ e que são errantes para $f$ : Eles estão na bacia de $p$ para $f$, ou na bacia de $q$ para $f^{-1}$, ou convergem a $\infty$ por iterados positivos de $f$ ou convergem a $\infty$ por iterados negativos de $f$. Logo

$$
\Omega\left(f_{1}\right) \subset(\Omega(f) \backslash \mathbb{P}) \cup \operatorname{Orb}(p) \cup \operatorname{Orb}(q) .
$$

Seja $z \in \Omega(f) \backslash \mathbb{P}$, então dada qualquer vizinhança $V$ de $z$, existe um $k_{V}$ tal que $f^{k_{V}}(V) \cap V \neq \emptyset$. Suponha que $V$ é tão pequeno que $D^{\prime} \cap V=\emptyset$. Dado que $z \notin \mathbb{P}$, então para cada $i$ existe um conjunto $M_{i} \subset V$ contendo a $z$ tal que $f^{j}\left(M_{i}\right) \cap D^{\prime}=\emptyset$ para todo $j=0, . ., i$. Assim, existe um $M_{k_{V}} \subset V$ contendo a $z$ tal que $f^{j}\left(M_{k_{V}}\right) \cap D^{\prime}=\emptyset$ para todo $j=0, \ldots, k_{V}$. Então $f^{k_{V}}\left(M_{k_{V}}\right) \cap V \neq \emptyset$. Isto implica que $f_{1}^{k_{V}}\left(M_{k_{V}}\right) \cap V \neq \emptyset$ e portanto $f_{1}^{k_{V}}(V) \cap V \neq \emptyset$. Assim, $z$ é não-errante para $f_{1}$.

Outra maneira de demonstrar que todo ponto em $\Omega(f) \backslash \mathbb{P}$ é não-errante para $f_{1}$ é observando que todo ponto em $\mathbb{P}$ corresponde a uma sequência que possui certos blocos simbólicos de 0's e 1's. Assim, se $z \in \Omega(f) \backslash \mathbb{P}$, a sua sequência simbólica $s(z)=\left(s_{i}\right)_{i \in \mathbb{Z}}$ não possui tais blocos. Logo, podemos construir pontos $f_{1}$-periódicos $z_{n}$ muito próximos de $z$ pegando um bloco $b_{n}$ muito grande de $s(z)$ e repetindo-lo infinitas vezes para formar uma sequência periódica $s^{n}=b_{n}^{\infty}$, tal que $s\left(z_{n}\right)=s^{n}$. Portanto, $z$ está em $\Omega\left(f_{1}\right)$. 


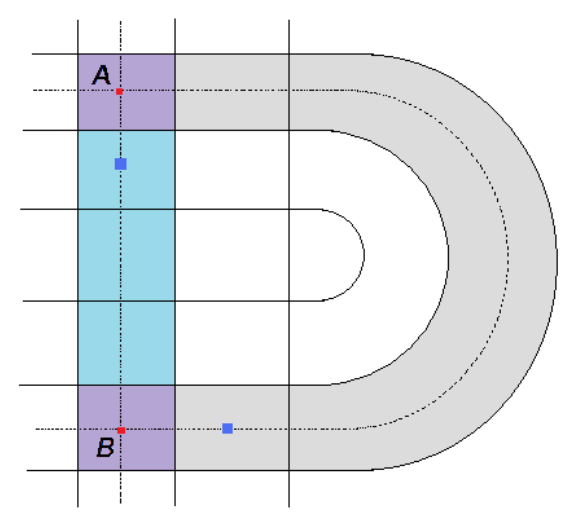

Figura 4.7: Disco de poda.

\subsection{Teoria de poda em superfícies}

Nesta seção faremos a construção de difeomorfismos de poda em superfícies. Depois do feito nas seções anteriores, fica claro qual é o processo a seguir para definir as propriedades dos discos de poda que permitam construir difeos de poda: Encontrar regiões livres e atratoras.

Seja $f: X \rightarrow X$ um difeomorfismo $C^{\infty}$ de uma superfície compacta $X$ e hiperbólico no seu conjunto não errante $\Omega(f)$, o qual está formado por um conjunto tipo sela $\Lambda$ e uma quantidade finita de pontos periódicos atratores e repulsores. Sejam $p$ e $q$ dois pontos selas periódicos de períodos $N_{1}$ e $N_{2}$. Como na seção 4.2, um disco homoclínico $D$ será um domínio simplesmente conexo em $X$ limitado por um segmento da variedade estável de $p, \gamma^{s}(p)$, e um segmento da variedade instável de $q, \gamma^{u}(q)$.

Definição 4.3.1. O disco homoclínico $D$ é um disco de poda se satisfaz as condições da definição 2.3 .3 da seção 2.3 .2 onde $\gamma^{s}(p)$ e $\gamma^{u}(q)$ são os lados $C$ e $E$, ou seja, $D$ satisfaz as condições de [dC99].

Se o segmento $\gamma^{s}(p)$ não tem pontos do conjunto $\Omega(f)$ acumulando-se internamente, então podemos definir uma região livre ou atratora $L$. Se não for o caso, construimos um derivado de $f$, ao qual novamente chamamos de $f$, para abrir o ponto $p$ em três pontos e poder definir a região $L$, de maneira similar ao que fizemos nas seção 4.1 e 4.2 .

O mesmo procedimento anterior pode ser feito para o ponto $q$. Se $\gamma^{u}(q)$ não tem pontos de $\Omega(f)$ acumulando-se internamente então podemos definir a região livre ou atratora $U$ para $f^{-1}$. Se não for o caso, fazemos a construção do derivado de $f$ ao redor do ponto $q$.

Tendo gerado as regiões $L$ e $U$ e tomando coordenadas nas quais $\gamma^{s}(p)$ e $\gamma^{u}(q)$ sejam graficos sobre o eixo $y$, podar o difeomorfismo $f$ significa definir a difeotopia $f_{t}$ como na seção 3.1.4. 
O teorema abaixo, cuja prova será omitida pois segue as ideias que temos utilizado ultimamente, vem a ser o teorema de poda diferenciável para superfícies.

Teorema 4.3.1. Seja $f_{0}$ um difeomorfismo hiperbólico e seja $D$ um disco de poda. Então existe uma difeotopia $f_{t}: f_{0} \simeq f_{1}$ tal que $f_{1}$ é hiperbólico e $\Omega\left(f_{1}\right)=$ $\Omega(f) \backslash \cup_{i \in \mathbb{Z}} f^{k}(D)$.

Se para o difeomorfismo de poda $f_{1}$ podemos encontrar novamente um disco de poda limitado por segmentos de variedades invariantes de dois pontos selas, continuamos podando e construindo derivados do difeomorfismo inicial. Isto permite definir a familia de poda diferenciável $\mathcal{P}(f)$ : começando com um difeo $f$ como na hipôtese do teorema anterior (não apenas a ferradura), podamos repetidas vezes (um número finito de vezes) e tomamos a coleção de todas estas podas. Observe que desta forma obtemos uma construção indutiva: após podarmos uma primeira vez, o nosso procedimento de poda pode ser aplicado novamente e assim indutivamente. 


\section{Parte II}

\section{Aplicações na família de Hénon}




\section{Capítulo 5}

\section{Frentes de poda na família de Hénon}

Neste capítulo apresentamos conexões entre a teoria de poda e a dinâmica na família de Hénon. Na seção 5.1 demonstramos que próximo de transformações de Hénon com entropia maximal, existem difeomorfismos de poda. Isto é possível pois neste caso podemos escolher um disco de poda com diâmetro muito pequeno tal que o difeomorfismo de poda esteja muito próximo da transformação de Hénon na topologia $C^{0}$.

Na seção 5.2 discutimos quais deveriam ser as relações entre a família de Hénon complexa e teoria de poda. Estas estão baseadas nos conceitos que temos introduzido no capítulo 3 e estão relacionadas com certas monodromias no conjunto de ferraduras complexas hiperbólicas denominado o lugar de ferraduras. Assim, sob a condição do teorema 5.2.3, uma transformação de poda pode ser conjugada a um elemento da família de Hénon. Nesta seção também provamos a CFP para alguns parâmetros da família de Hénon utilizando os trabalhos de Zin Arai [Ara07a, Ara07b].

Os discos de poda neste capítulo serão como os discos homoclínicos do capítulo 3.

\subsection{Perturbações de transformações de Hénon}

Em [BS04, BS06b] Eric Bedford e John Smillie estudaram transformações de Hénon com entropia maximal. Uma transformação de Hénon $H_{a_{0}, b_{0}}$ tem entropia maximal se a entropia topologica $h_{t o p}\left(H_{a_{0}, b_{0}}\right)$ é igual a $\log 2$. Eles demonstraram que neste caso ou $H_{a_{0}, b_{0}}$ é hiperbólica e topologicamente conjugada a uma ferradura completa ou possui uma órbita de tangências homoclínicas quadráticas entre as variedades estáveis e instáveis de um ponto fixo a qual ocasiona a perda de hiperbolicidade (Veja [BS04,BS06b]). Da análise das variedades estáveis e instáveis do ponto $0^{\infty}$, Ulrich Hoensch [Hoe08] concluiu que, no segundo caso, a tangência quadrática acontece entre os pontos $0^{\infty} 10 \cdot 10^{\infty}$ e $0^{\infty} 11 \cdot 10^{\infty}$ na codificação canônica da ferradura. Neste caso, a primeira tangência é a primeira bifurcação. Com estes fatos demonstramos o seguinte teorema:

Teorema 5.1.1. Seja $H_{a_{0}, b_{0}}$ uma transformação de Hénon com entropia maximal que não é hiperbólica. Então, existe um $C^{1}$-aberto $\mathcal{U} \neq \emptyset$ em $\operatorname{Diff}^{1}\left(\mathbb{R}^{2}\right)$ tal que: 
(a) dado $\epsilon>0$, existe $f_{\epsilon} \in \mathcal{U}$ tal que $\mathrm{d}_{C^{0}}\left(H_{a_{0}, b_{0}}, f_{\epsilon}\right)<\epsilon$,

(b) se $f \in \mathcal{U}$ então $\Omega(f)$ é hiperbólico e existe um disco de poda $D_{f}$ tal que fé topologicamente conjugado ao difeomorfismo de poda $H_{D_{f}}$ associado a $D_{f}$ como no teorema de poda quando ambos estão restritos aos seus conjuntos não errantes.

Demonstração. Seja $H_{a_{0}, b_{0}}$ uma transformação de Hénon com entropia maximal que não é hiperbólica. Pelo teorema 2.2 .4 e a proposição $2.2 .5, H_{a_{0}, b_{0}}$ possui uma tangência homoclínica nos pontos $0^{\infty} 10 \cdot 10^{\infty}$ e $0^{\infty} 11 \cdot 10^{\infty}$. Dado $\epsilon>$ 0 , é suficiente mostrar que existem pontos homoclínicos $p_{0}$ e $p_{1}$, próximos da tangência $t:=0^{\infty} 10 \cdot 10^{\infty}=0^{\infty} 11 \cdot 10^{\infty}$, definindo um disco de poda $D$ tal que $\operatorname{diam}(H(D))<\epsilon$.

Por 2.2.5, se $\mathcal{T}:=\operatorname{Orb}(t)$ denota o conjunto de tangências homoclínicas entre $W^{u}\left(K_{a, b}\right)$ e $W^{s}\left(K_{a, b}\right)$ então $K_{a, b}$ é totalmente desconexo numa vizinhança de cada ponto de $K_{a, b} \backslash \mathcal{T}$ e estas desconexões ocorrem em órbitas homoclínicas ao $0^{\infty}$, então é possível construir discos homoclínicos que passam por órbitas homoclínicas. Observe que as propriedades de poda não são modificadas se existe uma tangência homoclínica entre os pontos $0^{\infty} 10 \cdot 10^{\infty}$ e $0^{\infty} 11 \cdot 10^{\infty}$. Tome $N, M \in \mathbb{N}$ suficientemente grandes tal que os pontos $p_{0}:=0^{\infty} 110^{N} 11$. $10^{M} 110^{\infty}$ e $p_{1}:=0^{\infty} 110^{N} 10 \cdot 10^{M} 110^{\infty}$ definen um disco de poda $D_{N, M}$ que satisfaz $\operatorname{diam}\left(H\left(D_{N, M}\right)\right)<\epsilon$. Use a construção da seção 3.1.4 para construir um difeomorfismo de poda $H_{1}$ como no teorema de poda associado a $D_{N, M}$. Logo, $d_{C^{0}}\left(H_{1}, H\right)<\epsilon$. Assim, pela proposição 3.1.7, $H_{1}$ é hiperbólico no conjunto $\Omega\left(H_{1}\right)$. Pelo teorema 2.1.2 existe uma $C^{1}$-vizinhança $\mathcal{U}_{1}$ de $H_{1}$ tal que para cada $g$ em $\mathcal{U}_{1}$ existe um conjunto $g$-invariante $\Lambda^{\prime}$ tal que $\left.g\right|_{\Lambda^{\prime}}$ é topologicamente equivalente a $\left.H_{1}\right|_{\Omega\left(H_{1}\right)}$. A vizinhança $\mathcal{U}_{1}$ é escolhida de maneira que cada $g \in \mathcal{U}_{1}$ tem como conjunto não errante a $\Lambda^{\prime}$.

Repetindo a construção do paragrafo anterior para todos os discos de poda $D$ cujos pontos homoclínicos estão suficientemente próximos da tangência, podemos afirmar que existe uma transformação de poda $H_{D} \in \operatorname{Diff}^{\infty}\left(\mathbb{R}^{2}\right)$ suficientemente próxima de $H_{a_{0}, b_{0}}$ e uma $C^{1}$-vizinhança $\mathcal{U}_{D}$ de $H_{D}$ tal que cada $g \in \mathcal{U}_{D}$ é topologicamente conjugado a $H_{D}$ quando ambos estão restritos a seus conjuntos não errantes. Portanto, se definirmos

$$
\mathcal{U}:=\bigcup_{D} \mathcal{U}_{D}
$$

temos que $\mathcal{U}$ satisfaz as conclusões do teorema.

\subsection{Frentes de poda na família de Hénon complexa}

A família de Hénon

$$
H_{a, b}(x, y)=\left(a-x^{2}-b y, x\right)
$$

tem uma extensão natural a $\mathbb{C}^{2}$ considerando $H_{a, b}: \mathbb{C}^{2} \rightarrow \mathbb{C}^{2} \operatorname{com}(a, b) \in \mathbb{C} \times \mathbb{C}^{*}$, a qual tem sido muito utilizada por vários autores, especialmente por Bedford 
e Smillie [BS04, BS06b], Hubbard e Oberste-Vorth [HOV95, HOV94], Fornaes e Sibony [FS92]. Uma maneira de estudar a dinâmica de uma transformação de Hénon é ver qual é a topologia do lugar de ferraduras, que é conjunto de parâmetros $(a, b) \in \mathbb{C}^{2}$ tais que o conjunto de órbitas limitadas de $H_{a, b}$ constitui uma ferradura complexa hiperbólica completa (veja [BS06a]).

Nesta seção observaremos algumas relações entre a teoria de poda e o lugar de ferraduras. Estas são uma consequência do trabalho de Arai [Ara07b] sobre monodromias no lugar de ferraduras complexas e nosso trabalho prévio sobre a teoria de poda desenvolvido no capítulo 3. Apresentamos, no teorema 5.2.3, uma condição que implica que uma transformação de Hénon é uma transformação de poda da ferradura e que permite demonstrar a CFP em alguns valores do plano de parâmetros.

\subsubsection{O lugar de ferraduras}

Utilizaremos as notações do artigo de Arai [Ara07b]. Seja $K_{a, b}^{\mathbb{C}}$ o conjunto de órbitas limitadas

$$
K_{a, b}^{\mathbb{C}}=\left\{p \in \mathbb{C}^{2} \mid\left\{H_{a, b}^{n}(p)\right\}_{n \in \mathbb{Z}} \text { é limitada }\right\}
$$

e $K_{a, b}^{\mathbb{R}}:=K_{a, b}^{\mathbb{C}} \cap \mathbb{R}^{2}$ a sua interseção com $\mathbb{R}^{2}$. Seja $\mathcal{H}^{\mathbb{C}}$ o conjunto de parâmetros $(a, b) \in \mathbb{C}^{2}$ tais que a restrição de $H_{a, b}$ a $K_{a, b}^{\mathbb{C}}$ é topologicamente conjugada ao 2-shift $\left(\sigma, \Sigma_{2}\right)$. $\mathcal{H}^{\mathbb{C}}$ é chamado o lugar de ferraduras. De modo similar, seja $\mathcal{H}^{\mathbb{R}}$ o conjunto de parâmetros $(a, b) \in \mathbb{R}^{2}$ tais que a restrição de $H_{a, b}$ a $K_{a, b}^{\mathbb{R}}$ é topologicamente conjugado ao 2-shift.

Devaney e Nitecki demonstraram que $\mathcal{H}^{\mathbb{R}} \neq \emptyset$ e que contém o conjunto $\mathrm{DN}:=\left\{(a, b) \in \mathbb{R}^{2} \mid a>(5+2 \sqrt{5})(1+|b|)^{2} / 4, b \neq 0\right\} ;$ além disso, eles mostraram que se $(a, b) \in \mathrm{EMP}:=\left\{(a, b) \in \mathbb{R}^{2}: a<-(1+|b|)^{2} / 4\right\}$ então $K_{a, b}^{\mathbb{R}}=\emptyset$ (veja [DN79]).

Hubbard e Oberste-Vorth demonstraram que $\mathcal{H}^{\mathbb{C}}$ contém o conjunto HOV $:=$ $\left\{(a, b) \in \mathbb{C}^{2}:|a|>2(1+|b|)^{2}, b \neq 0\right\}($ veja $[\mathrm{OV} 87])$. Note que DN $\subset$ HOV.

Em $\left[\right.$ Ara07b], Arai demonstrou que existem parâmetros $(a, b) \in \mathcal{H}^{\mathbb{C}} \cap \mathbb{R}^{2}$ nos quais $K^{\mathbb{R}} \neq \emptyset$ mas $H_{a, b}$ restrito a $K^{\mathbb{R}}$ não é conjugado ao 2 -shift. Estes parâmetros foram chamados parâmetros de tipo-3 (Veja [BS06a]). Também em [BS06a], Bedford e Smillie exibiram uma maneira de conectar distintas componentes conexas do lugar real de ferraduras, $\mathcal{H}^{\mathbb{C}} \cap \mathbb{R}^{2}$, usando laços em $\mathcal{H}^{\mathbb{C}}$ que começam em DN. Seja $\mathcal{H}_{0}^{\mathbb{C}}$ a componente conexa de $\mathcal{H}^{\mathbb{C}}$ que contém HOV e tome um ponto $\left(a_{0}, b_{0}\right) \in \mathrm{DN} \subset \mathcal{H}_{0}^{\mathbb{C}}$. Então existe uma conjugação canônica

$$
g_{0}: K_{a_{0}, b_{0}}^{\mathbb{C}} \rightarrow \Sigma_{2}
$$

entre $H_{a_{0}, b_{0}}$ e $\sigma$. Seja $\gamma(t)$ um laço em $\mathcal{H}_{0}^{\mathbb{C}}$ com ponto base em $\left(a_{0}, b_{0}\right)$. Pela estabilidade estrutural existem conjugações $g_{t}$ entre $K_{\gamma(t)}^{\mathbb{C}}$ e $\Sigma_{2}$. Dado que $\gamma(0)=\gamma(1)=\left(a_{0}, b_{0}\right)$, podemos definir um automorfismo do shift

$$
\rho(\gamma):=g_{1} \circ g_{0}^{-1} \in \operatorname{Aut}\left(\Sigma_{2}\right)
$$


onde $\operatorname{Aut}\left(\Sigma_{2}\right)$ é o grupo de automorfismos de $\Sigma_{2}$ que consiste de todos os homeomorfismos de $\Sigma_{2}$ em $\Sigma_{2}$ que comutam com $\sigma$. Assim, $\rho$ leva um laço $\gamma$ em um automorfismo em $\operatorname{Aut}\left(\Sigma_{2}\right)$ o qual só depende da classe de homotopia do laço, $[\gamma]$. Portanto, a aplicação:

$$
\rho: \pi_{1}\left(\mathcal{H}_{0}^{\mathbb{C}},\left(a_{0}, b_{0}\right)\right) \rightarrow \operatorname{Aut}\left(\Sigma_{2}\right)
$$

dada por $[\gamma] \rightarrow \rho(\gamma)$ é um homomorfismo de grupos bem definido chamado homomorfismo de monodromia.

Assim, podemos enunciar a Conjectura de Hubbard:

Conjectura de Hubbard: $\rho$ é sobrejetor.

Bedford e Smillie também demonstraram que se $\gamma$ representa um elemento de $\pi_{1}(\mathrm{HOV})$ então ou $\rho(\gamma)$ é a identidade ou $\rho(\gamma)$ é o automorfismo de $\Sigma_{2}$ que substitui os simbolos 0 e 1, um pelo outro [BS06a]. Recentemente, Arai [Ara07b] demonstrou, usando uma demonstração numérica rigorosa, que $\Gamma$ tem um elemento de ordem infinita.

O resultado principal de Arai [Ara07b] está relacionado a laços em $\mathcal{H}_{0}^{\mathbb{C}}$ que passam através de parâmetros reais $(a, b)$. Sua formulação é

Teorema 5.2.1 (Arai). Se $(a, b) \in \mathcal{H}_{0}^{\mathbb{C}} \cap \mathbb{R}^{2}$, então existe um laço $\gamma:=\bar{\alpha}^{-1} \cdot \alpha$ com $\gamma(1 / 2)=(a, b)$, onde $\alpha$ é um caminho em $\mathcal{H}_{0}^{\mathbb{C}}$ com extremos em $\left(a_{0}, b_{0}\right) e$ $(a, b)$, tal que o seguinte diagrama comuta:

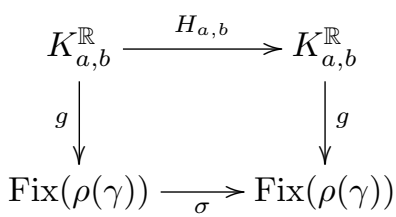

onde $g_{1 / 2}$ é a conjugação entre $K_{a, b}^{\mathbb{C}}$ e $\Sigma_{2}, g:=\left.g_{1 / 2}\right|_{K_{a, b}^{\mathbb{R}}}$ e $\operatorname{Fix}(\rho(\gamma))$ é o conjunto de pontos fixos de $\rho(\gamma)$.

Uma interessante questão é a seguinte: Que automorfismos no teorema anterior são realizáveis por transformações de Hénon no lugar real de ferraduras? Na seguinte seção, observaremos que estes automorfismos podem ser associados a automorfismos definidos por transformações de poda.

\subsubsection{Frentes de poda e curvas em $\mathcal{H}_{0}^{\mathbb{C}}$}

Precisaremos algumas definições do capítulo 3 onde construímos os difeomorfismos de poda da ferradura de Smale $F$ definida por uma transformação de Hénon $H$.

Dado $N \in \mathbb{N}$, defina $s_{i}^{N}:=\underbrace{s_{i} s_{i} \ldots s_{i}}_{N-\text { vezes }}$ onde $s_{i}^{0}=\emptyset$ é a sequência vazia. Para o que estamos interessados aqui, um disco de poda será um disco $D_{N, M}$ tal que $H^{-1}\left(D_{N, M}\right)$ contém os pontos correspondentes a os blocos das sequências simbólicas $0^{N} 1 \cdot 010^{M}$ e $0^{N} 1 \cdot 110^{M}$. Então $D_{N, M}$ é limitado por segmentos 
de variedades estáveis e instáveis que passam pelos pontos homoclínicos $p_{0}=$ $0^{\infty} 110^{N-1} 11 \cdot 10^{M-1} 110^{\infty}$ e $p_{1}=0^{\infty} 110^{N-1} 10 \cdot 10^{M-1} 110^{\infty}$. A seguinte proposição dá-nos condições nas quais o disco $D_{N, M}$ é um disco de poda,

Proposição 5.2.1. $D_{N, M}$ é um disco de poda homoclínico, se $N, M \geq 3$. Além disso, o mesmo vale nos seguintes casos:

(i) $N=0$ e $M \geq 2$, ou $M=0$ e $N \geq 2$,

(ii) $N=1$ e $M \geq 2$, ou $M=1$ e $N \geq 2$,

(iii) $N=2$ e $M \geq 1$, ou $M=2$ e $N \geq 1$.

Seja $f_{N, M}$ o difeomorfismo de poda associado ao disco $D_{N, M}$ como no Teorema de poda. O seguinte resultado, que será crucial para nossas observações, afirma que para cada $f_{N, M}$ existe um automorfismo que depende unicamente dos pontos homoclínicos que definirem o disco $D_{N, M}$ :

Teorema 5.2.2. Para cada disco $D_{N, M}$ como na proposição 5.2.1, então existe um automorfismo do shift $\rho_{N, M} \in \operatorname{Aut}\left(\Sigma_{2}\right)$ tal que o seguinte diagrama comuta:

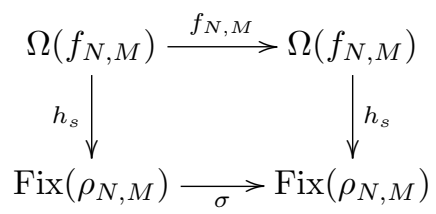

onde $h_{s}$ é a restrição de $h$ ao conjunto não errante de $f_{N, M}, \Omega\left(f_{N, M}\right)$.

Demonstração. Considere o automorfismo $\rho_{N, M}$ que substitui as sequências $0^{N} 1010^{M}$ e $0^{N} 1110^{M}$, una pela outra:

$$
\left(\rho_{N, M}(s)\right)_{i}=\left\{\begin{array}{cc}
0 & \text { se } s_{i-N-1} \ldots s_{i} \ldots s_{i+M+1}=0^{N} 1110^{M} \\
1 & \text { se } s_{i-N-1} \ldots s_{i} \ldots s_{i+M+1}=0^{N} 1010^{M} \\
s_{i} & \text { em outro caso }
\end{array}\right.
$$

Note que

$$
\mathbb{P}_{s}=\left\{s \in \Sigma_{2} \mid \exists i \in \mathbb{Z} \text { tal que } s_{i-1-N} . . s_{i} . . s_{i+1+M} \in\left\{0^{N} 1010^{M}, 0^{N} 1110^{M}\right\}\right\}
$$

onde $\mathbb{P}_{s}$ é a frente de poda simbólica. Então $\Sigma_{2} \backslash \mathbb{P}_{s}=\operatorname{Fix}\left(\rho_{N, M}\right)$. Assim, pelo lema 3.1.2, $h_{s}$ é uma conjugação entre $f_{N, M}$ restrito a $\Omega(H) \backslash \mathbb{P}$ e o shift $\sigma$ restrito a $\operatorname{Fix}\left(\rho_{N, M}\right)$, onde $\mathbb{P}$ é a frente de poda associada a $D_{N, M}$. Como, pelo teorema de poda, $\Omega\left(f_{N, M}\right)=\Omega(H) \backslash \mathbb{P}$, o teorema segue.

É claro que o teorema 5.2.2 pode ser generalizado a todo disco de poda e a dois ou mais discos de poda. O automorfismo do shift definido pelo disco $D_{N, M}$ como na prova do teorema anterior será chamado automorfismo de poda associado ao disco $D_{N, M}$. 
Definição 5.2.1. Seja $\gamma$ uma curva fechada em $\mathbb{C}^{2}$ e $\bar{\gamma}:=\varphi(\gamma)$ onde $\varphi(x, y)=$ $(\bar{x}, \bar{y})$ é a conjugação complexa. Dizemos que $\gamma$ é simétrica se $\bar{\gamma}=\gamma^{-1}$, isto é, $\bar{\gamma}(t)=\gamma(1-t), \forall t \in[0,1]$. Note que quando $\gamma$ é simétrica temos que $\gamma(0), \gamma(1 / 2) \in \mathbb{R}^{2}$. Doravante vamos supor que $\gamma(0) \in D N$.

O teorema 5.2.2 mostra uma conexão entre uma poda da ferradura de Smale e as possíveis dinâmicas reais de $H_{a, b}$ quando $(a, b) \in \mathcal{H}_{0}^{\mathbb{C}} \cap \mathbb{R}^{2}$ no seguinte sentido:

Teorema 5.2.3. Seja $\rho_{N, M}$ o automorfismo de poda associado ao disco $D_{N, M}$. Suponha que existe uma curva $\gamma \in \mathcal{H}_{0}^{\mathbb{C}}$ simétrica tal que $\rho(\gamma)=\rho_{N, M}$ e seja $(a, b)_{N, M}=\gamma(1 / 2)$. Então existe uma conjugação entre a transformação de Hénon $H_{(a, b)_{N, M}}$ restrita a $K_{(a, b)_{N, M}}^{\mathbb{R}}$ e a transformação de poda $f_{N, M}$ restrita $a \Omega\left(f_{N, M}\right)$.

Demonstração. Seja $g_{t}$ as conjugações entre $H_{\gamma(t)}$ e $\sigma$ restritos a $K_{\gamma(t)}^{\mathbb{C}}$ e $\Sigma_{2}$ respectivamente. Então $\rho_{N, M}=g_{1} \circ\left(g_{0}\right)^{-1}$ por definição. Seja $\alpha:=\left.\gamma\right|_{[0,0.5]}$, então $\gamma=\bar{\alpha}^{-1} \cdot \alpha$ e pelo teorema 5.2.1 de Arai, $g_{1 / 2}$ é uma conjugação entre $H_{a, b}$ e $\sigma$ restritos a $K_{a, b}^{\mathbb{R}}$ e $\operatorname{Fix}\left(\rho_{N, M}\right)$, respectivamente. Então pelo teorema 5.2 .2 a conjugação procurada é $\left(h_{s}\right)^{-1} \circ g_{1 / 2}$.

Note que se a conjectura de Hubbard é verdadeira então para cada automorfismo de poda $\rho_{N, M}$ existirá um laço não trivial $\gamma_{N, M}$ em $\mathcal{H}^{\mathbb{C}}$. Se, além disso, $\gamma_{N, M}$ passa por parâmetros reais $(a, b)$, então, pelo teorema 5.2 .3 , a dinâmica real de $H_{a, b}$ será conjugada à dinâmica do difeomorfismo de poda $f_{N, M}$. Logo, a análise de quais difeomorfismos de poda existem na família de Hénon poderia trazer conhecimento sobre qual é a topologia do lugar real de ferraduras.

Como uma consequência do teorema 5.2.3 obtemos uma prova da CFP para alguns valores dos parâmetros:

Teorema 5.2.4. Há dois conjuntos abertos disjuntos $A_{1}$ e $A_{2}$ no plano de parâmetros reais tal que se $(a, b) \in A_{1} \cup A_{2}$ então $H_{a, b}$ é topologicamente conjugado a uma poda não trivial da ferradura.

Demonstração. Considere os parâmetros $(a, b) \in[5.3125,5.46875] \times\{1\}$. Por [Ara07a], estes parâmetros correspondem a transformações de Hénon hiperbólicas. Então existe um conjunto aberto conexo $A_{1}$ tal que todas as transformações ali são conjugadas. Por [Ara07b], existe uma curva simétrica $\gamma_{1} \in \mathcal{H}_{0}^{\mathbb{C}}$ tal que $\gamma_{1}(1 / 2)=(a, b)$ e $\rho\left(\gamma_{1}\right)=\rho_{2,2}$. Então, pelo teorema $5.2 .3, H_{a, b}$, restrito a $K_{a, b}^{\mathbb{R}}$, é conjugado ao difeomorfismo de poda $f_{2,2}$ associado ao disco de poda $D_{2,2}$ e restrito a $\Omega\left(f_{2,2}\right)$.

O aberto $A_{2}$ é obtido considerando os valores $(a, b) \in[2.21875,2.296875] \times$ $\{0.25\}$. A prova segue o mesmo raciocinio. Neste caso $H_{a, b}$, restrito a $K_{a, b}^{\mathbb{R}}$, é conjugado ao difeomorfismo de poda $f_{0,2}$ associado ao disco de poda $D_{0,2}$ e restrito a $\Omega\left(f_{0,2}\right)$.

Observação 5.2.1. Pelo algoritmo da seção 3.2 é fácil construir as partições de Markov para as transformações de Hénon do teorema anterior (Compare 
com [DMS91] quando $(a, b)=(5.4,1))$. Para os valores no conjunto $A_{1}$ a matriz é:

$$
A_{1}=\left[\begin{array}{llllllll}
1 & 1 & 1 & 0 & 0 & 0 & 0 & 0 \\
0 & 0 & 0 & 1 & 1 & 0 & 0 & 0 \\
0 & 0 & 0 & 0 & 0 & 1 & 0 & 0 \\
0 & 0 & 0 & 0 & 0 & 1 & 0 & 0 \\
0 & 0 & 0 & 0 & 0 & 0 & 1 & 1 \\
1 & 0 & 0 & 0 & 0 & 0 & 0 & 0 \\
0 & 0 & 1 & 1 & 0 & 0 & 1 & 1 \\
0 & 0 & 0 & 1 & 0 & 0 & 1 & 0
\end{array}\right]
$$

Para valores no conjunto $A_{2}$, a matriz é:

$$
A_{2}=\left[\begin{array}{llllll}
1 & 1 & 1 & 0 & 0 & 0 \\
0 & 0 & 0 & 1 & 1 & 0 \\
0 & 0 & 0 & 0 & 0 & 1 \\
0 & 0 & 0 & 0 & 0 & 1 \\
0 & 1 & 0 & 0 & 1 & 0 \\
1 & 0 & 0 & 0 & 0 & 0
\end{array}\right]
$$

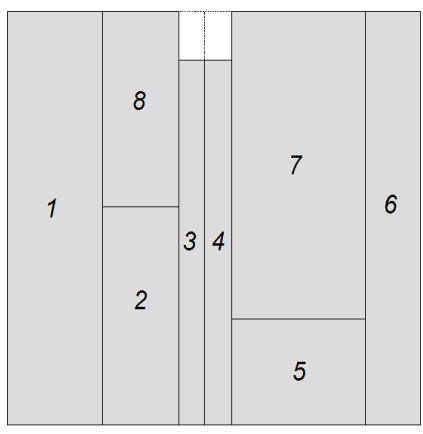

(a) Usando o disco $D_{2,2}$.

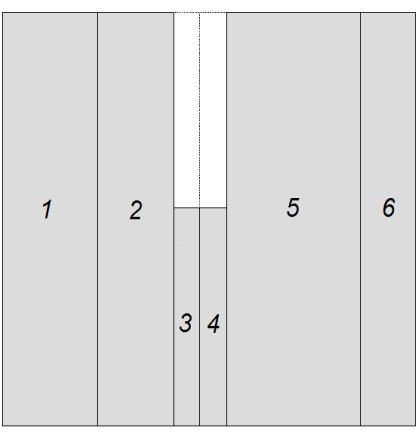

(b) Usando o disco $D_{0,2}$.

Figura 5.1: Partições de Markov para os valores no conjunto $A_{1}$ (figura 5.1(a)) e para os valores no conjunto $A_{2}$ (figura 5.1(b)) do teorema 5.2.4. 


\section{Capítulo 6}

\section{Problemas de pesquisa}

Neste capítulo reportamos alguns problemas de pesquisa que se deduzem dos capítulos anteriores relacionando a teoria de poda e a dinâmica na família de Hénon.

Na seção 6.1 estudamos a família de Hénon real quando esta preserva área e analisamos o tipo de subshift que deve estar associado mediante poda. $\mathrm{Na}$ seção 6.2 mostramos mais evidência numérica que suporta a CFP. Na seção 6.3 observarmos que, pelo menos numericamente, estes valores estão no lugar de ferraduras. Na seção 6.4 apresentamos uma conjectura relacionando as construções de poda com o lugar de ferraduras.

\subsection{Quando $H_{a, b}$ preserva área}

O caso $b=1$, o qual corresponde a transformações de Hénon que preservam orientação e área, tem sido muito estudado. No ano 1991, Davis, Mackay e Sannami [DMS91] conjecturaram que, quando $a=5.4, a=5.59$ e $a=5.65, H_{a, 1}$ é hiperbólico e exibiram um subshift de tipo finito para a dinâmica do conjunto não errante em cada um destes valores. Estes subshifts provinham, conjecturalmente, de uma região na qual as variedades invariantes foram descruzadas. Em 2002, de Carvalho and Hall em [dCH02b] replicaram estes resultados para $a=5.4$ usando o abordagem da teoria de poda. Eles associaram conjecturalmente um subshift de tipo finito a $H_{5.4,1}$ em termos de uma frente de poda. Este é a frente de poda da figura 1.2(b). Finalmente no 2007, Arai em [Ara07b] demonstrou que $H_{5.4,1}$ restrita ao seu conjunto não errante é, de fato, hiperbólica utilizando rigorosas pesquisas numéricas. Hoje, pelo nosso teorema de poda, o resultado de Arai se pode interpretar como uma poda da ferradura utilizando um disco de poda simétrico.

Gostariamos de fazer algumas observações sobre como devem estar relacionadas a teoria de poda e a dinâmica de trasformações de Hénon que preservam área. Destas observações parece-se inferir que se uma destas transformações é uma poda então o disco de poda associado é um disco simétrico. Isto segue utilizando a reversibilidade do shift $\sigma$ em $\Sigma_{2}$. Antes precisamos de algumas definições.

Definição 6.1.1. Seja $X$ um espaço topológico e $f: X \rightarrow X$ um homeomorfismo. Um homeomorfismo $g: X \rightarrow X$ é chamado um reversor para $(X, f)$ 
se

$$
g \circ f=f^{-1} \circ g
$$

e $g^{2}=I d$. Dizemos que $(X, f)$ é um sistema reversível se admite um reversor $g$ e, então, $(X, f, g)$ é um sistema reversor.

É claro que $\left(\Sigma_{2}, \sigma\right)$ é reversível pelo reversor

$$
\phi: \Sigma_{2} \rightarrow \Sigma_{2}
$$

definido por $\phi\left(s_{-} \cdot s_{+}\right)=s_{+} \cdot s_{-}$, isto é, $(\phi(s))_{i}=s_{-(i+1)}$.

Seja $T: \mathbb{R}^{2} \rightarrow \mathbb{R}^{2}$ a transformação $T(x, y)=(y, x)$. Se $H_{a, b}$ preserva área, $b=1$, então

$$
T \circ H_{a, 1}(x, y)=H_{a, 1}^{-1} \circ T(x, y)
$$

Assim $\left(\Omega\left(H_{a, 1}\right), H_{a, 1}\right)$ é um sistema reversível.

Definição 6.1.2. Dizemos que um disco de poda $D$, definido pelos pontos homoclínicos $p_{0}$ e $p_{1}$ é simétrico se

$$
\phi\left(\sigma^{-1}\left(p_{k}\right)\right)=p_{k}, \text { para } i=1,2 .
$$

Observe que se um disco de poda $D$ é simétrico então é definido pelas órbitas homoclínicas $p_{0}=0^{\infty} 1 \mathrm{w}^{*} 1 \cdot \mathrm{w} 10^{\infty}$ e $p_{1}=0^{\infty} 1 \mathrm{w}^{*} 0 \cdot \mathrm{w} 10^{\infty}$ onde w é uma sequência finita de símbolos 0 e 1 tal que a soma de símbolos é par e, se $\mathrm{w}=w_{1} w_{2} \ldots w_{n}$ então $\mathrm{w}^{*}=w_{n} w_{n-1} \ldots w_{1}$.

Proposição 6.1.1. Seja $D$ um disco de poda simétrico e seja $D_{s}:=h(D \cap$ $\Omega(H)$ ). Então:

$$
s \in D_{s} \text { se, e somente se, } \phi\left(\sigma^{-1}(s)\right) \in D_{s} .
$$

Demonstração. Seja $s=\ldots s_{-1} \cdot s_{0} s_{1} \ldots$ Lembre que a codificação canônica preserva a ordem unimodal nas projeções, assim $s \in D_{s}$ se e somente se

$$
\mathrm{w} 10^{\infty}<s_{0} s_{1} \ldots \leq 10^{\infty} \text { e } 0 \mathrm{w} 10^{\infty}<s_{-1} s_{-2} \ldots<1 \mathrm{w} 10^{\infty} .
$$

Mas, pela dinâmica do $\sigma$ (considerando-o como uma transformação unimodal do intervalo), estas condições são equivalentes as seguintes

$$
\text { w1 } 0^{\infty}<s_{-2} s_{-3} \cdots \leq 10^{\infty} \text { e } 0 \mathrm{w} 10^{\infty}<s_{-1} s_{0} \ldots<1 \mathrm{w} 10^{\infty} .
$$

Estas últimas condições são equivalentes a dizer que $\phi\left(\sigma^{-1}(s)\right) \in D_{s}$.

Seja $\left(\Sigma_{A}, \sigma_{A}\right)$ o subshift obtido no Teorema 1.3.1. Com estas definições podemos provar as seguintes

Proposição 6.1.2. Se D é um disco de poda simétrico então o subshift $\left(\Sigma_{A}, \sigma_{A}\right)$ é reversivel. 
Demonstração. Pela proposição 6.1.1, um disco de poda simétrico satisfaz: $s \in$ $D_{s}$ se, e somente se, $\phi\left(\sigma^{-1}(s)\right) \in D_{s}$. Pela equação $\sigma \circ \phi=\phi \circ \sigma^{-1}$ temos que $s \in \sigma^{n}\left(D_{s}\right)$ se, e somente se, $s \in \phi\left(\sigma^{-n}\left(\phi\left(D_{s}\right)\right)\right)$ se e somente se $\phi(s) \in$ $\sigma^{-(n+1)}\left(\sigma\left(\phi\left(D_{s}\right)\right)\right)$ se e somente se $\phi(s) \in \sigma^{-(n+1)}\left(D_{s}\right)$. Por tanto $s \in \mathbb{P}_{s}$ se e somente se $\phi(s) \in \mathbb{P}_{s}$. Logo

$$
\phi_{A}: \Sigma_{2} \backslash \mathbb{P}_{s} \rightarrow \Sigma_{2} \backslash \mathbb{P}_{s}
$$

definido por $\phi_{A}=\left.\phi\right|_{\Sigma_{2} \backslash \mathbb{P}_{s}}$ é um homeomorfismo bem definido. Lembremos a transformação $\psi: \Sigma_{A} \rightarrow \Sigma_{2}$ dada na definição 3.3.1. Sabemos que $\psi\left(\Sigma_{A}\right)=$ $\Sigma_{2} \backslash \mathbb{P}_{s}$. Assim, $\psi$ é sobrejetora. Suponha que $\psi(a)=\psi\left(a^{\prime}\right)$. Então pelo teorema 1.3.1, existe $x, y \in \Omega\left(H_{1}\right)$ tal que $\tau(x)=a$ e $\tau(y)=a^{\prime}$. Logo $\psi(\tau(x))=\psi(\tau(y))$ o que implica que $h_{s}(x)=h_{s}(y)$ ou $x=y$ dado que $h_{s}$ é injetiva. Por tanto $a=$ $a^{\prime}$ e assim $\psi$ é injetiva. Note que $\psi$ é continua. Então $\psi$ é um homeomorfismos entre $\Sigma_{A}$ e $\Sigma_{2} \backslash \mathbb{P}_{s}$. Se definirmos $\hat{\phi}: \Sigma_{A} \rightarrow \Sigma_{A}$ por $\hat{\phi}(a)=\psi^{-1} \phi_{A} \psi(a)$ então $\hat{\phi}$ é um reversor para o sistema $\left(\Sigma_{A}, \sigma_{A}\right)$.

Proposição 6.1.3. Se $\left(\Omega\left(H_{a, 1}\right), H_{a, 1}\right)$ é conjugado ao difeomorfismo de poda obtido utilizando um disco $D$ então o sistema $\left(\Sigma_{A}, \sigma_{A}\right)$ é reversível.

Demonstração. Seja $f: \Omega\left(H_{a, 1}\right) \rightarrow \Omega\left(H_{1}\right)$ a conjugação entre $H_{a, 1}$ e a transformação de poda $H_{1}$ associada ao disco $D$. Lembre que $\tau$ é uma conjugação entre $H_{1}$ e $\sigma_{A}$. Então $\tilde{\phi}:=\tau f T f^{-1} \tau^{-1}$ é um reversor para o sistema $\left(\Sigma_{A}, \sigma_{A}\right)$.

As duas proposições anteriores indicam que reversibilidade e simetria são conceitos ligados. Podemos então perguntar:

Qual é a relação entre $\tilde{\phi}$ e $\hat{\phi}$ ?

Se eles são iguais então poderiamos provar que se uma trasformação de Hénon que preserva área é um difeomorfismo de poda então o disco de poda associado é simétrico. Em outras palavras, quando $b=1$ só alguns tipos de poda seriam permitidos. Isto motiva as seguintes questões:

Para $b$ fixo, que tipos de poda são permitidos?

Note que os discos $D_{N, N}$ são simétricos. Assim eles corresponderiam a podas reversíveis. Logo uma pergunta natural é:

Se $H_{a, b}$ é uma poda associada ao disco $D_{N, M}$, qual é a relação entre $M, N$ e $b ?$

\subsection{Evidência numérica}

Nós temos realizado experimentos numéricos com a família de Hénon e temos encontrado parâmetros onde uma transformação de Hénon pode ser interpretada como um difeomorfismo de poda. Pelo trabalho de Arai [Ara07a], sabemos que muitos destes parâmetros correspondem a parâmetros onde a transformação de Hénon é hiperbólica. Note que entre estes parâmetros estão aqueles estudados por Davis, Mackay and Sannami em [DMS91] e usamos a uma ideia destes últimos autores para conjecturar que certas transformações de Hénon são difeomorfismos de poda, isto é: achamos numericamente órbitas periódicas de período pequeno pelo método de Biham-Wenzel [BW89] e comparamos com 
o número de órbitas periódicas do subshift associado ao disco de poda ou à coleção de discos poda.

As podas que temos contruido para estes exemplos são definidas por um ou dois discos de poda. No caso de dois discos de poda, utilizamos duas vezes o teorema de poda diferenciável. Embora não apresentamos construções de algoritmo usando dois ou mais discos de poda, é claro que este algoritmo se pode generalizar a varios discos homoclínicos acrescentando algumas condições técnicas.

Observe que o shift de Markov do difeomorfismo de poda conjecturado para a transformação de Hénon do exemplo 6.2.1 possui a mesma entropia topologica que a limite inferior encontrada pelo Rafael Frongillo em [Fro10]. Dos exemplos 6.2.2 ao 6.2.5, a entropia topologica do shift coincide com as estimativas numéricas feitas por Hagiwara e Shudo em [HS04].

Para encontrar todos estes exemplos temos utilizado um programa para desenhar as variedades invariantes ao ponto $0^{\infty}$ que foi criado por Toby Hall. Os valores dos parâmetros estão discriminados nas seguintes subseções.

Lembre a definição dos discos $D_{N, M}$ da proposição 5.2.1.

\subsubsection{Usando um disco de poda}

Exemplo 6.2.1. Para $H_{3.5197,0.648}$ conjecturamos que o disco de poda é $D_{1,2}$. Pelo algoritmo, construimos a sua partição de Markov (Figure 6.1(a)). A matriz de transição $A_{1}$ é a seguinte:

$$
A_{1}=\left[\begin{array}{lllllll}
1 & 1 & 1 & 0 & 0 & 0 & 0 \\
0 & 0 & 0 & 1 & 0 & 1 & 0 \\
0 & 0 & 0 & 0 & 0 & 0 & 1 \\
0 & 0 & 0 & 0 & 0 & 0 & 1 \\
0 & 1 & 1 & 1 & 1 & 0 & 0 \\
0 & 1 & 0 & 0 & 1 & 0 & 0 \\
1 & 0 & 0 & 0 & 0 & 0 & 0
\end{array}\right]
$$

Nós temos encontrados numéricamente que o número de órbitas periódicas de cada período até 17 preditas pelo shift de Markov são os mesmos que o número de órbitas periódicas encontradas pelo método de Biham-Wenzel.

Exemplo 6.2.2. Davis, Mackay and Sannami estudaram muitos valores dos parâmetros para transformações de Hénon que preservam área [DMS91]. Eles conjecturaram que $D_{3,3}$ define a frente de poda para $H_{5.65,1}$. Pelo algoritmo, a matriz de transição é:

$$
A_{2}=\left[\begin{array}{llllllllll}
1 & 0 & 1 & 0 & 0 & 0 & 0 & 0 & 0 & 0 \\
0 & 0 & 0 & 1 & 0 & 1 & 0 & 0 & 0 & 0 \\
0 & 0 & 0 & 0 & 1 & 1 & 0 & 0 & 0 & 0 \\
0 & 0 & 0 & 0 & 0 & 0 & 1 & 1 & 0 & 0 \\
0 & 0 & 0 & 0 & 0 & 0 & 1 & 0 & 1 & 0 \\
0 & 0 & 0 & 0 & 0 & 0 & 0 & 0 & 0 & 1 \\
0 & 0 & 0 & 0 & 0 & 0 & 0 & 0 & 0 & 1 \\
0 & 1 & 0 & 1 & 0 & 1 & 1 & 1 & 0 & 0 \\
0 & 1 & 0 & 1 & 0 & 0 & 0 & 1 & 0 & 0 \\
1 & 0 & 0 & 0 & 0 & 0 & 0 & 0 & 0 & 0
\end{array}\right]
$$




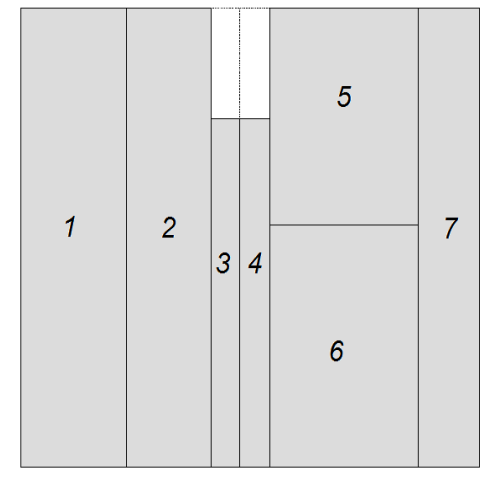

(a) Usando o disco de poda $D_{1,2}$ e...

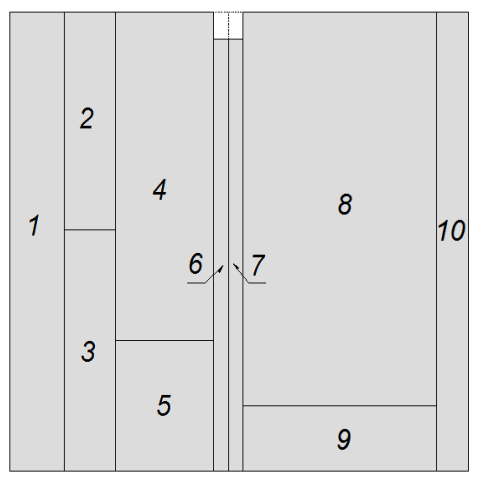

(b) usando o disco de poda $D_{3,3}$.

Figura 6.1: Partições de Markov conjecturadas para as transformações de Hénon $H_{3.5197,0.648}$ (figura 6.1(a)) e $H_{5.65,1}$ (figura 6.1(b)).

Esta matriz tem o mesmo número de órbitas periódicas de cada período (< 16) que o número de órbitas periódicas de $H_{5.65,1}$ encontradas pelo método de Biham-Wenzel (Compare com [DMS91]). Na figura 6.1(b) mostramos a partição de Markov associada.

Exemplo 6.2.3. Consideremos os valores $a=5.6917$. Numéricamente observamos que $H_{5.6917,1}$ pode ser considerado como um difeomorfismo de poda da ferradura usando o disco de poda $D_{4,4}$. A partição de Markov está na figura 6.2(a). A matriz associada é:

$$
A_{3}=\left[\begin{array}{llllllllllll}
1 & 1 & 0 & 0 & 0 & 0 & 0 & 0 & 0 & 0 & 0 & 0 \\
0 & 0 & 1 & 0 & 0 & 0 & 0 & 0 & 0 & 0 & 0 & 0 \\
0 & 0 & 0 & 1 & 1 & 0 & 0 & 0 & 0 & 0 & 0 & 0 \\
0 & 0 & 0 & 0 & 0 & 1 & 1 & 0 & 0 & 0 & 0 & 0 \\
0 & 0 & 0 & 0 & 0 & 0 & 0 & 1 & 0 & 0 & 0 & 0 \\
0 & 0 & 0 & 0 & 0 & 0 & 0 & 1 & 0 & 0 & 0 & 0 \\
0 & 0 & 0 & 0 & 0 & 0 & 0 & 0 & 1 & 1 & 1 & 1 \\
1 & 0 & 0 & 0 & 0 & 0 & 0 & 0 & 0 & 0 & 0 & 0 \\
0 & 0 & 0 & 0 & 1 & 1 & 0 & 0 & 1 & 1 & 1 & 1 \\
0 & 0 & 0 & 0 & 0 & 1 & 0 & 0 & 1 & 0 & 0 & 0 \\
0 & 0 & 0 & 0 & 1 & 0 & 0 & 0 & 0 & 1 & 0 & 0 \\
0 & 0 & 0 & 0 & 0 & 0 & 0 & 0 & 0 & 0 & 1 & 0
\end{array}\right]
$$

Também, esta matriz tem o mesmo número de órbitas periódicas que estas de $H_{5.6917,1}$ encontradas pelo método de Biham-Wenzel para períodos menores ou iguais que 14 .

\subsubsection{Usando dois discos de poda}

Exemplo 6.2.4. Outros valores estudados em [DMS91] são $(a, b)=(5.59,1)$. Para estos parâmetros, associamos dois discos de poda: $D_{2,3}$ e $D_{3,2}$. Nós 


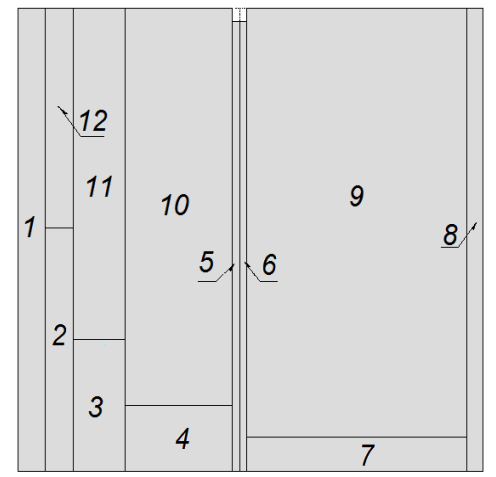

(a) Usando $D_{4,4}$ e $\ldots$

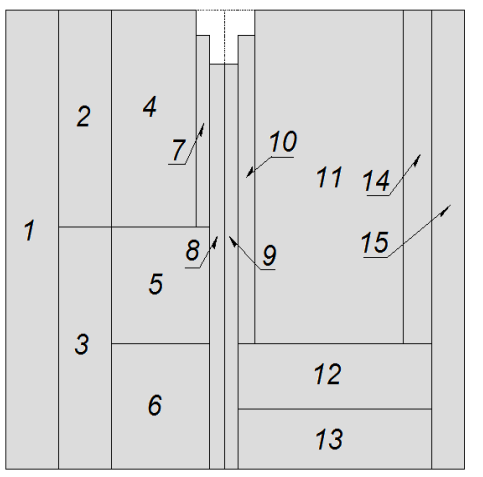

(b) .. usando dois discos de poda: $D_{2,3}$ e $D_{3,2}$.

Figura 6.2: Partições de Markov conjecturadas para $H_{5.6917,1}$ (figura 6.2(a)) e para $H_{5.59,1}$ (figura 6.2(b)).

construimos uma partição de Markov a qual está na figura 6.2(b) (Compare com [DMS91]). A matriz de transição é a seguinte:

$$
A_{4}=\left[\begin{array}{lllllllllllllll}
1 & 0 & 1 & 0 & 0 & 0 & 0 & 0 & 0 & 0 & 0 & 0 & 0 & 0 & 0 \\
0 & 0 & 0 & 0 & 1 & 0 & 0 & 1 & 0 & 0 & 0 & 0 & 0 & 0 & 0 \\
0 & 0 & 0 & 0 & 0 & 1 & 0 & 1 & 0 & 0 & 0 & 0 & 0 & 0 & 0 \\
0 & 0 & 0 & 0 & 0 & 0 & 0 & 0 & 1 & 1 & 1 & 0 & 0 & 0 & 0 \\
0 & 0 & 0 & 0 & 0 & 0 & 0 & 0 & 1 & 0 & 0 & 1 & 0 & 0 & 0 \\
0 & 0 & 0 & 0 & 0 & 0 & 0 & 0 & 1 & 0 & 0 & 0 & 1 & 0 & 0 \\
0 & 0 & 0 & 0 & 0 & 0 & 0 & 0 & 0 & 0 & 0 & 0 & 0 & 1 & 0 \\
0 & 0 & 0 & 0 & 0 & 0 & 0 & 0 & 0 & 0 & 0 & 0 & 0 & 0 & 1 \\
0 & 0 & 0 & 0 & 0 & 0 & 0 & 0 & 0 & 0 & 0 & 0 & 0 & 0 & 1 \\
0 & 0 & 0 & 0 & 0 & 0 & 0 & 0 & 0 & 0 & 0 & 0 & 0 & 1 & 0 \\
0 & 0 & 0 & 1 & 0 & 0 & 1 & 1 & 1 & 1 & 1 & 0 & 0 & 0 & 0 \\
0 & 1 & 0 & 1 & 0 & 0 & 1 & 0 & 0 & 1 & 1 & 0 & 0 & 1 & 0 \\
0 & 1 & 0 & 1 & 0 & 0 & 0 & 0 & 0 & 0 & 1 & 0 & 0 & 1 & 0 \\
0 & 1 & 0 & 0 & 0 & 0 & 0 & 0 & 0 & 0 & 0 & 0 & 0 & 0 & 0 \\
1 & 0 & 0 & 0 & 0 & 0 & 0 & 0 & 0 & 0 & 0 & 0 & 0 & 0 & 0
\end{array}\right]
$$

Exemplo 6.2.5. Considere os valores $(a, b)=(5.683,1)$. Numéricamente, associamos dois discos de poda: $D_{3,4}$ e $D_{4,3}$. A partição de Markov está na figura 
6.6. Assim a matriz de transição é:

$$
A_{5}=\left[\begin{array}{llllllllllllllllll}
1 & 1 & 0 & 0 & 0 & 0 & 0 & 0 & 0 & 0 & 0 & 0 & 0 & 0 & 0 & 0 & 0 & 0 \\
0 & 0 & 0 & 1 & 0 & 0 & 0 & 0 & 0 & 0 & 0 & 0 & 0 & 0 & 0 & 0 & 0 & 0 \\
0 & 0 & 0 & 0 & 1 & 0 & 1 & 0 & 0 & 0 & 0 & 0 & 0 & 0 & 0 & 0 & 0 & 0 \\
0 & 0 & 0 & 0 & 0 & 1 & 1 & 0 & 0 & 0 & 0 & 0 & 0 & 0 & 0 & 0 & 0 & 0 \\
0 & 0 & 0 & 0 & 0 & 0 & 0 & 1 & 1 & 0 & 0 & 0 & 0 & 0 & 0 & 0 & 0 & 0 \\
0 & 0 & 0 & 0 & 0 & 0 & 0 & 1 & 0 & 1 & 0 & 0 & 0 & 0 & 0 & 0 & 0 & 0 \\
0 & 0 & 0 & 0 & 0 & 0 & 0 & 0 & 0 & 0 & 1 & 0 & 0 & 0 & 0 & 0 & 0 & 0 \\
0 & 0 & 0 & 0 & 0 & 0 & 0 & 0 & 0 & 0 & 1 & 0 & 0 & 0 & 0 & 0 & 0 & 0 \\
0 & 0 & 0 & 0 & 0 & 0 & 0 & 0 & 0 & 0 & 0 & 1 & 1 & 1 & 1 & 1 & 1 & 1 \\
0 & 0 & 0 & 0 & 0 & 0 & 0 & 0 & 0 & 0 & 0 & 1 & 1 & 0 & 0 & 1 & 1 & 1 \\
1 & 0 & 0 & 0 & 0 & 0 & 0 & 0 & 0 & 0 & 0 & 0 & 0 & 0 & 0 & 0 & 0 & 0 \\
0 & 0 & 0 & 0 & 0 & 0 & 0 & 0 & 0 & 0 & 0 & 0 & 0 & 0 & 0 & 0 & 0 & 1 \\
0 & 0 & 0 & 0 & 0 & 0 & 1 & 1 & 0 & 0 & 0 & 0 & 1 & 1 & 1 & 1 & 1 & 0 \\
0 & 0 & 0 & 0 & 0 & 0 & 0 & 0 & 0 & 0 & 0 & 1 & 0 & 0 & 0 & 0 & 0 & 0 \\
0 & 0 & 0 & 0 & 0 & 0 & 0 & 0 & 0 & 0 & 0 & 1 & 0 & 0 & 0 & 0 & 0 & 0 \\
0 & 0 & 0 & 0 & 0 & 0 & 0 & 1 & 0 & 0 & 0 & 0 & 1 & 1 & 0 & 0 & 0 & 0 \\
0 & 0 & 0 & 0 & 0 & 0 & 1 & 0 & 0 & 0 & 0 & 0 & 0 & 0 & 1 & 1 & 0 & 0 \\
0 & 0 & 1 & 0 & 0 & 0 & 0 & 0 & 0 & 0 & 0 & 0 & 0 & 0 & 0 & 0 & 0 & 0
\end{array}\right]
$$

Esta matriz tem o mesmo número de órbitas periódicas que o número de órbitas período de $H_{a, b}$ encontradas pelo método de Biham-Wenzel para períodos menores que 16 .

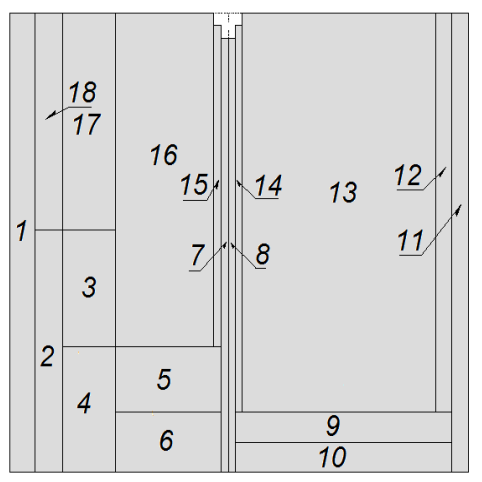

Figura 6.3: Partição de Markov usando os discos $D_{3,4}$ e $D_{4,3}$ conjecturada para $H_{5.683,1}$.

\subsubsection{Mais evidência numérica}

Exemplo 6.2.6. Considere a transformação de Hénon $H_{3.5,0.55}$. O disco de poda associado a esta transformação é $D_{2,3}$. A partição de Markov está na 
figura 6.4(a). A matriz de transição é:

$$
A_{6}=\left[\begin{array}{lllllllll}
1 & 1 & 0 & 0 & 0 & 0 & 0 & 0 & 0 \\
0 & 0 & 1 & 1 & 0 & 0 & 0 & 0 & 0 \\
0 & 0 & 0 & 0 & 1 & 1 & 0 & 0 & 0 \\
0 & 0 & 0 & 0 & 0 & 0 & 1 & 0 & 0 \\
0 & 0 & 0 & 0 & 0 & 0 & 1 & 0 & 0 \\
0 & 1 & 0 & 0 & 0 & 0 & 0 & 1 & 1 \\
1 & 0 & 0 & 0 & 0 & 0 & 0 & 0 & 0 \\
0 & 1 & 0 & 1 & 1 & 0 & 0 & 1 & 1 \\
0 & 0 & 0 & 0 & 1 & 0 & 0 & 1 & 0
\end{array}\right]
$$

Exemplo 6.2.7. Considere a transformação de Hénon $H_{2.766,0.4}$. Neste caso associamos dois discos de poda: $D_{0,3}$ e $D_{1,2}$. A partição de Markov está na figura 6.4(b). A matriz de transição é:

$$
A_{7}=\left[\begin{array}{lllllllllll}
1 & 1 & 0 & 0 & 0 & 0 & 0 & 0 & 0 & 0 & 0 \\
0 & 0 & 1 & 1 & 1 & 0 & 0 & 0 & 0 & 0 & 0 \\
0 & 0 & 0 & 0 & 0 & 1 & 1 & 1 & 0 & 0 & 0 \\
0 & 0 & 0 & 0 & 0 & 0 & 0 & 0 & 1 & 0 & 0 \\
0 & 0 & 0 & 0 & 0 & 0 & 0 & 0 & 0 & 1 & 0 \\
0 & 0 & 0 & 0 & 0 & 0 & 0 & 0 & 0 & 1 & 0 \\
0 & 0 & 0 & 0 & 0 & 0 & 0 & 0 & 1 & 0 & 0 \\
0 & 0 & 1 & 0 & 0 & 0 & 0 & 0 & 0 & 0 & 1 \\
0 & 1 & 0 & 0 & 0 & 0 & 0 & 0 & 0 & 0 & 0 \\
1 & 0 & 0 & 0 & 0 & 0 & 0 & 0 & 0 & 0 & 0 \\
0 & 0 & 1 & 1 & 0 & 0 & 1 & 0 & 0 & 0 & 1
\end{array}\right]
$$

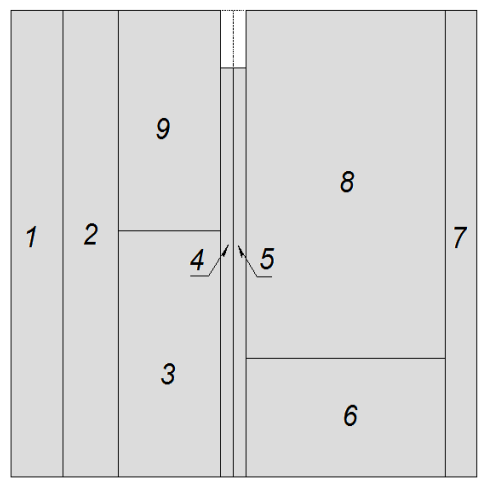

(a) Usando $D_{2,3}$ e...

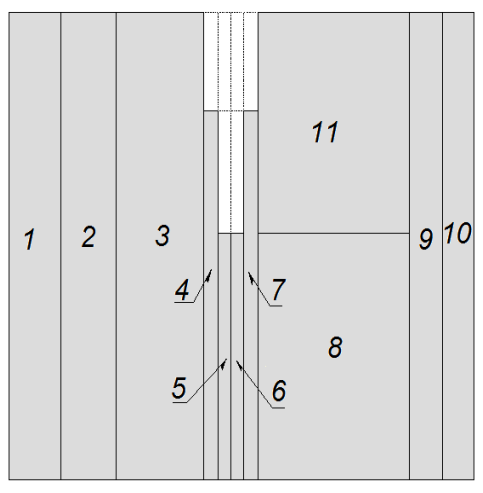

(b) .. usando dois discos de poda: $D_{0,3}$ e $D_{1,2}$.

Figura 6.4: Partições de Markov conjecturadas para $H_{3.5,0.55}$ (figura 6.4(a)) e para $H_{2.766,0.4}$ (figura 6.4(b)). 
Exemplo 6.2.8. Considere parâmetros próximos da transformação de Hénon $H_{2.887,0.4}$. Para esta transformação associamos o homeomorfismo de poda definido pelos discos $D_{1,3}$ e $D_{2,2}$. A partição de Markov está na figura 6.5(a) e a matriz de transição é:

$$
A_{8}=\left[\begin{array}{lllllllllllll}
1 & 1 & 0 & 0 & 0 & 0 & 0 & 0 & 0 & 0 & 0 & 0 & 0 \\
0 & 0 & 0 & 1 & 1 & 1 & 0 & 0 & 0 & 0 & 0 & 0 & 0 \\
0 & 0 & 0 & 0 & 0 & 0 & 1 & 1 & 0 & 0 & 0 & 0 & 0 \\
0 & 0 & 0 & 0 & 0 & 0 & 1 & 0 & 1 & 0 & 0 & 0 & 0 \\
0 & 0 & 0 & 0 & 0 & 0 & 0 & 0 & 0 & 1 & 0 & 0 & 0 \\
0 & 0 & 0 & 0 & 0 & 0 & 0 & 0 & 0 & 0 & 1 & 0 & 0 \\
0 & 0 & 0 & 0 & 0 & 0 & 0 & 0 & 0 & 0 & 1 & 0 & 0 \\
0 & 0 & 1 & 0 & 1 & 0 & 0 & 0 & 0 & 1 & 0 & 1 & 1 \\
0 & 0 & 1 & 0 & 0 & 0 & 0 & 0 & 0 & 1 & 0 & 1 & 0 \\
0 & 1 & 0 & 0 & 0 & 0 & 0 & 0 & 0 & 0 & 0 & 0 & 0 \\
1 & 0 & 0 & 0 & 0 & 0 & 0 & 0 & 0 & 0 & 0 & 0 & 0 \\
0 & 0 & 1 & 0 & 1 & 1 & 1 & 0 & 0 & 0 & 0 & 1 & 1 \\
0 & 0 & 0 & 0 & 0 & 0 & 0 & 0 & 0 & 1 & 0 & 0 & 0
\end{array}\right]
$$

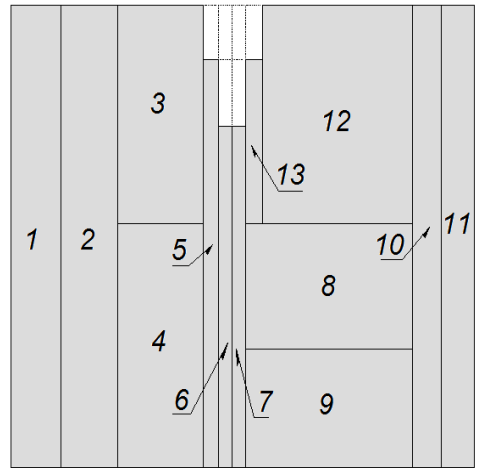

(a) Usando $D_{1,3}$ e $D_{2,2} \ldots$

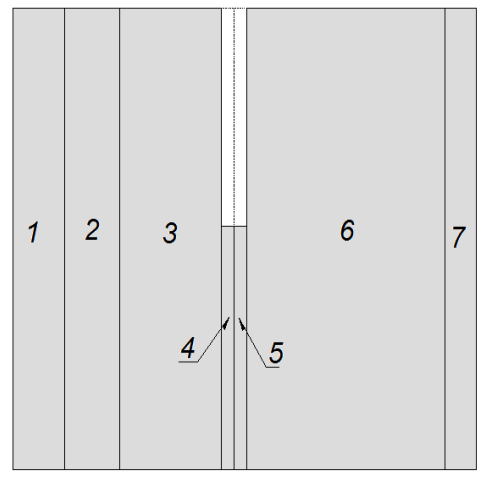

(b) .. usando o disco de poda: $D_{0,3}$.

Figura 6.5: Partições de Markov conjecturadas para $H_{2.887,0.4}$ (figura 6.5(a)) e para $H_{2.345,0.19}$ (figura 6.5(b)).

Exemplo 6.2.9. Considere os parâmetros $(a, b)=(2.345,0.19)$. Neste caso a transformação de Hénon $H_{a, b}$ é numericamente conjugada à transformação de poda utilizando o disco $D_{0,3}$. A partição de Markov está na figura $6.5(\mathrm{~b})$ e a matriz de transição é a seguinte:

$$
A_{9}=\left[\begin{array}{lllllll}
1 & 1 & 0 & 0 & 0 & 0 & 0 \\
0 & 0 & 1 & 1 & 0 & 0 & 0 \\
0 & 0 & 0 & 0 & 1 & 1 & 0 \\
0 & 0 & 0 & 0 & 0 & 0 & 1 \\
0 & 0 & 0 & 0 & 0 & 0 & 1 \\
0 & 1 & 1 & 0 & 0 & 1 & 0 \\
1 & 0 & 0 & 0 & 0 & 0 & 0
\end{array}\right]
$$


Exemplo 6.2.10. Considere os valores $(a, b)=(2.812,0.4)$. Os discos de poda conjecturados para esta transformação são $D_{1,2}$ e o disco de poda definido pelas órbitas homoclínicas $p_{0}=0^{\infty} 10111 \cdot 100110^{\infty}$ e $p_{1}=0^{\infty} 10110 \cdot 100110^{\infty}$. A partição de Markov está na figura 6.6 e a matriz de transição é a seguinte:

$$
A_{10}=\left[\begin{array}{llllllllllll}
1 & 1 & 0 & 0 & 0 & 0 & 0 & 0 & 0 & 0 & 0 & 0 \\
0 & 0 & 1 & 1 & 1 & 0 & 0 & 0 & 0 & 0 & 0 & 0 \\
0 & 0 & 0 & 0 & 0 & 1 & 1 & 0 & 0 & 0 & 0 & 0 \\
0 & 0 & 0 & 0 & 0 & 0 & 0 & 0 & 0 & 1 & 0 & 0 \\
0 & 0 & 0 & 0 & 0 & 0 & 0 & 0 & 0 & 0 & 1 & 0 \\
0 & 0 & 0 & 0 & 0 & 0 & 0 & 0 & 0 & 0 & 1 & 0 \\
0 & 0 & 1 & 0 & 0 & 0 & 0 & 0 & 0 & 0 & 0 & 1 \\
0 & 0 & 0 & 0 & 0 & 0 & 0 & 0 & 0 & 1 & 0 & 0 \\
0 & 0 & 1 & 1 & 0 & 0 & 0 & 1 & 1 & 0 & 0 & 0 \\
0 & 1 & 0 & 0 & 0 & 0 & 0 & 0 & 0 & 0 & 0 & 0 \\
1 & 0 & 0 & 0 & 0 & 0 & 0 & 0 & 0 & 0 & 0 & 0 \\
0 & 1 & 1 & 1 & 1 & 1 & 0 & 1 & 1 & 0 & 0 & 0
\end{array}\right]
$$

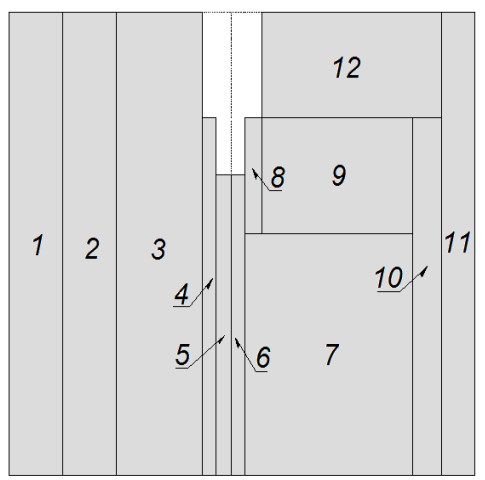

Figura 6.6: Partição de Markov conjecturada para $H_{2.812,0.4}$.

\subsection{Observações}

Na seção 5.2 provamos que, a menos de uma conjugação, existem transformações de Hénon que são difeomorfismos de poda. Pelo trabalho de Arai [Ara07b], eles correspondem a parâmetros que pertencem ao lugar real de ferraduras.

- Para $(a, b) \in[5.3125,5.46875] \times\{1\}$, associamos o disco de poda $D_{2,2}$; claramente, o automorfismo $\rho_{2,2}$ substitui as sequências 0010100 e 0011100 , uma pela outra, quando elas aparecem na codificação simbólica de um ponto de $\Sigma_{2}$.

- Para $(a, b) \in[2.21875,2.296875] \times\{0.25\}$, associamos o discos $D_{0,2}$. Neste caso vemos que o automorfismo do shift $\rho_{0,2}$ substitui as sequências 10100 e 11100 quando elas aparecem na codificação simbólica. 


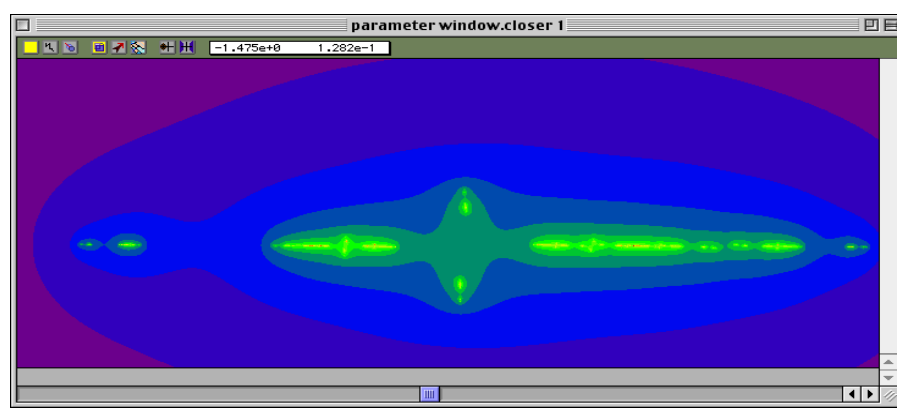

Figura 6.7: $(a \approx 2.8187+0.0119 i$ e $b=0.4)$.

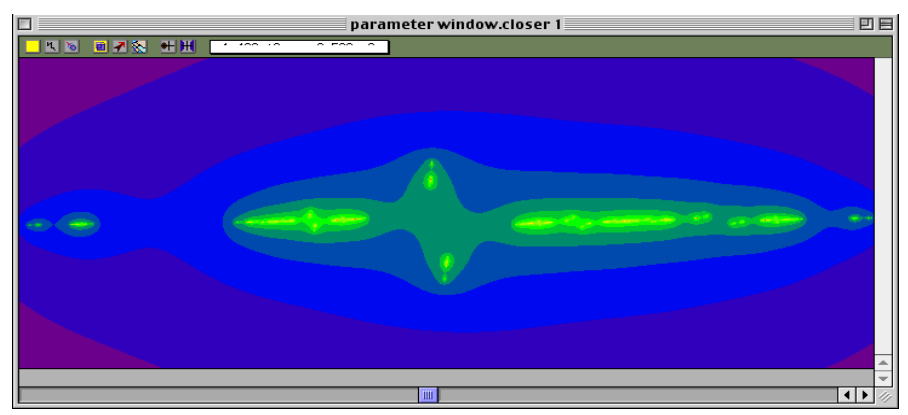

Figura 6.8: $(a \approx 2.8255-0.032 i$ e $b=0.4)$.

Também na seção 6.2, apresentamos evidência numérica indicando que outros parâmetros podem ser interpretados como difeomorfismos de poda. Nós conjecturamos que todos esses parâmetros estão no lugar de ferraduras, pois, ao parecer, existem caminhos em $\mathcal{H}^{\mathbb{C}}$ unindo estes parâmetros com um ponto em $D N$. Isto se deduz utilizando o programa SaddleDrop $^{1}$ e desenhando a variedade instável complexa do ponto fixo hiperbólico $0^{\infty}$.

Por exemplo para os valores $(2.812,0.4)$, pegamos 9 pontos no espaço de parâmetros complexos que aparentemente unem os valores $(2.812,0.4)$ com os valores $(3.149,0.4) \in D N$. Em todos os casos o conjunto de órbitas limitadas $K_{a, b}$, restrito à variedade instável complexa do ponto $0^{\infty}$, parece ser um conjunto de Cantor totalmente desconexo que varia continuamente quando os parâmetros são modificados. Veja desde a figura 6.7 até a figura 6.15. Isto sugere que todos os valores considerados estão no lugar de ferraduras.

Os automorfismos de poda conjecturados para alguns dos exemplos da seção 6.2 serão:

- Para alguma região ao redor de $(a, b)=(3.5197,0.648)$ associamos o disco de poda $D_{1,2}$. Acreditamos que $(a, b)$ está no lugar de ferraduras real e

\footnotetext{
${ }^{1}$ SaddleDrop é um programa criado por J. Hubbard e K. Papadantonakis para estudar a família de Hénon complexa. Este pode ser encontrado em http://www.math.cornell.edu/ dynamics/SD/index.html
} 


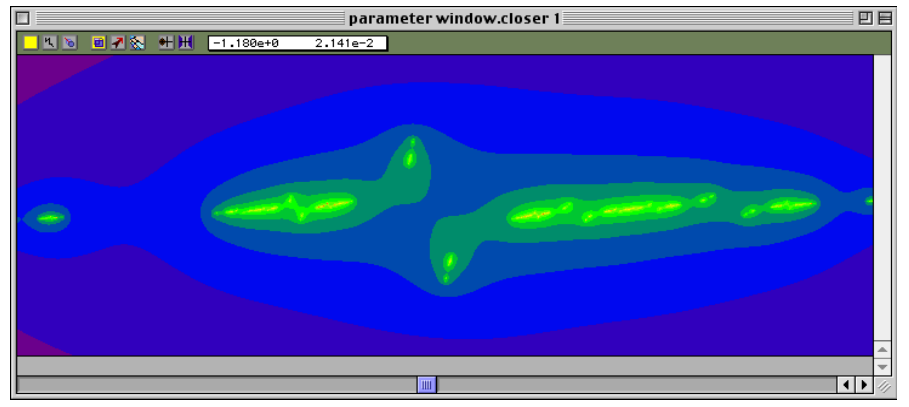

Figura 6.9: $(a \approx 2.8187-0.079 i$ e $b=0.4)$.

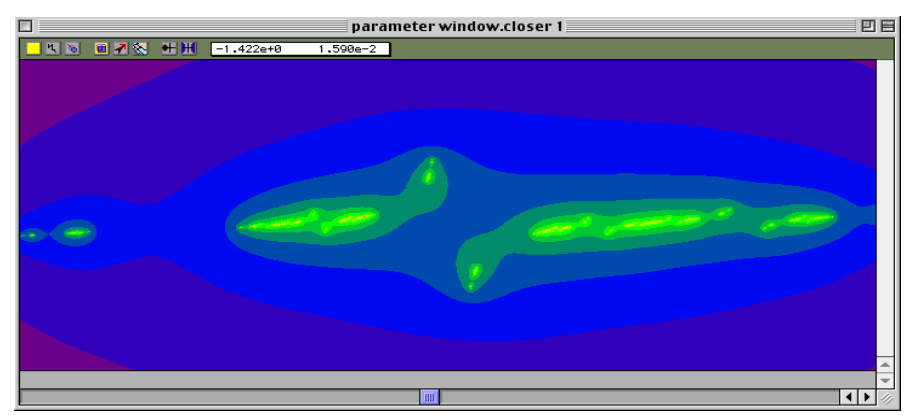

Figura 6.10: $(a \approx 2.8187-0.095 i$ e $b=0.4)$.

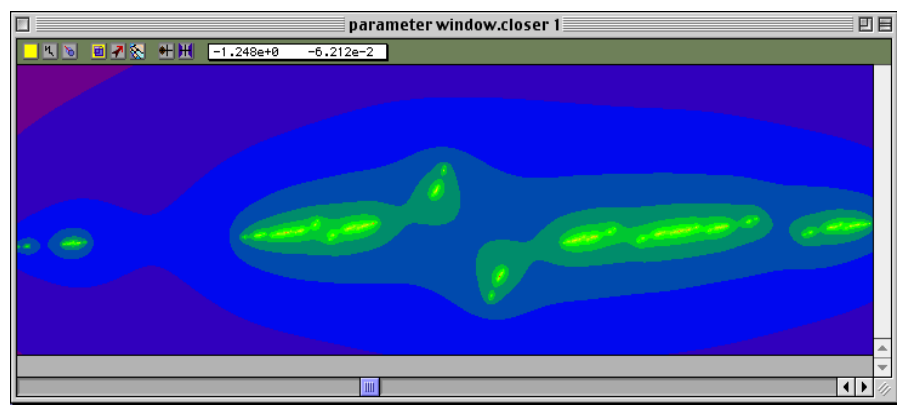

Figura 6.11: $(a \approx 2.8931-0.0959 i$ e $b=0.4)$. 


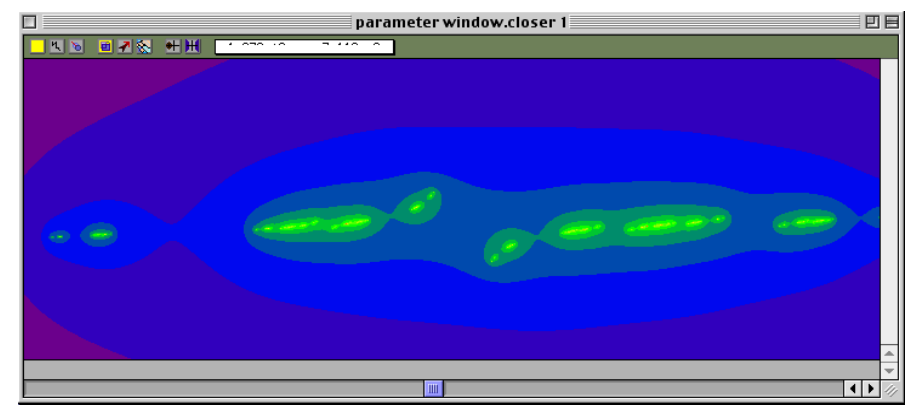

Figura 6.12: $(a \approx 3.00-0.0919 i$ e $b=0.4)$.

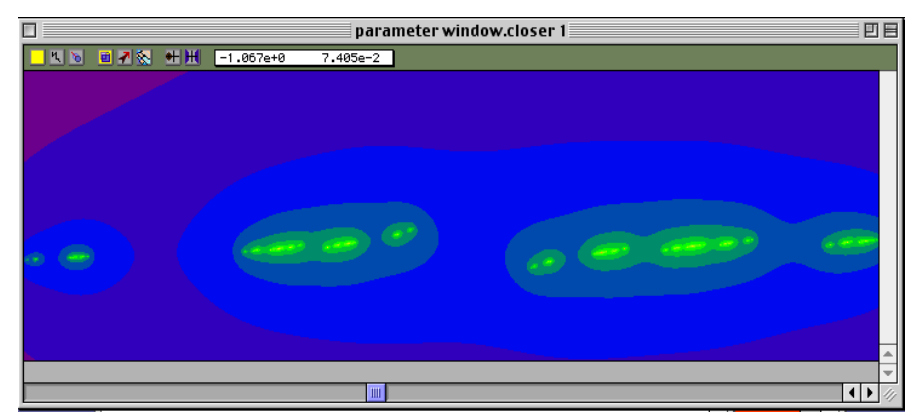

Figura 6.13: $(a \approx 3.1432-0.0919 i$ e $b=0.4)$.

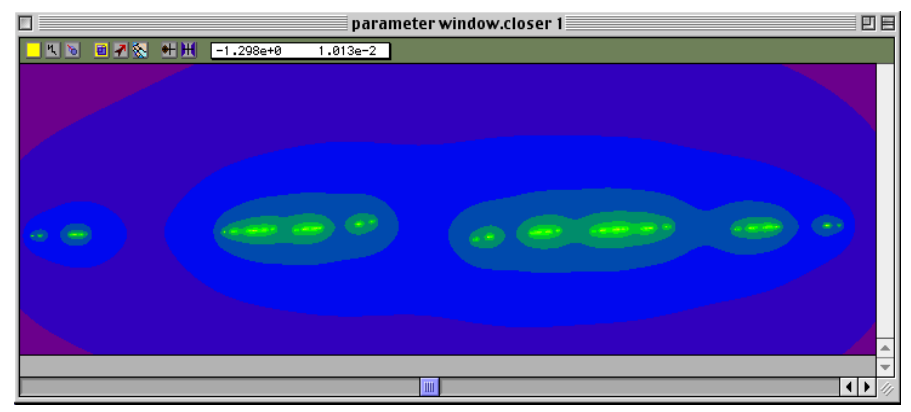

Figura 6.14: $(a \approx 3.1432-0.048 i$ e $b=0.4)$. 


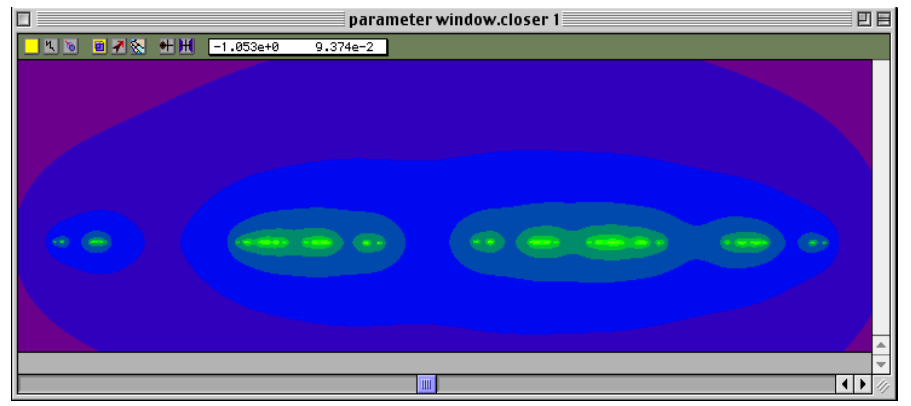

Figura 6.15: $(a \approx 3.149-0.00399 i$ e $b=0.4)$.

que o automorfismo do teorema 5.2.1 de Arai é o automorfismo de poda $\rho_{1,2}$ o qual substitui os blocos 010100 and 011100 .

- O mesmo pode ser dito para os valores estudados por Davis, Mackay e Sannami em [DMS91]. Para $(a, b)=(5.65,1)$ precisamos um disco de poda e para $(a, b)=(5.59,1)$ de dois discos de poda. No primeiro caso, o disco é $D_{3,3}$ e o automorfismo substitui as sequências 000101000 e 000111000 ; no segundo caso associamos os discos $D_{2,3}$ e $D_{3,2}$ e o automorfismo substitui as sequências 100101000 e 100111000 , una pela outra, e as sequências 00010100 e 00011100 uma pela outra.

- Considere os valores $(a, b)=(5.683,1)$. Neste caso associamos dois discos de poda: $D_{3,4}$ e $D_{4,3}$. Para os valores $(a, b)=(5.6917,1)$, associamos o disco de poda $D_{4,4}$. Nestes casos é facil ver quais são os automorfismos de poda correspondentes.

\subsection{Conjectura da Frente de poda no lugar de Ferraduras}

Gostariamos de ressaltar a similaridade entre o teorema 5.2.1 de Arai e o teorema 5.2.2. Esta semelhança diz que existe um ponto de contacto entre um difeomorfismo de poda e uma transformação de Hénon que está no lugar de ferraduras real. Este ponto de contacto é o automorfismo do shift. Também, numericamente, observamos que há laços não triviais no lugar de ferraduras que passam por transformações de Hénon cujos automorfismos de monodromia são automorfismos de poda. Conjecturamos que isto também é verdade para cada laço que passa pelo lugar real de ferraduras e que começa em $D N$. Portanto, uma poda com discos homoclínicos pode ser interpretada como a ação de um elemento de $\operatorname{Aut}\left(\Sigma_{2}\right)$ o qual fixa algum subconjunto de $\Sigma_{2}$. Assim uma transformação $H_{a, b}$ com $(a, b) \in \mathcal{H}_{0}^{\mathbb{C}} \cap \mathbb{R}^{2}$ poderia ser considerada como um difeomorfismo de poda e o automorfismo do shift associado como um automorfismo de poda. No caso que $H_{a, b}$ preserva área, pela seção 6.1, estes automorfismos de monodromia deveriam ter uma forma particular: Eles provêm de frentes de poda simétricos.

Pelo Teorema 1.3.3, se uma transformação de Hénon $H_{a, b}$ tem entropia maximal mas não é hiperbólica, existem difeomorfismos de poda hiperbólicos arbi- 
trariamente próximos a ela. Assim, pensamos que as afirmações anteriores são verdadeiras, em particular, para muitos parâmetros próximos a estas transformações.

Acreditamos que a caracterização topologica do conjunto não errante real $\Omega(H) \cap \mathbb{R}^{2}$ de uma transformação de Hénon que preserva orientação e que está no lugar real de ferraduras, é dada pela dinâmica simbólica de um difeomorfismo de poda como no Teorema 1.3.1. Assim, reformulamos a CFP em termos de discos homoclínicos:

Conjectura: Dada uma transformação de Hénon real $H_{a, b}$ com $(a, b) \in$ $\mathcal{H}_{0}^{\mathbb{C}} \cap \mathbb{R}^{2}$ e $b>0$, existe uma coleção $\mathcal{D}$ de discos de poda homoclínicos e um laço $\gamma \in \mathcal{H}^{\mathbb{C}}$ que passa pelo ponto $(a, b)$ e que começa em $D N$ tais que o automorfismo de monodromia associado a $\gamma$ é o automorfismo de poda associado à coleção $\mathcal{D}$. 


\section{Apêndice A}

\section{O metodo de Biham-Wenzel}

Em [BW89], Ofer Biham e Wolfgang Wenzel introduziram um método para encontrar órbitas periódicas. Embora o metodo carece de um fundamento matemático que garante a convergencia a uma solução, tem sido muito utilizado para descrever numéricamente a natureza do conjunto não errante de transformações do plano. Nós temos utilizado ele para encontrar órbitas periódicas da transformação de Hénon para valores $a$ e $b$ dados.

Podemos descrever o metodo da seguinte maneira. Suponha que temos um sistema da forma:

$$
\left\{\begin{array}{c}
x_{n+1}=F\left(x_{n}, y_{n}\right) \\
y_{n+1}=x_{n}
\end{array}\right.
$$

Ou seja, $x_{n+1}=F\left(x_{n}, x_{n-1}\right)$. Estamos interessados em encontrar uma órbita $N$-periódica: $p_{1}, p_{2}, \ldots, p_{N}$, com $p_{0}:=p_{N}, p_{N+1}:=p_{1}$. Suponha que esta órbita periódica tem um codificação simbólica $\overline{s_{1} \ldots s_{N}}$, então defina a Codificação Biham-Wenzel por $\overline{S_{1} \ldots S_{N}}$ com:

$$
S_{i}:=\left\{\begin{array}{cc}
1 & \text { se } s_{i}=0 \\
-1 & \text { se } s_{i}=1
\end{array}\right.
$$

Em outras palavras, no caso da família de Hénon, a codificação Biham-Wenzel diz, supondo que $p_{i} \neq 0, \forall i \in\{1, \ldots, N\}$ :

$$
S_{i}:=\left\{\begin{array}{cc}
1 & \text { se } p_{i}<0 \\
-1 & \text { se } p_{i}>0
\end{array}\right.
$$

Isto é $S_{i} p_{i}<0, \forall i=1, . ., N$. Considere o sistema $n$-dimensional [GKM89]:

$$
\frac{d x_{i}}{d t}=S_{i}\left(x_{i+1}-F\left(x_{i}, x_{i-1}\right)\right),
$$

para todo $i \in\{1, \ldots, N\}$ com $x_{0}:=x_{N}$ e $x_{N+1}:=x_{1}$. Observe que uma solução estacionaria do sistema de equações diferenciáveis A.4 corresponde a uma órbita periódica de A.1 e viceversa. Assim, o problema de encontrar a órbita $\mathrm{N}$ periódica se reduz a encontrar um ponto de equilibrio do sistema A.4 o que pode ser feito utilizando o método de Runge-Kutta. 
No caso da família de Hénon, $F\left(x_{n}, x_{n-1}\right)=a-x_{n}^{2}-b x_{n-1}$, existe um teorema que, sob certas condições na órbita e o parâmetro $b$, garanta a convergência a um ponto de equilibrio.

Teorema A.0.1. Suponha que $\mathrm{p}=\left\{p_{1}, \ldots ., p_{N}\right\}$ é uma órbita periódica de A.1 e suponha que existe um $\epsilon>0$ talque $p_{i} S_{i} \leq-\epsilon, \forall i$ e $\epsilon>\frac{1+b}{2}$ então $\mathrm{p}$ é um ponto crítico atrator do sistema A.4.

Demonstração. Pelas hipoteses: $2 S_{i} p_{i}+\left|S_{i}\right|+\left|b S_{i}\right| \leq-2 \epsilon+1+b<0$. Logo $\left|S_{i}\right|+\left|b S_{i}\right|<\left|2 S_{i} p_{i}\right|$. A derivada do campo é:

$$
D F(p)=\left[\begin{array}{cccccccc}
2 S_{1} p_{1} & S_{1} & 0 & . & . . & . . & 0 & b S_{1} \\
b S_{2} & 2 S_{2} p_{2} & S_{2} & 0 & . . & . . & . . & 0 \\
0 & b S_{3} & 2 S_{3} p_{3} & S_{3} & 0 & . . & . . & 0 \\
\vdots & \vdots & \vdots & \vdots & \vdots & \vdots & \vdots & \vdots \\
0 & . . & . . & . . & 0 & b S_{N-1} & 2 S_{N-1} p_{N-1} & S_{N-1} \\
S_{N} & 0 & . . & . . & . . & 0 & b S_{N} & 2 S_{N} p_{N}
\end{array}\right]
$$

Seja $\lambda$ um autovalor de $D F(p)$, então, pelo teorema de Gershgorin:

$$
\left|\lambda-2 S_{i} p_{i}\right| \leq\left|S_{i}\right|+\left|b S_{i}\right|<\left|2 S_{i} p_{i}\right|
$$

Dado que $2 S_{i} p_{i}$ é negativo, temos que $\operatorname{Re}(\lambda)<0$.

Para outros parâmetros simplesmente utilizamos o metodo de Runge-Kutta e se vermos que este parece convergir então consideramos que temos encontrado a órbita periódica procurada. Se o método parece divergir então dizemos que não existe sequência periódica com a codificação Biham-Wenzel dada. 


\section{Referências Bibliográficas}

[Ara07a] Zin Arai. On hyperbolic plateaus of the Hénon map. Experiment. Math., 16(2):181-188, 2007.

[Ara07b] Zin Arai. On loops in the hyperbolic locus of the complex Hénon map and their monodromies. arXiv:math.DS/0704.2978v1, 2007.

[BC91] Michael Benedicks and Lennart Carleson. The dynamics of the Hénon map. Ann. of Math. (2), 133(1):73-169, 1991.

[BLS93] Eric Bedford, Mikhail Lyubich, and John Smillie. Polynomial diffeomorphisms of $\mathbf{C}^{2}$. IV. The measure of maximal entropy and laminar currents. Invent. Math., 112(1):77-125, 1993.

[BS91] Eric Bedford and John Smillie. Polynomial diffeomorphisms of $\mathbf{C}^{2}$ : currents, equilibrium measure and hyperbolicity. Invent. Math., 103(1):69-99, 1991.

[BS04] Eric Bedford and John Smillie. Real polynomial diffeomorphisms with maximal entropy: Tangencies. Ann. of Math. (2), 160(1):1$26,2004$.

[BS06a] Eric Bedford and John Smillie. The Hénon family: the complex horseshoe locus and real parameter space. In Complex dynamics, volume 396 of Contemp. Math., pages 21-36. Amer. Math. Soc., Providence, RI, 2006.

[BS06b] Eric Bedford and John Smillie. Real polynomial diffeomorphisms with maximal entropy. II. Small Jacobian. Ergodic Theory Dynam. Systems, 26(5):1259-1283, 2006.

[BW89] Ofer Biham and Wolfgang Wenzel. Characterization of unstable periodic orbits in chaotic attractors and repellers. Phys. Rev. Lett., 63(8):819-822, 1989.

[CGP88] Predrag Cvitanović, Gemunu H. Gunaratne, and Itamar Procaccia. Topological and metric properties of Hénon-type strange attractors. Phys. Rev. A (3), 38(3):1503-1520, 1988. 
[Cvi91] Predrag Cvitanović. Periodic orbits as the skeleton of classical and quantum chaos. Phys. D, 51(1-3):138-151, 1991. Nonlinear science: the next decade (Los Alamos, NM, 1990).

[dC99] André de Carvalho. Pruning fronts and the formation of horseshoes. Ergodic Theory Dynam. Systems, 19(4):851-894, 1999.

[dCH01] André de Carvalho and Toby Hall. Pruning theory and Thurston's classification of surface homeomorphisms. J. Eur. Math. Soc. (JEMS), 3(4):287-333, 2001.

[dCH02a] André de Carvalho and Toby Hall. The forcing relation for horseshoe braid types. Experiment. Math., 11(2):271-288, 2002.

[dCH02b] André de Carvalho and Toby Hall. How to prune a horseshoe. Nonlinearity, 15(3):R19-R68, 2002.

[dCH10] André de Carvalho and Toby Hall. Decoration invariants for horseshoe braids. Discrete Contin. Dyn. Syst., 27(3):863-906, 2010.

[Dev89] Robert L. Devaney. An introduction to chaotic dynamical systems. Addison-Wesley Studies in Nonlinearity. Addison-Wesley Publishing Company Advanced Book Program, Redwood City, CA, second edition, 1989.

[Dil] James Dillon. Trabalho não publicado.

[DMS91] M. J. Davis, R. S. MacKay, and A. Sannami. Markov shifts in the Hénon family. Phys. D, 52(2-3):171-178, 1991.

[DN79] R. Devaney and Z. Nitecki. Shift automorphisms in the Hénon mapping. Comm. Math. Phys., 67(2):137-146, 1979.

[Fro10] R. Frongillo. Topological entropy bounds for hyperbolic plateaus of the Hénon map. arXiv:math.DS/1001.4211v1, 2010.

[FS92] John Erik Fornæss and Nessim Sibony. Complex Hénon mappings in $\mathbf{C}^{2}$ and Fatou-Bieberbach domains. Duke Math. J., 65(2):345-380, 1992.

[GKM89] P. Grassberger, H. Kantz, and U. Moenig. On the symbolic dynamics of the Hénon map. J. Phys. A, 22(24):5217-5230, 1989.

[HC98] K. Hansen and P. Cvitanović. Bifurcation structures in maps of Hénon type. Nonlinearity, 11(5):1233-1261, 1998.

[Hén76] M. Hénon. A two-dimensional mapping with a strange attractor. Comm. Math. Phys., 50(1):69-77, 1976.

[Hoe08] Ulrich A. Hoensch. Some hyperbolicity results for Hénon-like diffeomorphisms. Nonlinearity, 21(3):587-611, 2008. 
[HOV94] John H. Hubbard and Ralph W. Oberste-Vorth. Hénon mappings in the complex domain. I. The global topology of dynamical space. Inst. Hautes Études Sci. Publ. Math., (79):5-46, 1994.

[HOV95] John H. Hubbard and Ralph W. Oberste-Vorth. Hénon mappings in the complex domain. II. Projective and inductive limits of polynomials. In Real and complex dynamical systems (Hillerød, 1993), volume 464 of NATO Adv. Sci. Inst. Ser. C Math. Phys. Sci., pages 89-132. Kluwer Acad. Publ., Dordrecht, 1995.

[HS04] R. Hagiwara and A. Shudo. An algorithm to prune the areapreserving Hénon map. J. Phys. A, 37(44):10521-10543, 2004.

[KH95] Anatole Katok and Boris Hasselblatt. Introduction to the modern theory of dynamical systems, volume 54 of Encyclopedia of Mathematics and its Applications. Cambridge University Press, Cambridge, 1995. With a supplementary chapter by Katok and Leonardo Mendoza.

[MNTU00] S. Morosawa, Y. Nishimura, M. Taniguchi, and T. Ueda. Holomorphic dynamics, volume 66 of Cambridge Studies in Advanced Mathematics. Cambridge University Press, Cambridge, 2000. Translated from the 1995 Japanese original and revised by the authors.

[MT88] J. Milnor and W. Thurston. On iterated maps of the interval. In Dynamical systems (College Park, MD, 1986-87), volume 1342 of Lecture Notes in Math., pages 465-563. Springer, Berlin, 1988.

[New79] Sheldon E. Newhouse. The abundance of wild hyperbolic sets and nonsmooth stable sets for diffeomorphisms. Inst. Hautes Études Sci. Publ. Math., (50):101-151, 1979.

[New04] Sheldon Newhouse. Cone-fields, domination, and hyperbolicity. In Modern dynamical systems and applications, pages 419-432. Cambridge Univ. Press, Cambridge, 2004.

[OV87] R. Oberste-Vorth. Complex horseshoes and the dynamics of mappings of two complex variables. PhD thesis, Cornell University, 1987.

[PT93] Jacob Palis and Floris Takens. Hyperbolicity and sensitive chaotic dynamics at homoclinic bifurcations, volume 35 of Cambridge Studies in Advanced Mathematics. Cambridge University Press, Cambridge, 1993. Fractal dimensions and infinitely many attractors.

[Shu87] Michael Shub. Global stability of dynamical systems. SpringerVerlag, New York, 1987. With the collaboration of Albert Fathi and Rémi Langevin, Translated from the French by Joseph Christy. 
[Sma67] S. Smale. Differentiable dynamical systems. Bull. Amer. Math. Soc., 73:747-817, 1967. 